\title{
DEVELOPMENT OF A MESHLESS METHOD TO SOLVE COMPRESSIBLE POTENTIAL FLOWS
}

\author{
A Thesis \\ presented to \\ the Faculty of California Polytechnic State University, \\ San Luis Obispo
}

\author{
In Partial Fulfillment \\ of the Requirements for the Degree \\ Master of Science in Aerospace Engineering
}

by

Alejandro Ramos

June 2010 
(C) 2010

Alejandro Ramos

ALL RIGHTS RESERVED 


\title{
COMMITTEE MEMBERSHIP
}

\author{
TITLE : $\quad$ DEVELOPMENT OF A MESHLESS METHOD TO SOLVE \\ COMPRESSIBLE POTENTIAL FLOWS
}

\begin{abstract}
AUTHOR: Alejandro Ramos
\end{abstract}
DATE SUBMITED: June 2010

Dr. Rob A. McDonald

Advisor and Committee Chair

Aerospace Engineering

Dr. David D. Marshall

Committee Member

Aerospace Engineering

Dr. Kim A. Shollenberger

Committee Member

Mechanical Engineering

Dr. Timothy Takahashi

Committee Member

Northrop Grumman Corporation 


\begin{abstract}
Development of a Meshless Method to Solve Compressible Potential Flows

Alejandro Ramos
\end{abstract}

The utility of computational fluid dynamics (CFD) for solving problems of engineering interest has experienced rapid growth due to the improvements in both memory capacity and processing speed of computers. While the capability now exists for the solution of the Navier-Stokes equations about complex and complete aircraft configurations, the bottleneck within the process is the time consuming task of properly generating a mesh that can accurately solve the governing partial differential equations (PDEs). This thesis explored two numerical techniques that attempt to circumvent the difficulty associated with the meshing process by solving a simplified form of the continuity equation within a meshless framework. The continuity equation reduces to the full potential equation by assuming irrotational flow. It is a nonlinear PDE that can describe flows for a wide spectrum of Mach numbers that do not exhibit discontinuities. It may not be an adequate model for the detailed analysis of a complex flowfield since viscous effects are not captured by this equation, but it is an appealing alternative for the aircraft designer because it can provide a quick and simple to implement estimate of the aerodynamic characteristics during the conceptual design phase.

The two meshless methods explored in this thesis are the Dual Reciprocity Method (DRM) and the Generalized Finite Difference Method (GFD). The Dual Reciprocity Method was shown to have the capability to solve for the two-dimensional subcritical compressible $\left(M_{\infty}=0.375\right)$ flow over a Circular Cylinder and the non-lifting flow $\left(\alpha=0^{\circ}, M_{\infty}=0.72\right)$ for a NACA 0012 airfoil. Unfortunately these solutions were obtained with the requirement of a priori knowledge of the solution to tune a parameter necessary for proper convergence of the algorithm. Due to the shortcomings of applying the Dual Reciprocity Method, the Generalized Finite Difference Method was also investigated. The GFD method solves a PDE in differential form and can be thought of as a meshless form of a standard finite difference scheme. This method proved to be an accurate and general technique for solving the previously mentioned cases along with the lifting flow $\left(\alpha=2^{\circ}, M_{\infty}=0.63\right)$ about a NACA 0012 airfoil. It was also demonstrated that the GFD method could be formulated to discretize the full potential equation with second order accuracy. Both solution methods offer their own set of unique advantages and challenges, but it was determined that the GFD Method possessed the flexibility necessary for a meshless technique to become a viable aerodynamic design tool. 


\section{ACKNOWLEDGMENTS}

There are many people that I am indebted to for all the support they have provided me during the past six years. The help and encouragement they have given me has made the research presented in this thesis possible.

I would first like to recognize the contributions my advisor Dr. McDonald has made in shaping this research and the mentoring he has provided over the past few years. When we first met in my aircraft design class over two years ago, I remember approaching him one evening about potential thesis research topics. The enthusiasm and passion that he showed while explaining the, confusing at the time, Dual Reciprocity Method is a prime example of how he approaches his job with excitement and has the ability to transfer those feelings to his students. It truly has been a pleasure to work with him and I am grateful for all the advice he has given me. I would also like to thank Craig Nickol, the primary technical monitor for the NASA Research Announcement Grant NNX07AO14A, which supported this work.

I also have to acknowledge all the friends I have made during my time at Cal Poly. The tight knit group of Aero friends I have made over the years provided a competitive yet fun environment to work in. I want to especially recognize all the guys in the computer lab, past and present. The amount of work we have done slaving away in front of computer screens can only be exceeded by the amount of fun we had doing it. To the present members Jake, Vallone, and all the knuckleheads on Marshall's side of the room: the past couple of years have been a blast and the lab would have been a boring and sad place without you. I am going to miss all the ridiculous conversations that have taken place in that room and I wish you all the best.

Lastly, I want to express my feelings of love and gratitude for my family. This thesis would not have been possible without their support and patience. The weekends when I had the opportunity to come home afforded me with a much needed break from my studies and not to mention the awesome home cooked meals. I truly cherished all the opportunities I had to hang out with my sister Veronica and all my cousins. The times we had together reminded me how important it is for family to stick together. My parents Sergio and Lilia have worked hard to provide the opportunities to reach my full potential and I hope that this thesis is the realization of not only my hard work but also theirs. I want to also thank my grandparents Enrique and Raquel for all the prayers they have said for me over the past few weeks. You are truly the pillars of our family and I can't thank you enough for all you have done for me. I love you all and I hope I have made you proud. 


\section{Table of Contents}

List of Tables $\quad$ ix

List of Figures $\quad$ xii

Nomenclature $\quad$ xiii

1 Introduction $\quad 1$

1.1 Motivation . . . . . . . . . . . . . . . . . . . . 1

1.2 Scope of the Present Thesis . . . . . . . . . . . . . . . 4

2 Potential Aerodynamics 6

2.1 The Full Potential Equation . . . . . . . . . . . . . . . . 6

2.2 Laplace's Equation . . . . . . . . . . . . . . . . . . . . 11

3 Panel Methods $\quad 13$

3.1 The Boundary Integral Equation . . . . . . . . . . . . . . 15

3.1.1 Mathematical Formulation . . . . . . . . . . . . . . . 15

3.1 .2 Numerical Implementation . . . . . . . . . . . . . . . . . . . 21

3.2 Code Verification . . . . . . . . . . . . . . . . . . . . . . . 27

3.2.1 Circular Cylinder . . . . . . . . . . . . . . . . . . . . 28

3.2.2 Lifting Airfoil . . . . . . . . . . . . . . . 30

4 The Dual Reciprocity Method 33

4.1 Formulation . . . . . . . . . . . . . . . . . . 34

4.1.1 Simple Poisson Term . . . . . . . . . . . . . . . 35

4.1 .2 Complex RHS . . . . . . . . . . . . . . . . . . . 41

4.2 Formulation for Compressible Flows . . . . . . . . . . . . . . . . 46

4.2.1 DRM Solution Procedure. . . . . . . . . . . . . . 47

4.2.2 RBF Selection Criteria .............. 50

4.3 Results ........................ 53

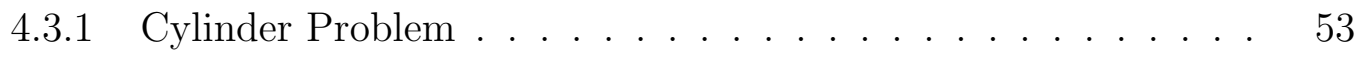

4.3.2 Airfoil Problem . . . . . . . . . . . . . . . 60 
5 The Generalized Finite Difference Method 64

5.1 Solution Algorithm . . . . . . . . . . . . . . . . . . . 68

5.1 .1 Cloud Generation . . . . . . . . . . . . . . . . 68

5.1.2 Derivative Coefficient Calculation . . . . . . . . . . . . 70

5.1 .3 Solution Initialization . . . . . . . . . . . . . . . . . . 73

5.1 .4 Solution Update . . . . . . . . . . . . . . . . . . . . . . 74

5.1.5 Convergence Estimation _. . . . . . . . . . . . . . . 77

5.2 Implementation Considerations . . . . . . . . . . . . . . 77

5.2.1 Neumann Boundary Condition Enforcement . . . . . . . . 78

5.2 .2 Trailing Edge Treatment . . . . . . . . . . . . . . . . . . . 85

5.2 .3 Wake Treatment . . . . . . . . . . . . . . . . 87

5.3 Order of Accuracy Estimates . . . . . . . . . . . . . . . . . . . . 89

5.3.1 In Domain Discretization Error . . . . . . . . . . . . . . 91

5.3.2 On Boundary Discretization Error . . . . . . . . . . . . . 94

6 Genralized Finite Difference Method Results 97

6.1 Circular Cylinder . . . . . . . . . . . . . . . . . . . . . . . 97

6.1 .1 Convergence Criteria . . . . . . . . . . . . . . . . . . . 101

6.1.2 Number of Support Points . . . . . . . . . . . . . . . . . 102

6.1.3 Influence of the Farfield Boundary . . . . . . . . . . . . . . . 104

6.1 .4 Number of Boundary Nodes . . . . . . . . . . . . . . . . 106

6.1.5 Growth Ratio . . . . . . . . . . . . . . . . . . . . . . . 107

6.1.6 Variation with $M_{\infty} \ldots \ldots \ldots \ldots \ldots \ldots$

$6.2 \quad$ Non-Lifting NACA $0012 \ldots \ldots \ldots$

6.2.1 Convergence Criteria . . . . . . . . . . . . . . . . 115

6.2 .2 Number of Support Points . . . . . . . . . . . . . . . . . 118

6.2 .3 Influence of the Farfield Boundary . . . . . . . . . . . . . . 120

6.2.4 Number of Boundary Points . . . . . . . . . . . . . . . . . 121

6.2 .5 Growth Ratio . . . . . . . . . . . . . . . . . . . . . 123

6.3 Lifting NACA $0012 \ldots \ldots \ldots \ldots \ldots \ldots$

$\begin{array}{lll}7 & \text { Final Remarks } & 129\end{array}$

7.1 Unique Contributions . . . . . . . . . . . . . . . . . . . . . . . . . . 129

7.2 Comparison of the Two Methods . . . . . . . . . . . . . 131

7.3 Future Work . . . . . . . . . . . . . . . . . . . . . . . . 134

$\begin{array}{ll}\text { References } & 138\end{array}$

$\begin{array}{ll}\text { Appendices } & 150\end{array}$ 
$\begin{array}{ll}\text { A Influence Coefficients } & 150\end{array}$

A.1 $[H]$ Matrix . . . . . . . . . . . . . . . . . . . . . . 150

A.2 $[G]$ Matrix . . . . . . . . . . . . . . . . . . . . . . . . . . 152

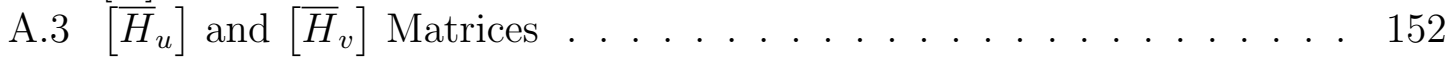

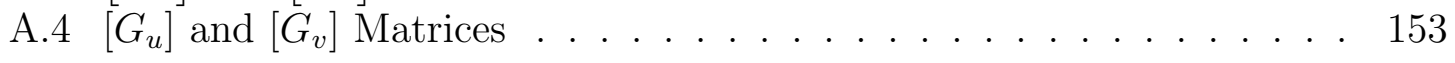




\section{List of Tables}

4.1 Domain Node Results . . . . . . . . . . . . . . . . . . . . . . 46

6.1 Baseline Distribution Metrics . . . . . . . . . . . . . . . . 99

6.2 Suction Peak Error Due to the Amount of Cloud Support Points . . . 104

6.3 Cylinder Mesh Refinement Data . . . . . . . . . . . . . . . . . 107

6.4 Cylinder Growth Ratio Study Data . . . . . . . . . . . . . . 108

6.5 NACA 0012 Baseline Distribution Metrics . . . . . . . . . . . . 113

6.6 Lift Coefficient as a Function of the Number of Support Points . . . . 120

6.7 Lift Coefficient as a function of the Farfield Radius . . . . . . . . 120

6.8 Mean Distances for Clouds Associated with the Leading Edge Point . 123

6.9 Airfoil Ratio Study Data . . . . . . . . . . . . . . . . . . . . . 124 


\section{List of Figures}

2.1 Problem Domain . . . . . . . . . . . . . . . . . . . . . . . 10

3.1 Integration Paths $\ldots \ldots \ldots \ldots \ldots \ldots \ldots \ldots \ldots$

3.2 Surface Discretization . . . . . . . . . . . . . . . . . . . . . . . . 22

3.3 Coupled System Setup . . . . . . . . . . . . . . . . . . . . 27

3.4 Circular Cylinder Geometry . . . . . . . . . . . . . . . . . 28

3.5 Circular Cylinder $C_{p}$ Distribution $\ldots \ldots \ldots \ldots . \ldots \ldots$

3.6 Cylinder Off-Body Errors . . . . . . . . . . . . . . . . . . . . . . . 30

3.7 van de Vooren Airfoil Geometry . . . . . . . . . . . . . . . . . . 31

3.8 van de Vooren $C_{p}$ Distribution . . . . . . . . . . . . . . 31

4.1 Node Designations . . . . . . . . . . . . . . . . . . . . 37

4.2 DRM Matrices . . . . . . . . . . . . . . . . . . . . . . 40

4.3 Ellipse Geometry . . . . . . . . . . . . . . . . . . . . . . . 42

4.4 Iterative Procedure . . . . . . . . . . . . . . . . . . . 45

4.5 Iteration History $\ldots \ldots \ldots \ldots \ldots$

4.6 Cylinder O-Grid Near Body . . . . . . . . . . . . . . . . 55

4.7 Non-Conservative Cylinder Poisson Term . . . . . . . . . . . 56

4.8 Cylinder Domain Points . . . . . . . . . . . . . . . . . . 57

4.9 Suction Peak Error . . . . . . . . . . . . . . . . . . . . . . . . 58

4.10 Cylinder Iteration History … . . . . . . . . . . . . . 59

4.11 Cylinder Pressure Coefficient Distribution $\left(M_{\infty}=0.375\right) \ldots \ldots$

4.12 NACA 0012 Poisson Term . . . . . . . . . . . . . . . . . . . 60

4.13 Poison Tern Near Leading Edge . . . . . . . . . . . . . . . . . 61

4.14 Domain Points . . . . . . . . . . . . . . . . . . . . 61

4.15 NACA 0012 Iteration History . . . . . . . . . . . . . . . 62

4.16 NACA 0012 Cp Distribution $\left(\alpha=0, M_{\infty}=0.72\right) \ldots \ldots \ldots . . \ldots 2$

5.1 Point Cloud . . . . . . . . . . . . . . . . . . . . 65

5.2 Iterative Procedure . . . . . . . . . . . . . . . . . 68

5.3 Close-Up View of Point Distributions . . . . . . . . . . . . . . 70

5.4 Ghost Points Near a Leading Edge . . . . . . . . . . . . . . . . 79

5.5 Cylinder $C_{p}$ Distribution Using Ghost Node Method . . . . . . . . 80 
5.6 Visibility Method . . . . . . . . . . . . . . . . . 86

5.7 Bad Trailing Edge Results . . . . . . . . . . . . . . . . 86

5.8 Discontinous Potential Field . . . . . . . . . . . . . . . . . . . . 88

5.9 Variation of $\Phi$ Across Wake . . . . . . . . . . . . . . . . . . . . . . 89

5.10 Domain Cloud . . . . . . . . . . . . . . . . . . . . 92

5.11 Domain Point Discretization Error in Estimation of 1st and 2nd Derivatives . . . . . . . . . . . . . . . . . . . . . 93

5.12 Boundary Cloud . . . . . . . . . . . . . . . . . . . . . 94

5.13 Boundary Point Discretization Error _ . . . . . . . . . . . 96

6.1 Baseline Circular Cylinder Node Distribution . . . . . . . . . . . . 98

6.2 Close-Up View of Baseline Circular Cylinder Node Distribution . . . 99

6.3 Convergence History for Baseline Node Distribution . . . . . . . . . 100

6.4 Upper Surface Pressure Coefficient for the Baseline Node Distribution 100

6.5 Normal Velocity Component on Cylinder Surface . . . . . . . . . . 101

6.6 Evolution of Suction Peak Velocity Error . . . . . . . . . . . . . . . 102

6.7 Comparison of Local Cloud Configurations . . . . . . . . . . . . 103

6.8 Outer Boundary Setup . . . . . . . . . . . . . . . . . . . . 105

6.9 Suction Peak Error as a Function of the Farfield Radius . . . . . . . . 106

6.10 Comparison of Cylinder Node Distributions . . . . . . . . . . 108

6.11 Comparison with Van Dyke for Varying $M_{\infty} \ldots \ldots \ldots \ldots \ldots$

6.12 Supercritical Pressure Coefficient Distribution . . . . . . . . . . . 110

6.13 Baseline Airfoil Node Distribution . . . . . . . . . . . . . . . . 112

6.14 Close-Up View of Baseline Airfoil Node Distribution . . . . . . . . . . 112

6.15 Non-Lifting Case Iteration History _. . . . . . . . . . . . . . . 114

6.16 Pressure Coefficient Distribution for Non-Lifting Case $\left(M_{\infty}=0.72\right)$. 115

6.17 Normal Velocity Component for Non-Lifting Case . . . . . . . . . 115

6.18 Oscillatory Pressure Coefficient Distribution . . . . . . . . . . 116

6.19 Maximum Velocity Evolution for Non-Lifting Case . . . . . . . . . . . 117

6.20 Comparison of $C_{p}$ on Upper and Lower Surface for Baseline Distribution118

6.21 Comparison of Local Cloud Configurations at Leading Edge . . . . . 119

6.22 Pressure Coefficient Near Suction Peak as a Function of the Number of Cloud Support Points . . . . . . . . . . . . . . . . . . . . 119

6.23 Comparison of $C_{p}$ on Upper Surface for Different Farfield Radii . . . 121

6.24 Comparison of Upper Surface $C_{p} \ldots \ldots \ldots \ldots \ldots$

6.25 Comparison of Node Distributions on Airfoil . . . . . . . . . . 123

6.26 Comparison of Upper Surface $C_{p}$ as Function of Growth Ratio . . . . 124

6.27 Lifting Case Iteration History . . . . . . . . . . . . . . . . 125

6.28 Evolution of Flow Characteristics for Lifting Airfoil Case . . . . . . 126

6.29 Pressure Coefficient Distribution for Lifting Case . . . . . . . . . 127

6.30 Normal Velocity Component for Lifting Case . . . . . . . . . . . 127 


\title{
Nomenclature
}

\author{
Alphabetic \\ [A] System matrix \\ GFD matrix \\ $[B] \quad$ System matrix \\ GFD matrix \\ $b \quad$ Poisson term \\ $\{b\} \quad$ GFD vector \\ $C F D \quad$ Computational Fluid Dynamics \\ $C_{p} \quad$ Pressure coefficient \\ $[C] \quad$ GFD matrix \\ c Point location factor \\ Radial basis function free parameter \\ $c_{l} \quad$ Sectional lift coefficient \\ $D R M \quad$ Dual Reciprocity Method \\ $[D] \quad$ GFD matrix \\ $d_{m} \quad$ Mean distance \\ $\{d\} \quad$ GFD vector \\ $\hat{e}_{r} \quad$ Unit vector in radial direction \\ $[F] \quad$ Radial basis function matrix \\ $f \quad$ Radial basis function associated with the Laplacian \\ GFD Generalized Finite Difference \\ $[G] \quad$ Influence coefficient matrix \\ $[H] \quad$ Influence coefficient matrix \\ $h \quad$ Characteristic length \\ $k \quad$ Scaling factor \\ $L \quad$ Number of domain nodes \\ $l \quad$ Highest derivative order \\ M Mach number \\ $m \quad$ Binomial coefficient term \\ $\max \quad$ maximum \\ $N \quad$ Number of collocation points
}




\begin{tabular}{|c|c|}
\hline$N_{b}$ & Number of boundary nodes \\
\hline$N_{t}$ & Total number of nodes \\
\hline$\{n\}$ & GFD vector \\
\hline$n b$ & Number of boundary points \\
\hline$n s$ & Total number of cloud support points \\
\hline $\mathbf{n}$ & Normal vector \\
\hline$\left(n_{x}, n_{y}\right)$ & Cartesian components of the normal vector \\
\hline$P$ & Point \\
\hline$P D E$ & Partial Differential Equation \\
\hline$p$ & $\begin{array}{l}\text { Inverse distance weighting function factor } \\
\text { Order of a derivative }\end{array}$ \\
\hline Q & Total velocity vector \\
\hline $\mathrm{Q}_{\infty}$ & Freestream velocity vector \\
\hline$[\hat{Q}]$ & Approximate particular solution normal derivative matrix \\
\hline$q$ & Binomial coefficient term \\
\hline$q_{n}$ & Janzen-Rayleigh series coefficient \\
\hline$q^{*}$ & Normal derivative of Green's function \\
\hline$\hat{q}$ & Approximate normal derivative of the particular solution \\
\hline$R$ & Cylinder radius \\
\hline & Residual \\
\hline$\{R\}$ & Vector of Dual Reciprocity Method terms \\
\hline$R B F$ & Radial Basis Function \\
\hline$R H S$ & Right Hand Side \\
\hline$r$ & Euclidian distance \\
\hline & Radial direction \\
\hline & Ratio of mean distances \\
\hline$S$ & Surface \\
\hline$s$ & Integration dummy variable \\
\hline$t$ & Time \\
\hline & Total number of Taylor series terms \\
\hline$\left(U_{r}, U_{\theta}\right)$ & Polar velocity components \\
\hline$(U, V)$ & Total velocity components \\
\hline$\left(U_{\infty}, V_{\infty}\right)$ & Freestream velocity components \\
\hline$[\hat{U}]$ & Approximate particular solution matrix \\
\hline$u^{*}$ & Green's function \\
\hline$\hat{u}$ & Approximate particular solution \\
\hline$V$ & Volume \\
\hline$w$ & Derivative coefficient \\
\hline & Inverse distance weighting function \\
\hline$(x, y)$ & Cartesian coordinates \\
\hline$\{x\}$ & System vector \\
\hline
\end{tabular}




\begin{tabular}{ll}
$\{y\}$ & System vector \\
& \\
Greek & \\
$\alpha$ & Angle of attack \\
& Radial basis function interpolation coefficient \\
$\beta$ & Radial basis function interpolation coefficient \\
$\Gamma$ & Circulation \\
$\gamma$ & Ratio of specific heats \\
$\delta$ & Radius of infinitesimally small circle \\
$\epsilon$ & Airfoil thickness parameter \\
$\theta$ & Error \\
& angle measure in radians \\
$\mu$ & Radial component \\
$\Phi$ & Doublet Strength \\
$\Phi$ & Total velocity potential \\
$\phi$ & Freestream velocity potential \\
$\tilde{\phi}$ & Perturbation velocity potential \\
$\hat{\phi}$ & Homogeneous perturbation potential \\
$\rho$ & Non-homogeneous perturbation potential \\
$\sigma$ & Density \\
$\Psi$ & Source strength \\
$\omega$ & Trailing edge angle \\
& Arbitrary function \\
& Vorticity \\
& \\
\hline &
\end{tabular}

\section{Subscript}

A Lower wake point

a Airfoil

$B \quad$ Upper wake point

d Domain

g Ghost

$i \quad$ Index value

$j \quad$ Index value

$k \quad$ Index value

$l \quad$ Lower

$m \quad$ Maximum

ob Outer Boundary

u Upper 
$U$ velocity component

$v \quad V$ velocity component

$w \quad$ Wake

$0 \quad$ Cloud center

$\infty \quad$ Freestream

\section{Superscript}

$B \quad$ Boundary value

c coarse

$D \quad$ Domain value

d Cartesian direction

$f \quad$ fine

$m \quad$ Order of derivative

$n \quad$ Iteration

$1 x \quad$ First $\mathrm{x}$-derivative

$1 y \quad$ First y-derivative

$2 x \quad$ Second $\mathrm{x}$-derivative

$2 y \quad$ Second y-derivative

$+\quad$ Approached from upper side

- $\quad$ Approached from lower side

* $\quad$ Adjusted value 


\section{Chapter 1}

\section{Introduction}

\subsection{Motivation}

As the processing power and memory capacity of computers increases, the size and complexity of the problems that CFD techniques can solve has expanded. Solving for the turbulent unsteady flowfield about a complex three-dimensional configuration would have seemed to be a far off dream thirty years ago, but with improvements in computing capabilities such as parallel processing this dream has quickly become a reality. While it is true that the increase in computing power has so far experienced roughly exponential growth, the turnaround time for a CFD solution has not experienced such a drastic improvement. This disparity can be attributed to the fact that a significant amount of human input in the geometry definition and meshing phase is still required for an accurate and stable solution for problems of engineering interest.[1]

Jameson and Vassberg [2] state that in order for CFD software to play a primary role in the aerodynamic design process, the code needs to be able to satisfy three 
main requirements:

1: Sufficient and known level of accuracy

2: Acceptable computational and manpower costs

3: Fast turn around time

The first item is concerned with the ability for a code to predict the pertinent aerodynamic performance metrics with an acceptable amount of error. What is deemed an acceptable amount of error depends on what phase in the design process the CFD tool is being utilized. If the intent is to use the results from a CFD simulation without significant validation against wind tunnel or flight test data, then a high level of confidence in the results would be required. On the other hand if the CFD simulation is used as a design tool to explore the higher level trends in a parametric study instead of exact point performance metrics, then a lower fidelity analysis may be deemed acceptable.

The last two items are coupled since the turn around time influences the computational and manpower costs associated with the CFD simulation. The computational costs can be subdivided into the processing and memory requirements for a numerical simulation. A design study that explores the variation of multiple design variables requires a fast and efficient solution algorithm that can solve the large amount of cases required by such a study. The use of parallel processing often mitigates these issues by distributing the workload and memory requirements to a cluster of com- 
puters. The manpower costs associated with a CFD analysis is attributed to the expertise required from the user and the amount of time that is dedicated to setting up, executing, and analyzing the results from the simulation.

Much improvement has been made in terms of the fidelity of the analyses and the processing speed and memory capacity but there is still much work to be done in terms of the setup process. Specifically the meshing process can become the most time consuming task when analyzing a complex configuration. More often than not the responsibilities of setting up a mesh lie with an expert user because usually a significant amount of prior experience is needed to deal with issues such as CAD modeling errors and adequate grid refinement. Intuition is also important in terms of where more refinement is needed to accurately capture the important flow physics especially when a new mesh needs to be created to analyze changes in the geometry and freestream conditions. Therefore if a significant improvement in setup time can be achieved, there can be an equally significant speedup in the entire design cycle.

The panel method is an example of a numerical technique that provides a quick and easy to setup estimate for the aerodynamic characteristics of a configuration but with the sacrifice of a lower fidelity analysis. A panel method is restricted to solving for the inviscid incompressible flowfield, but the time to setup and run a panel code solution is significantly less than a standard CFD technique because only the surface geometry needs to be discretized. Most techniques that can solve for compressible flows such as the finite volume and finite difference method require the generation of 
a domain mesh. Since the meshing phase of a numerical simulation is often the most time consuming process, a numerical technique that can quickly and accurately solve for the compressible flowfield without the need for a mesh would be very desirable because it can provide the designer with a higher fidelity model without the added cost of a more extensive setup process.

\subsection{Scope of the Present Thesis}

The research conducted in this thesis began as an effort to extend the fidelity of a panel code without introducing the need for a domain mesh. This goal can be met by using a numerical technique called the Dual Reciprocity Method (DRM). This method allows for the compressiblity effects which would usually need to be captured by a domain grid to be estimated using an equivalent boundary integral. By transferring the compressiblity effects from the domain to the boundary, the boundary-only discretization property of a panel code could be retained. Unfortunately the Dual Reciprocity Method was deemed an impractical design tool due to the need for a priori knowledge of the correct solution to have the method converge to an accurate answer. Due to these shortcomings, a second method was developed called the Generalized Finite Difference Method. As the name implies it works in a similar manner as a standard finite difference scheme but without the need for connectivity data. This method proved to be adequate for solving for the compressible potential flow about two dimensional non-lifting and lifting geometries. 
In order to fully understand the concepts applied for both of these methods it is important to first address the principles behind compressible potential flows. The next chapter will show how these flows can be described by an area of mathematics known as potential theory. Since the Dual Reciprocity Method is essentially an augmented panel code, the basics behind a standard panel code capable of solving for the incompressible flowfield will be described in Chapter 3. Once these underlying principles have been established, Chapter 4 will address how to formulate the Dual Reciprocity Method for compressible potential flows and then examine two different test cases. The argument will be made for why the Dual Reciprocity Method did not prove to be a robust solution method which will then lead into the discussion of the Generalized Finite Difference method in Chapters 5 and 6 . Chapter 5 will discuss in detail the implementation of the Generalized Finite Difference method and compare its discretization error properties to a standard finite difference scheme. Once the underlying principles and implementation issues of the Generalized Finite Difference Method have been discussed in Chapter 5, Chapter 6 will provide some test case results. These will involve both non-lifting and lifting flows and will show node distribution sensitivity studies that are analogous to grid sensitivity studies that can be performed in standard CFD analyses. Chapter 7 will then draw conclusions about the two techniques by specifically highlighting unique research contributions, comparing the strengths and weaknesses of the two methods, and propose future work. 


\section{Chapter 2}

\section{Potential Aerodynamics}

Before any of the numerical techniques employed in this thesis can be discussed, the continuity equation and the role it plays in potential aerodynamics will be addressed. This chapter will discuss what assumptions are necessary for the existence of a velocity potential and how it can fully describe either compressible or incompressible inviscid flows in order to provide a mathematical foundation for which the subsequent chapters will be based on.

\subsection{The Full Potential Equation}

The continuity equation in differential form is a PDE that governs the conservation of mass for a fluid. In the Eulerian frame of reference it states that the net mass flow out of a fixed finite control volume is equal to the time rate of decrease of mass within the volume.[3] This principle can be expressed in differential form by the following equation:

$$
\frac{\partial \rho}{\partial t}+\nabla \cdot \rho \mathbf{Q}=0
$$


Equation (2.1) is known as the continuity equation in conservative form. It can also be expressed in non-conservative form by applying the chain rule to the second term.

$$
\frac{\partial \rho}{\partial t}+\mathbf{Q} \cdot \nabla \rho+\rho \nabla \cdot \mathbf{Q}=0
$$

Both equations make the same statement about the conservation of mass and can accurately be solved for problems with continuous changes in the flow properties. The difference lies in whether the problem would encounter discontinuities such as shocks. In this case Tannehill et. al.[4] purport that the use of the non-conservative form of the continuity equation can lead to numerical difficulties. In subsequent chapters it will be noted which form of the continuity equation is being solved.

The only assumption that has been placed on the flow is that it is a continuum. The continuity equation can be expressed in terms of a scalar function known as the velocity potential if the flow is also assumed to be irrotational which implies that the vorticity of the flow is zero.

$$
\omega=\nabla \times \mathbf{Q}=0
$$

The irrrotational assumption also implies that the flow field is inviscid since viscous effects introduce vorticity into the flowfield. Accoding to Crocco's theorem[5] a steady adiabatic flow with no energy exchange that has a non-zero vorticity also has an entropy gradient; therefore, the irrotational assumption also implies that the flowfield must also be isentropic. After these assumptions are made, a velocity potential can be established. The velocity field is simply the gradient of the velocity potential. The 
velocity potential can describe irrotational flows ranging from subsonic to supersonic Mach numbers as long as shocks are not present.

$$
\nabla \Phi=\mathbf{Q}
$$

The velocity field can be completely described by the scalar function $\Phi$ since it can be shown, by applying isentropic flow relations [6], that the density is also a nonlinear function of the velocity potential.

$$
\frac{\rho}{\rho_{\infty}}=\left[1+\frac{\gamma-1}{2} M_{\infty}^{2}\left(1-|\nabla \Phi|^{2}\right)\right]^{\frac{1}{\gamma-1}}
$$

Now that there is only one variable that needs to be solved, the continuity equation is the only governing equation needed to fully describe the flow. This equation is specifically known as the full potential equation and it describes the inviscid flowfield for problems with a freestream Mach number ranging from subsonic to supersonic.

$$
\frac{\partial \rho}{\partial t}+\nabla \cdot(\rho \nabla \Phi)=0
$$

This thesis will specifically deal with steady inviscid compressible flows. Therefore the time rate of change of the density will be zero. As with the continuity equation, the full potential equation can be expressed in non-conservative vector form by applying the chain rule to the second term in the steady form of Equation (2.6).

$$
\rho \nabla^{2} \Phi+\nabla \rho \cdot \nabla \Phi=0
$$

This equation can also be expressed in differential form:

$$
\rho\left(\frac{\partial^{2} \Phi}{\partial x^{2}}+\frac{\partial^{2} \Phi}{\partial y^{2}}\right)+\frac{\partial \rho}{\partial x} \frac{\partial \Phi}{\partial x}+\frac{\partial \rho}{\partial y} \frac{\partial \Phi}{\partial y}=0
$$


The full potential equation can also be expressed in a Poisson-type form which will be useful when solving compressible problems using the Dual Reciprocity Method. As with Equations (2.6) and (2.8), the Poisson-type equation can have either a conservative or non-conservative form.[7]

$$
\begin{aligned}
& \nabla^{2} \Phi=\nabla \cdot((1-\rho) \nabla \Phi) \text { conservative } \\
& \nabla^{2} \Phi=-\frac{\nabla \rho}{\rho} \cdot \nabla \Phi \text { non }- \text { conservative }
\end{aligned}
$$

Now that the governing PDE for full potential flows has been established it is necessary to enforce the correct boundary conditions in order to have a well posed problem. The velocity potential $(\Phi)$ is commonly broken up into a freestream $\left(\Phi_{\infty}\right)$ and a perturbation $(\phi)$ component. The freestream potential describes the flow at a boundary infinitely far away from the perturbing surface. The freestream flow is assumed to be uniform and at a constant angle of attack

$$
\Phi=\Phi_{\infty}+\phi
$$

The problem domain in an external aerodynamics problem consists of a farfield boundary $\left(S_{\infty}\right)$, a wall boundary $\left(S_{a}\right)$, and a wake cut plane $\left(S_{w}\right)$ as shown in Figure 2.1. The frame of reference used here will assume that the airfoil is stationary and aligned with the x-axis. The freestream flow approaches the airfoil at an angle attack $\alpha$ with respect to the $\mathrm{x}$-axis.

The airfoil surface can be considered to be a solid wall with the condition that the surface is also a streamline. As a consequence, this places a Neumman boundary 


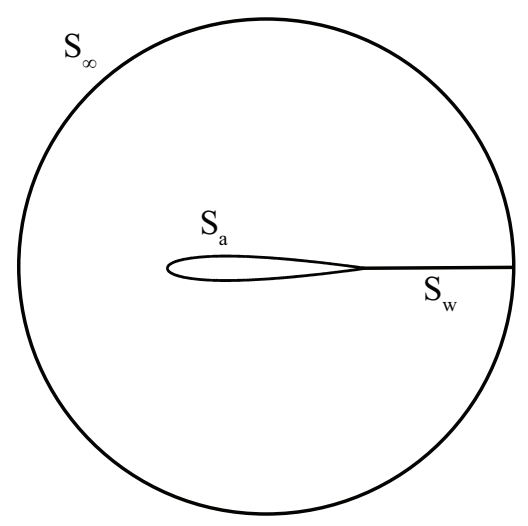

Figure 2.1: Problem Domain

condition on the velocity potential that states that no flow is normal to the surface.

$$
\begin{gathered}
\nabla \Phi \cdot \mathbf{n}=0 \\
\frac{\partial \Phi}{\partial \mathbf{n}}=0
\end{gathered}
$$

The circulation along with well posed boundary conditions need to be specified in order for a unique solution to be found for lifting flows; therefore, a wake surface is needed in order to correctly model the circulation. [3] The Kutta condition states that the value of the circulation is chosen so that the flow leaves the trailing edge of the airfoil smoothly. This means that for an airfoil with a finite trailing edge angle, such as the NACA 0012 studied in this thesis, the trailing edge is also a stagnation point. The wake can be modeled by a constant strength doublet distribution emanating from the trailing edge or equivalently a point vortex located anywhere within the airfoil. The strength of the doublet distribution or equivalently the strength of the point vortex is calculated by applying the Kutta condition at the trailing edge. This is commonly enforced by calculating the jump in potential between a point just above 
the trailing edge and a point just below the trailing edge of the airfoil.[8]

$$
\mu_{w}=\Gamma=\Phi_{u}-\Phi_{l}
$$

A Dirichlet condition is specified at the farfield boundary. The velocity potential at this boundary $\left(\Phi_{o b}\right)$ is composed of a superposition of a uniform velocity potential and the velocity potential induced by the point vortex that is enforcing the Kutta condition. [9]

$$
\Phi_{o b}=U_{\infty} x+V_{\infty} y+\frac{\Gamma}{2 \pi} \theta
$$

If the farfield boundary is sufficiently far away from the airfoil, the velocity is the freestream value because the perturbation velocities decay as $r \rightarrow \infty$ where $r$ is the Euclidean distance from a point on the surface of the airfoil to a point on the farfield boundary.

\subsection{Laplace's Equation}

Panel methods are used to solve for the incompressible flowfield which is governed by a simplified version of the Poisson-type form of the full potential equation. If the flow is assumed to be incompressible, then the density is constant everywhere in the flowfield. Therefore the time rate of change of the density is zero and the density term can be taken out of the gradient operator in Equation (2.6).

$$
\rho \nabla \cdot \nabla \Phi=0
$$


This equation can be expressed as the Laplacian of the velocity potential equal to zero.

$$
\nabla^{2} \Phi=0
$$

The same boundary conditions from the previous section still apply but now the flowfield is governed by a linear PDE which is called Laplace's equation. The term Laplace's equation governs a multitude of different physical and mathematical models and the study of solutions to this equation is known as Potential Theory.[10] Due to the linearity of the problem, the principle of superposition applies. Therefore the solution can be split up into a freestream and perturbation component. It will be explained in the next chapter how Laplace's equation can be solved by panel codes. The next chapter will specifically discuss in detail how to formulate and implement a panel code. Then these methods will be extended in Chapter 4 to solve the nonlinear full potential equation using a meshless technique called a the Dual Reciprocity Method. 


\section{Chapter 3}

\section{Panel Methods}

Panel Methods are used to solve problems of engineering interest that are adequately described by potential theory. Potential theory is a mathematical concept that can describe a multitude of physical models. This thesis will focus on potential theory and panel methods as applied to external aerodynamics.

Aerodynamic problems can be modeled by potential theory when the flowfield is assumed to be irrotational. If the flow is also assumed to be steady and incompressible, the continuity equation is reduced to Laplace's equation for the velocity potential. A popular numerical method for solving aerodynamic problems governed by Laplace's equation is the boundary integral method developed by Hess and Smith.[11] It is commonly known today as the panel method, yet Smith cited that while developing the code at Douglas aircraft, they called it the Neumann method due to the boundary conditions being imposed.[12] What makes the panel method a popular aerodynamic design tool is its ability to describe the flow on the surface and domain of a threedimensional complex geometry by only requiring the discretization of the surface. 
The panel method, also known as the boundary element method in other engineering disciplines, derives its name from the fact that the original formulation involved integrating an arbitrary distribution of the velocity potential along flat panels that discretized the geometry surface. This method provides a quick and inexpensive estimate of the velocity field when compared to CFD solutions due to the ease of discretization. It is a powerful tool when analyzing problems that can be accurately approximated by the inviscid and incompressible assumption, such as low-speed flows at small angles of attack.

This chapter will start by outlining the theory behind panel methods and then lead to how this theory is applied to solving problems of engineering interest. The presentation of the theory will focus on 2D flows, but it is straightforward to extend to a third dimension. The main difference between 2D and 3D calculations are the numerical subtleties associated with correctly modeling lifting flows. The first section will demonstrate how mathematical identities are used to transform Laplace's equation from a PDE into a boundary integral equation. Once the theory is established the numerical implementation of the problem will be discussed in detail. In the second section some numerical examples will be examined to verify that the panel code written for this thesis yields accurate results. 


\subsection{The Boundary Integral Equation}

As stated previously, panel codes are a popular design tool due to the requirement to only discretize the problem boundary instead of the entire domain. The boundaryonly nature of this method is made possible by expressing the solution as a boundary integral equation. This section will outline the mathematical principles that makes the boundary only nature of a panel method possible.

\subsubsection{Mathematical Formulation}

Since Laplace's equation describes a variety of different problems, different scientific and engineering communities have established differing methods and nomenclature for deriving essentially the same boundary integral equation. This equation can be derived either as a weighted residual statement or an application of one of Green's identities known as the reciprocity theorem. $[13,10]$ There is also a slight nuance between what kind of quantities are being integrated on the boundary itself. In the aerodynamics community distributions of singularities such as sources, doublets, and vortices are integrated in order to solve the problem whereas other disciplines integrate distributions of the potential itself. The use of singularity distributions is commonly known as the indirect boundary element method whereas the solution of the potential function is called the direct boundary element method.[13]

The aerodynamics community commonly uses the indirect method over the direct

method because these singularity distributions hold a physical meaning whereas a 
source or a doublet may not have a physical significance for other types of problems. For this thesis the boundary integral equation will be presented in the direct boundary element form by using the reciprocity theorem because it is more convenient to express it in this way in subsequent chapters. A review of the derivation using an indirect method can be found in Reference [8].

The reciprocity theorem is also known as Green's Third Identity which is a special case of the Divergence Theorem or as the vector form of integration by parts. It states that the Laplacian of a scalar function multiplied by its associated Green's function integrated throughout a domain is equivalent to a boundary integral of the potential and the Green's function.

$$
\begin{gathered}
\int_{V} u^{*} \nabla^{2} \Phi d V=\int_{S}\left(q^{*} \Phi-u^{*} \frac{\partial \Phi}{\partial \mathbf{n}}\right) d S=0 \\
u^{*}=\frac{1}{2 \pi} \ln \left(\frac{1}{r}\right) \\
q^{*}=\frac{\partial u^{*}}{\partial \mathbf{n}}
\end{gathered}
$$

The Green's function is also known as the fundamental solution to Laplace's equation. $r$ is the Euclidian distance between a point on the surface and the point of interest and $n$ is the unit normal vector pointing away from the domain as shown in Figure 3.1.

For the purposes of this thesis, the subsequent derivations will involve calculating the perturbation potential instead of the entire potential. As stated earlier, the principle of superposition makes this possible. Therefore the corresponding boundary 


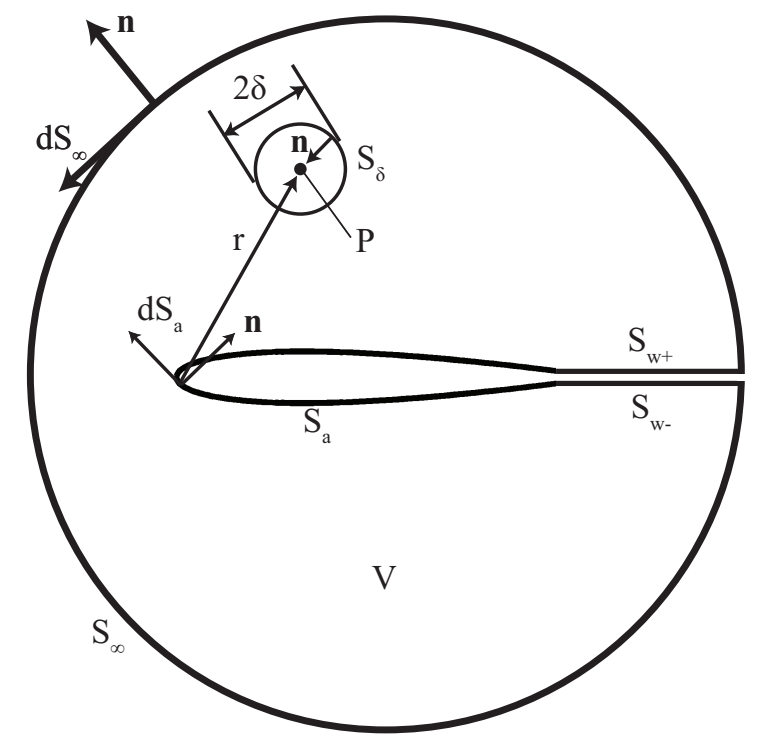

Figure 3.1: Integration Paths

integral will only involve perturbation velocity values.

$$
\int_{S}\left(q^{*} \phi-u^{*} \frac{\partial \phi}{\partial \mathbf{n}}\right) d S=0
$$

The problem domain, as shown in Figure 3.1, which is reproduced from Katz and Plotkin [8], consists of four boundaries: the airfoil surface, the wake cut plane, the farfield boundary, and finally an infinitesimally small circle of radius $\epsilon$ surrounding the point $P$ where it is desired to know the velocity potential. The normal vector points out of the domain and the integration path is determined by traversing in a direction where the normal vector is always pointing to the right. This corresponds to a clockwise path for the airfoil surface and an counter-clockwise path for the farfield. The boundary integral is then split up into the four portions and each is evaluated separately.

$$
\int_{S}\left(q^{*} \phi-u^{*} \frac{\partial \phi}{\partial \mathbf{n}}\right) d S=\int_{S_{\delta}+S_{w}+S_{\infty}+S_{a}}\left(q^{*} \phi-u^{*} \frac{\partial \phi}{\partial \mathbf{n}}\right) d S
$$


The surface integral surrounding the point $P$ is evaluated using a polar coordinate system. This coordinate system allows for a simple evaluation of the surface integral since $\mathbf{n}=-e_{r}, \frac{\partial \phi}{\partial \mathbf{n}}=-\frac{\partial \phi}{\partial r}$, and $q^{*}=-\frac{1}{2 \pi} \frac{\partial}{\partial r}\left(\ln \frac{1}{r}\right)=\frac{1}{2 \pi r}$. This results in the integral

$$
\int_{S_{\delta}}\left(q^{*} \phi-u^{*} \frac{\partial \phi}{\partial \mathbf{n}}\right) d S=\int_{S_{\delta}}\left(\frac{\phi}{2 \pi r}+u^{*} \frac{\partial \phi}{\partial r}\right) d S
$$

As $\delta \rightarrow 0$, it is assumed that the potential and its derivatives are well behaved in the infinitesimally small region surrounded by the circle. This assumption causes the second term in the second integral in (3.6) to be zero. The remaining term simplifies to

$$
\int_{S_{\delta}}\left(\frac{\phi}{2 \pi r}\right) d S=\left\{\begin{aligned}
\phi & \text { if } \mathrm{P} \text { is in } \mathrm{V} \\
\frac{1}{2} \phi & \text { if } \mathrm{P} \text { is on a smooth portion of } \mathrm{S}
\end{aligned}\right.
$$

The wake cut plane is assumed to be thin so that $\frac{\partial \phi}{\partial n}$ is continuous across the wake. This implies that no fluid dynamic loads can be supported by the wake.[8] These assumptions simplify the integral along the wake to

$$
\int_{S_{w}}\left(q^{*} \phi-u^{*} \frac{\partial \phi}{\partial \mathbf{n}}\right) d S=\int_{S_{w}}\left(q^{*} \phi\right) d S
$$

Since the problem was chosen to be solved in terms of the perturbation potential, the integral along the farfield boundary will vanish as long as certain regularity conditions are met. Assuming that the farfield boundary is a circle centered at the middle of the airfoil, the regularity condition states that the integrand in Equation (3.9) must approach zero as the farfield boundary radius becomes infinitely long.[14]

$$
\lim _{r_{\infty} \rightarrow \infty} \int_{S_{\infty}}\left(q^{*} \phi-u^{*} \frac{\partial \phi}{\partial n}\right) d S=0
$$


The Green's function associated with the 2D Laplace's equation, $u^{*}$, behaves at most $O(\ln r)$ and its derivative, $q^{*}$, behaves at most $O\left(r^{-1}\right)$ as $r_{\infty} \rightarrow \infty$. As stated earlier, the principle of superposition implies that the flow field around a geometry such as an airfoil can be composed of a series of elementary solutions such as sources, doublets, and vortices. For any of these singularities $\phi$ behaves at most as $\ln r$ and $\frac{\partial \phi}{\partial \mathbf{n}}$ behaves as $r^{-1}$; therefore, it is confirmed that the regularity conditions are met when solving the problem in terms of the perturbation potential because the terms in the integrand would cancel each other out. This regularity condition greatly simplifies the analysis by implicitly satisfying the farfield boundary condition without the need to model it with an explicitly defined farfield boundary geometry.

Now all the separate integrals can be combined to form the complete boundary integral equation.

$$
\begin{gathered}
c \phi(P)+\int_{S_{a}}\left(q^{*} \phi-u^{*} \frac{\partial \phi}{\partial \mathbf{n}}\right) d S+\int_{S_{w}}\left(q^{*} \phi\right) d S=0 \\
c=\left\{\begin{array}{l}
1 \text { if } \mathrm{P} \text { is in } \mathrm{V} \\
\frac{1}{2} \text { if } \mathrm{P} \text { is on a smooth portion of } \mathrm{S}
\end{array}\right.
\end{gathered}
$$

Determining $\phi(P)$ now only involves solving the boundary integral equation along the airfoil and wake cut plane. As stated previously, the wake can be modeled as a semi-infinite constant strength doublet distribution with a strength that is equal to the jump in velocity potential between a point on the upper side of the trailing edge and a point on the lower side of the trailing edge. This implies that there is a coupled relationship between the potential along the wake cut plane and the potential on the 
airfoil surface. Since the doublet strength is assumed to be constant, it can be taken out of the integrand, which results in

$$
c \phi(P)+\int_{S_{a}}\left(q^{*} \phi-u^{*} \frac{\partial \phi}{\partial \mathbf{n}}\right) d S+\mu_{w} \int_{S_{w}} q^{*} d S=0
$$

Equation (3.11) shows that there are three unknowns associated with the boundary integral problem: the potential on the airfoil surface, the flux of the velocity potential normal to the airfoil surface, and the wake doublet strength. Since this problem is solved in terms of the perturbation potential, $\frac{\partial \phi}{\partial \mathbf{n}}$ can be easily determined by applying the solid wall boundary condition.

$$
\begin{gathered}
\frac{\partial \Phi}{\partial \mathbf{n}}=\frac{\partial \Phi_{\infty}}{\partial \mathbf{n}}+\frac{\partial \phi}{\partial \mathbf{n}}=0 \\
\frac{\partial \phi}{\partial \mathbf{n}}=-\frac{\partial \Phi_{\infty}}{\partial \mathbf{n}}
\end{gathered}
$$

If the freestream flow is approaches the airfoil at an angle of attack $\alpha$, the freestream potential and its flux normal to the airfoil surface are simply

$$
\begin{gathered}
\Phi_{\infty}=\mathbf{Q}_{\infty} \cdot \mathbf{x}=U_{\infty} x+V_{\infty} y \\
\frac{\partial \Phi_{\infty}}{\partial \mathbf{n}}=\mathbf{Q}_{\infty} \cdot \mathbf{n}=U_{\infty} n_{x}+V_{\infty} n_{y}
\end{gathered}
$$

where $n_{x}$ and $n_{y}$ are the $x$ and $y$ components of the normal vector on the airfoil surface.

Since the problem is posed with a Neumann boundary condition, the boundary integral equation in mathematical terms is a Neumann problem. The presentation of this derivation was done differently than what is usually seen in the panel code 
community, and it is important to note the differences in nomenclature. First of all, what is called a Neumann problem in this thesis is actually called a source and doublet panel method with Dirichlet boundary conditions in the normal vernacular used in the aerodynamics community. A detailed discussion of the source and doublet method can be found in Reference [8] pg 290. The reason behind this confusing contradiction of terms is the fact that most textbooks on the subject solve the problem in terms of the indirect method instead of the direct method. When deriving the same equation using the indirect method the $\phi$ terms in Equation (3.11) are essentially the doublet

strengths $(\mu)$ and the $\frac{\partial \phi}{\partial \mathbf{n}}$ terms are the source strengths $(\sigma)$. Solving the gradient of Equation (3.11) dotted with the normal vector irrespective of whether the potential or its flux is known on the surface would be called a Neumann problem in the panel code community.

\subsubsection{Numerical Implementation}

The numerical implementation of a panel method uses a discretized version of the surface and numerically calculates the boundary integrals along each subdivided portion of the surface. Most methods use flat panels to discretize the surface as shown in Figure 3.2. Defined on each panel is a distribution of the potential and its flux which can be of any order. Most panel methods use either constant or linear distributions of the potential and flux. This thesis implements a constant distribution for both the potential and it's flux on the boundary. Therefore there is only one set of unknowns for each panel. The boundary conditions are usually enforced at points on 
the center of each panel. These boundary condition enforcement points are commonly known as a collocation points in the aerodynamics community. The normal vector is defined as the local unit vector normal to each of the $N$ panels as shown in Figure

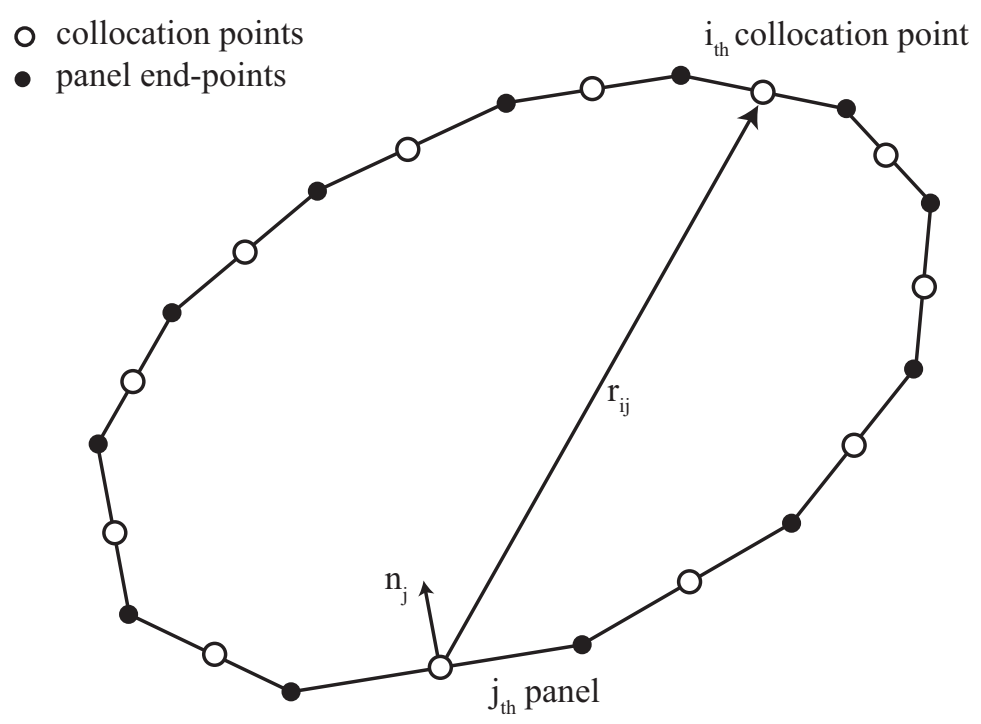

Figure 3.2: Surface Discretization

The discretized form of Equation (3.11) relates the potential at a point $i$ to the sum of all the integrals along each of the $j$ panels.

$$
\begin{gathered}
c_{i} \phi_{i}+\sum_{j=1}^{N} \int_{S_{j}} \phi_{j} q_{i j}{ }^{*} d S_{j}-\sum_{j=1}^{N} \int_{S_{j}} u_{i j}{ }^{*} \frac{\partial \phi_{j}}{\partial \mathbf{n}_{j}} d S_{j}+\mu_{w} \int_{S_{w}} q^{*} d S=0 \\
u_{i j}^{*}=\frac{1}{2 \pi} \ln \left(\frac{1}{r_{i j}}\right) \\
q_{i j}^{*}=\frac{\partial u_{i j}{ }^{*}}{\partial \mathbf{n}_{j}}
\end{gathered}
$$

Assuming that the distribution of the potential and it's flux is constant along each 
panel, these values can be taken out of the integrand.

$$
c_{i} \phi_{i}+\sum_{j=1}^{N} \phi_{j} \int_{S_{j}} q_{i j}^{*} d S_{j}-\sum_{j=1}^{N} \frac{\partial \phi_{j}}{\partial \mathbf{n}} \int_{S_{j}} u_{i j}^{*} d S_{j}+\mu_{w} \int_{S_{w}} q^{*} d S=0
$$

Most aerodynamic problems are only concerned with finding the velocity distribution on the surface. This means that the number of $i$ points are the same as the number of $j$ points. The integration factor $c_{i}=\frac{1}{2}$ because the potential is being calculated only on the surface. This also means that $\sum_{j=1}^{N} \int_{S_{j}} q_{i j}^{*} d S_{j}$ and $\sum_{j=1}^{N} \int_{S_{j}} u_{i j}^{*} d S_{j}$ can be represented by $[N \times N]$ matrices, where $\mathrm{N}$ is the number of panels. These matrices are commonly known as influence coefficient matrices because each row can be considered the influence of all the $j$ panels on the $i_{t h}$ collocation point. Each of the elemental integrations can be calculated using analytical integration as described in Appendix A or numerically by using Gaussian quadrature. $[8,15]$

$$
\begin{aligned}
& {\left[\bar{H}^{B}\right]=\left[\begin{array}{ccc}
\int_{S_{1}} q_{11}^{*} d S_{1} & \cdots & \int_{S_{N}} q_{1 N}^{*} d S_{N} \\
\vdots & \ddots & \vdots \\
\int_{S_{1}} q_{N 1}^{*} d S_{1} & \cdots & \int_{S_{N}} q_{N N}^{*} d S_{N}
\end{array}\right]} \\
& {\left[G^{B}\right]=\left[\begin{array}{ccc}
\int_{S_{1}} u_{11}^{*} d S_{1} & \cdots & \int_{S_{N}} u_{1 N}^{*} d S_{N} \\
\vdots & \ddots & \vdots \\
\int_{S_{1}} u_{N 1}^{*} d S_{1} & \cdots & \int_{S_{N}} u_{N N}^{*} d S_{N}
\end{array}\right]}
\end{aligned}
$$

The inclusion of the wake influence adds an extra column to $\left[\bar{H}^{B}\right]$ and $\left[G^{B}\right]$. These added columns are

$$
\begin{gathered}
\bar{H}_{i, N+1}^{B}=\int_{S_{w}} q_{i}^{*} d S \\
G_{i, N+1}^{B}=0
\end{gathered}
$$

Now there are $N$ equations and $N+1$ unknowns. To close the system of equations, 
an additional row is needed for the $\left[\bar{H}^{B}\right]$ and $\left[G^{B}\right]$ matrices. The added row in $\left[\bar{H}^{B}\right]$ is the numerical enforcement of the Kutta condition at the trailing edge.

Usually the boundary integration starts at the trailing edge panel on the lower surface and ends at the trailing edge panel on the upper surface. It is also assumed that these panels have collocation points sufficiently close to the trailing edge so that enforcing the Kutta condition at the collocation points introduces only minor errors in the wake strength calculation.

$$
\begin{gathered}
\phi_{N}-\phi_{1}-\mu_{w}=0 \\
\bar{H}_{N+1,1 \ldots N+1}^{B}=\left[\begin{array}{llllll}
1 & 0 & \cdots & 0 & 1 & -1
\end{array}\right]
\end{gathered}
$$

All the elements in the added row of the $\left[G^{B}\right]$ matrix are simply zero.

The boundary-only version of Equation (3.16) can now be fully represented as

$$
\frac{1}{2}\left\{\phi^{B}\right\}+\left[\bar{H}^{B}\right]\left\{\phi^{B}\right\}-\left[G^{B}\right]\left\{\frac{\partial \phi}{\partial \mathbf{n}}\right\}=0
$$

where $B$ emphasizes that the values only pertain to the boundary.

$$
\begin{gathered}
\left\{\phi^{B}\right\}=\left[\begin{array}{lll}
\phi_{1} \cdots & \phi_{N} & \mu_{w}
\end{array}\right]^{T} \\
\left\{\frac{\partial \phi}{\partial \mathbf{n}}\right\}=\left[\begin{array}{lll}
\frac{\partial \phi_{1}}{\partial \mathbf{n}} \cdots \frac{\partial \phi_{N}}{\partial \mathbf{n}} & 0
\end{array}\right]^{T}
\end{gathered}
$$

The first term in Equation (3.26) can be incorporated into the diagonal of the $\left[\bar{H}^{B}\right]$ matrix.

$$
\left[H^{B}\right]=\left\{\begin{array}{r}
\bar{H}_{i j}^{B} \text { if } \mathrm{i} \neq \mathrm{j} \\
\bar{H}_{i j}^{B}+\frac{1}{2} \text { if } \mathrm{i}=\mathrm{j}
\end{array}\right.
$$


The values of the potential on the surface can now be determined by solving the system of equations.

$$
\left\{\phi^{B}\right\}=\left[H^{B}\right]^{-1}\left[\left[G^{B}\right]\left\{\frac{\partial \phi}{\partial \mathbf{n}}\right\}\right]
$$

Now that the values of the potential are known on the surface of the airfoil, the surface velocities can be determined by taking derivatives of the potential in the tangential direction since the airfoil surface is a streamline. In the code written for this thesis these derivatives are approximated by the second order central difference scheme.

To determine the potential at $L$ domain points, Equation (3.16) is solved by building $[L \times N+1]$ sized $\left[\bar{H}^{D}\right]$ and $\left[G^{D}\right]$ matrices. These matrices have the same form as Equations (3.20) and (3.21) but are calculated for an $i=1 \ldots L$ amount of domain points instead of the $i=1 \ldots N$ boundary points.

$$
\left\{\phi^{D}\right\}=-\left[\mathbf{H}^{D}\right]\left\{\phi^{B}\right\}+\left[G^{D}\right]\left\{\frac{\partial \phi}{\partial \mathbf{n}}\right\}
$$

The off-surface velocities at the $L$ domain points can also be determined by taking the gradient of Equation (3.19).

$$
\nabla \phi_{i}+\sum_{j=1}^{N} \phi_{j} \int_{S_{j}} \nabla q_{i j}^{*} d S_{j}-\sum_{j=1}^{N} \frac{\partial \phi_{j}}{\partial \mathbf{n}} \int_{S_{j}} \nabla u_{i j}^{*} d S_{j}+\mu_{w} \int_{S_{w}} \nabla q^{*} d S=0
$$

The matrices associated with the fluxes are constructed in the same manner as (3.20) and (3.21).

$$
\left[\bar{H}_{u}^{D}\right]=\left[\begin{array}{cccc}
\int_{S_{1}} \frac{\partial q_{11}^{*}}{\partial x} d S_{1} & \cdots & \int_{S_{N}} \frac{\partial q_{1 N}^{*}}{\partial x} & \int_{S_{w}} \frac{\partial q_{1 w}^{*}}{\partial x} d S_{w} \\
\vdots & \ddots & \vdots & \vdots \\
\int_{S_{1}} \frac{\partial q_{L 1}^{*}}{\partial x} d S_{1} & \cdots & \int_{S_{N}} \frac{\partial q_{L N}^{*}}{\partial x} d S_{N} & \int_{S_{w}} \frac{\partial q_{L w}^{*}}{\partial x} d S_{w}
\end{array}\right]
$$




$$
\begin{aligned}
& {\left[\bar{H}_{v}^{D}\right]=\left[\begin{array}{cccc}
\int_{S_{1}} \frac{\partial q_{11}^{*}}{\partial y} d S_{1} & \cdots & \int_{S_{N}} \frac{\partial q_{1 N}^{*}}{\partial y} & \int_{S_{w}} \frac{\partial q_{1 w}^{*}}{\partial y} d S_{w} \\
\vdots & \ddots & \vdots & \vdots \\
\int_{S_{1}} \frac{\partial q_{L 1}^{*}}{\partial y} d S_{1} & \cdots & \int_{S_{N}} \frac{\partial q_{L N}^{*}}{\partial y} d S_{N} & \int_{S_{w}} \frac{\partial q_{L w}^{*}}{\partial y} d S_{w}
\end{array}\right]} \\
& {\left[G_{u}^{D}\right]=\left[\begin{array}{cccc}
\int_{S_{1}} \frac{\partial u_{11}^{*}}{\partial x} d S_{1} & \cdots & \int_{S_{N}} \frac{\partial u_{1 N}^{*}}{\partial x} & \int_{S_{w}} \frac{\partial u_{1 w}^{*}}{\partial x} d S_{w} \\
\vdots & \ddots & \vdots & \vdots \\
\int_{S_{1}} \frac{\partial u_{L 1}^{*}}{\partial x} d S_{1} & \cdots & \int_{S_{N}} \frac{\partial u_{L N}^{*}}{\partial x} d S_{N} & \int_{S_{w}} \frac{\partial u_{L w}^{*}}{\partial x} d S_{w}
\end{array}\right]} \\
& {\left[G_{v}^{D}\right]=\left[\begin{array}{cccc}
\int_{S_{1}} \frac{\partial u_{11}^{*}}{\partial y} d S_{1} & \cdots & \int_{S_{N}} \frac{\partial u_{1 N}^{*}}{\partial y} & \int_{S_{w}} \frac{\partial u_{1 w}^{*}}{\partial y} d S_{w} \\
\vdots & \ddots & \vdots & \vdots \\
\int_{S_{1}} \frac{\partial u_{L 1}^{*}}{\partial y} d S_{1} & \cdots & \int_{S_{N}} \frac{\partial u_{L N}^{*}}{\partial y} d S_{N} & \int_{S_{w}} \frac{\partial u_{L w}^{*}}{\partial y} d S_{w}
\end{array}\right]}
\end{aligned}
$$

The total velocity consists of the perturbation velocity calculated by the matrix equations and the freestream velocity.

$$
\begin{aligned}
& \left\{U^{D}\right\}=-\left[\bar{H}_{u}^{D}\right]\left\{\phi^{B}\right\}+\left[G_{u}^{D}\right]\left\{\frac{\partial \phi}{\partial \mathbf{n}}\right\}+\left\{U_{\infty}^{D}\right\} \\
& \left\{V^{D}\right\}=-\left[\bar{H}_{v}^{D}\right]\left\{\phi^{B}\right\}+\left[G_{v}^{D}\right]\left\{\frac{\partial \phi}{\partial \mathbf{n}}\right\}+\left\{V_{\infty}^{D}\right\}
\end{aligned}
$$

A method for solving the potential on the boundary and in the domain has now been presented. The solution procedure has been presented in a manner that implies that the boundary values need to be solved first, and then the potential at the domain points can be calculated by applying Equation (3.31). These seemingly separate systems of equations can actually be coupled together by using some matrix manipulation. Solving for the boundary and domain values simultaneously will prove to be helpful when describing the methods in the next chapter. Therefore the procedure for coupling the problem for non-lifting flows will now be described.

The $\left[H^{B}\right]$ and $\left[\bar{H}^{D}\right]$ as well as the $\left[G^{B}\right]$ and $\left[G^{D}\right]$ matrices can be combined into composite matrices. A new system of equations is defined so that the potential 
at the $N$ boundary and $L$ domain points can be simultaneously solved. A schematic of this system is shown below. The boundary and domain matrices are combined to

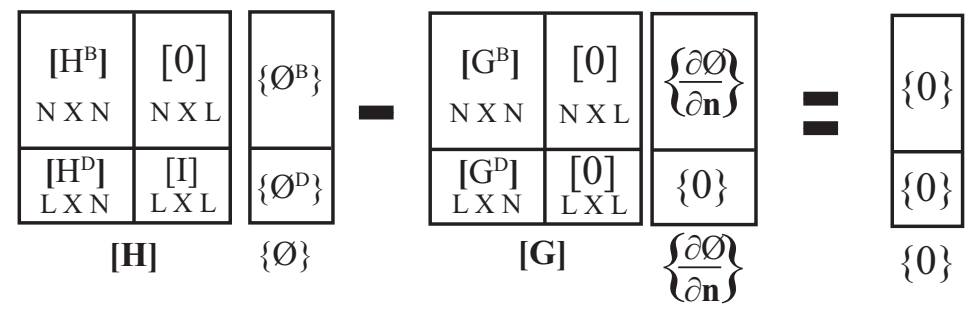

Figure 3.3: Coupled System Setup

form the total system $[H]$ and $[G]$ matrices with size $[N+L \times N+L]$. The $\left\{\phi^{B}\right\}$ and $\left\{\phi^{D}\right\}$ vectors are also combined to form an $\{N+L \times 1\}$ total system $\{\phi\}$ vector. Once these composite matrices and vectors are assembled, the system can be easily solved.

$$
\{\phi\}=[H]^{-1}\left[[G]\left\{\frac{\partial \phi}{\partial \mathbf{n}}\right\}\right]
$$

\subsection{Code Verification}

Some test cases were analyzed to confirm that both non-lifting and lifting flows can be accurately calculated by the panel method outlined in the previous sections. The non-lifting case compares the panel method results to the classic non-lifting circular cylinder problem. Lifting flows were verified by comparing the surface velocities of a van de Vooren airfoil at an angle of attack, which has an analytical solution by using conformal mapping.[8] 


\subsubsection{Circular Cylinder}

The circular cylinder problem has a very simple analytical solution for both the potential and velocities anywhere in the domain and on the surface. The flowfield is composed of a uniform flow and a point doublet located at the center of the cylinder. The analytical expression [3] for the potential and velocities is presented in its simplest form using a polar coordinate system as shown in Figure 3.4.

$$
\begin{gathered}
\Phi=U_{\infty} \cos \theta\left(r+\frac{R^{2}}{r}\right) \\
U_{r}=U_{\infty} \cos \theta\left(1-\frac{R^{2}}{r^{2}}\right) \\
U_{\theta}=-U_{\infty} \sin \theta\left(1+\frac{R^{2}}{r^{2}}\right) \\
C_{p}=1-4 \sin ^{2} \theta
\end{gathered}
$$

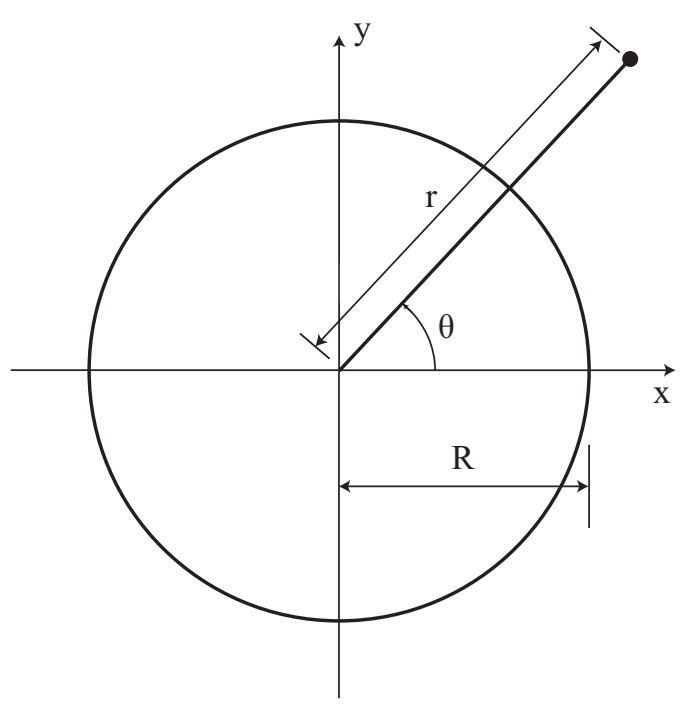

Figure 3.4: Circular Cylinder Geometry 
The surface of a 0.5 radius cylinder centered at $(0.5,0)$ was discretized by the panel method. The following plot shows the pressure coefficient distribution calculated with fifty panels uniformly discretizing the surface. The flow is symmetric about the y-axis so only the pressure coefficient distribution on the upper surface is shown. Agreement is excellent with the analytical solution.[3]

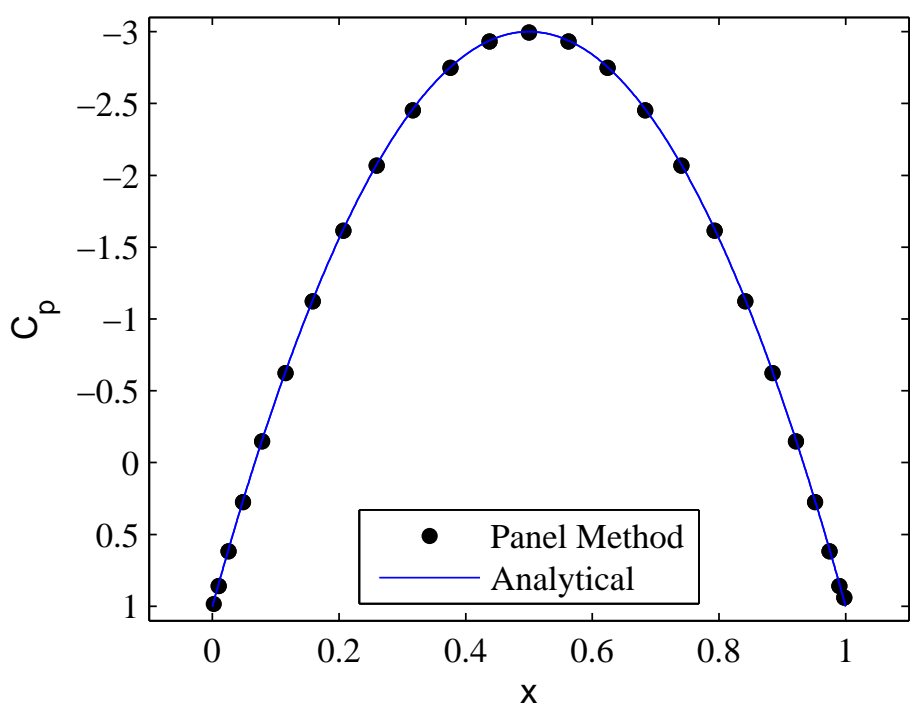

Figure 3.5: Circular Cylinder $C_{p}$ Distribution

An estimate of the off-body potential and velocity calculations was also investigated by computing the error at a point that is located at $(0.4,0.6)$, which is near the upper suction peak of the cylinder. This point was chosen because it is sufficiently close to the surface, which ensures that the perturbation potential and velocities are not negligible. As can be seen in Figure 3.6, the errors are relatively small for a number of panels $(\mathrm{N})$ greater than fifty. The errors were calculated as the absolute value of the difference between the panel solution and the analytical solution. 


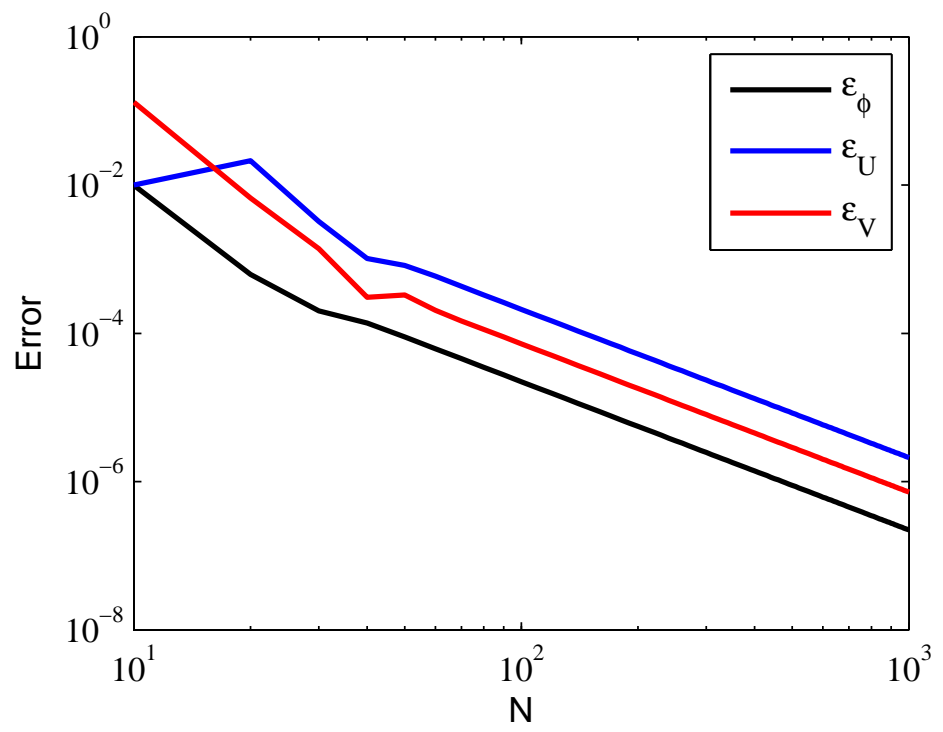

Figure 3.6: Cylinder Off-Body Errors

\subsubsection{Lifting Airfoil}

The van de Vooren airfoil is a valuable verification tool because the surface velocities can be analytically calculated for both non-lifting and lifting cases. [8] The analytical on-body velocities are calculated by first determining the velocities on an equivalent circular cylinder in the $h-g$ complex plane. These velocities are then mapped back into the cartesian $x-y$ plane by using a conformal mapping. The van de Vooren transformation applies to a class of airfoil shapes rather than a single profile. The trailing edge angle and thickness can be adjusted to define different airfoil profiles.

The verification case compares the pressure coefficient distribution on a van de Vooren airfoil at a two degree angle of attack with a thickness parameter $\epsilon=0.08325$, which corresponds to a $15 \%$ maximum thickness, and a trailing edge angle of $\tau=10^{\circ}$. 


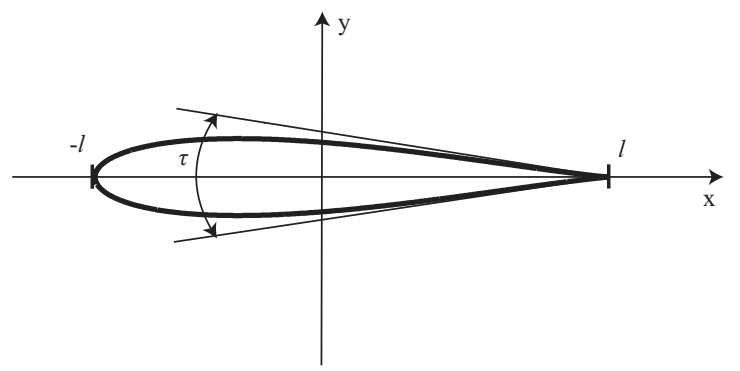

Figure 3.7: van de Vooren Airfoil Geometry

The geometry was discretized with 50 panels using a cosine spacing formula so that more panels are located near the leading and trailing edges. As can be seen in Figure 3.8 , there is very good agreement with the analytical solution.

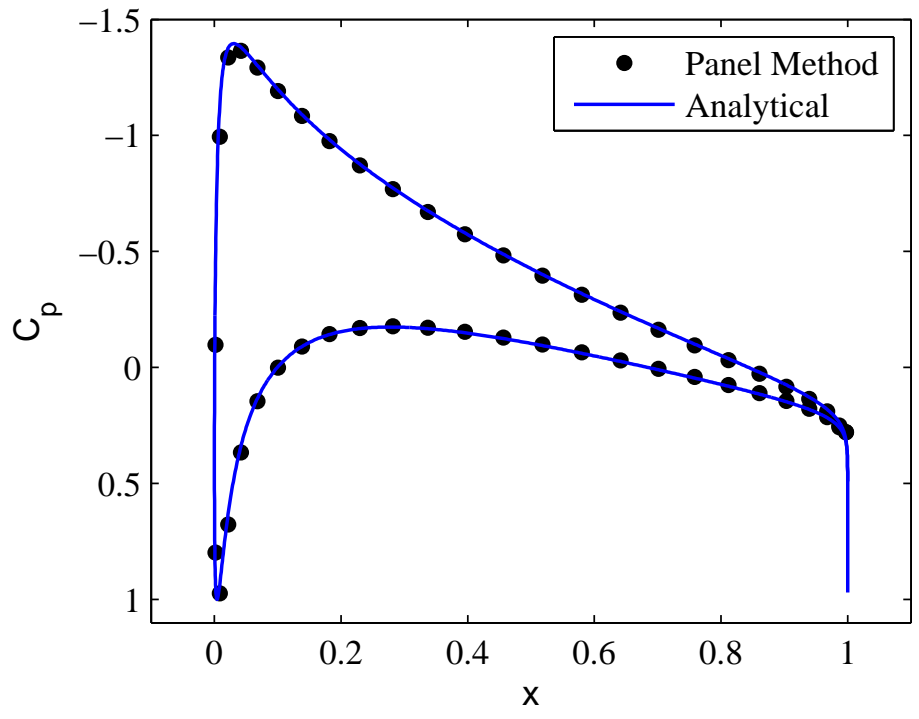

Figure 3.8: van de Vooren $C_{p}$ Distribution

To summarize, the panel method that was developed used constant distributions of the problem unknowns on flat panels to solve for the potential and velocity fields 
both on and off the surface of arbitrary shapes. The method was shown to accurately determine these flowfields for both lifting and non-lifting aerodynamic problems. The next chapter will discuss how the underlying principles of panel methods can be extended to solve for the compressible potential flowfield. 


\section{Chapter 4}

\section{The Dual Reciprocity Method}

The initial motivation for this thesis was to extend the utility of a panel code to model compressible flows. If the inviscid assumption remains, but the incompressible assumption no longer applies, the flow field can still be fully described by the scalar velocity potential function. The compressible flow assumption converts the linear Laplace equation into a non-linear Poisson-type equation.

$$
\nabla^{2} \phi=b(x, y, \Phi)
$$

The panel method as it was formulated in the previous chapter can no longer solve this problem. Researchers at the University of Stuttgart had developed a boundaryonly technique for solving flows governed by the full potential equation.[16, 7, 17] Theurer applied the method to two-dimensional transonic flows [16], then the method was extended to three-dimensional flows by Uhl et. al.[17] They accomplished this task by using the Dual Reciprocity Method (DRM), which was first formulated by Nardini and Brebbia.[18] The appealing characteristic of the DRM is that it allows for the numerical solution of an arbitrary Poisson-type equation using the same underlying 
principles of a panel method.

The initial plan for this thesis was to extend the methods developed by the research team at the University of Stuttgart and apply it to a three-dimensional unstructured panel code. The first step toward accomplishing this task was to reproduce the results from the University of Stuttgart research team. Once the underlying principles of the dual reciprocity method were well understood, research would be done for applying the method to a code written in an unstructured framework. This chapter will describe the efforts made to reproduce the work done by the German research team. Unfortunately, this research goal could not be fully realized and the reasons why will be examined.

The first section will layout the theoretical framework for how the dual reciprocity method transforms the domain integral of the nonlinear Poisson term into an equivalent boundary integral. Subsequent sections will explore how the dual reciprocity method can be applied to solving full potential flows.

\subsection{Formulation}

The dual reciprocity method (DRM) is a numerical method that can solve a variety of different Poisson-type problems. The definitive book on the Dual Reciprocity Method written by Partridge et. al. [19] first presents the DRM as applied to problems with a simple Poisson term such as constants and functions of geometry, then the book continues to apply the method to problems with increasing complexity. The 
same pedagogy used by Partridge et. al. will be applied in this thesis because it is the most straightforward way to introduce the DRM to a new reader on the subject. The next two subsections will specifically address two different types of Poisson-Type equations as described by Partridge and Brebbia.[20] These two types of problems are classified by the complexity of the Poisson term:

1: $b=b(x, y)$; where $\mathrm{b}$ is a constant or a known function of space

2: $\quad b=b(x, y, \phi) ;$ where $\mathrm{b}$ is function of space and the problem solution

\subsubsection{Simple Poisson Term}

Consider a Poisson equation with a right-hand side $b$ that is either a constant or a function of the problem geometry.

$$
\nabla^{2} \phi=b(x, y)
$$

The reciprocity theorem can still be applied to Equation (4.2) but unlike the panel method described in the previous chapter, the volume integral does not vanish. Solving the problem in this form sacrifices the boundary-only appeal of a panel code.

$$
c \phi(P)+\int_{S}\left(q^{*} \phi-u^{*} \frac{\partial \phi}{\partial n}\right) d S=\int_{V} b u^{*} d V
$$

Solving Equation (4.3) is the basis for Field Panel methods. Field Panel methods directly calculate the volume integral which means that a mesh needs to be constructed on both the boundary and domain of the problem.[21] 
It is assumed that the solution to the PDE can be split into a homogeneous and non-homogeneous portion.

$$
\begin{aligned}
& \nabla^{2} \hat{\phi}=0 \\
& \nabla^{2} \hat{\phi}=b
\end{aligned}
$$

The volume integral term in Equation (4.3) can now be expressed in terms of the particular solution.

$$
\int_{V} b u^{*} d V=\int_{V} \nabla^{2} \hat{\phi} u^{*} d V
$$

The reciprocity theorem can be applied a second time by converting the domain integral into an equivalent boundary integral. This transformation is powerful because it reduces the complexity of the problem by restoring the boundary-only nature of a panel method.

$$
c_{i} \phi_{i}+\int_{S}\left(q^{*} \phi-u^{*} \frac{\partial \phi}{\partial \mathbf{n}}\right) d S=c_{i} \hat{\phi}_{i}+\int_{S}\left(q^{*} \hat{\phi}-u^{*} \frac{\partial \hat{\phi}}{\partial \mathbf{n}}\right) d S
$$

Now there is one equation and four unknowns: $\phi, \frac{\partial \phi}{\partial \mathbf{n}}, \hat{\phi}$, and $\frac{\partial \hat{\phi}}{\partial \mathbf{n}}$. The total perturbation potential values $\phi$ or $\frac{\partial \phi}{\partial \mathbf{n}}$ can be eliminated as unknowns depending on whether a Dirichlet or Nuemman boundary condition is specified. Usually there is no easy way to determine an exact form for the particular solutions. Nardini and Brebbia [18] proposed the use of a linear combination of an analytical function known as a radial basis function $(\mathrm{RBF})$ to approximate the particular solutions. RBF's were chosen due to their ability to interpolate data on a scattered distribution of points. 
The RBF interpolant is used to approximate the Poisson term $b$ at a discrete set of $N$ points on the boundary and $L$ points in the domain. This scenario is depicted in the figure below for a problem with a bounded domain. Points are usually needed in the domain to improve the accuracy of the interpolation especially for problems that exhibit large gradients on the boundary or in the domain. The $N$ points on the boundary are chosen to be the collocation points that are generated during the paneling of the boundary geometry.

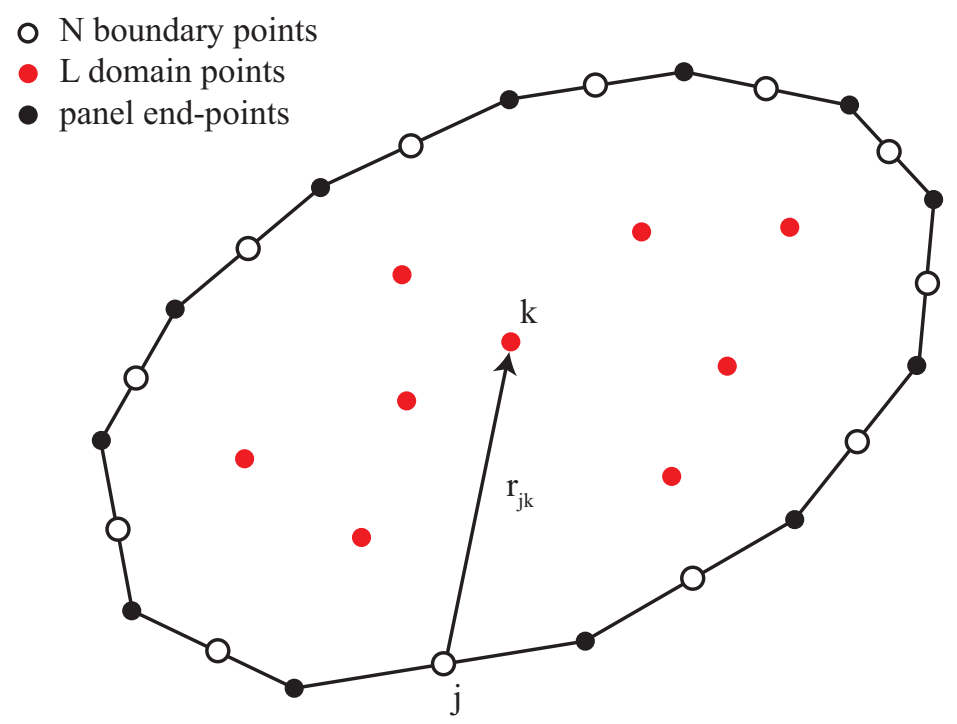

Figure 4.1: Node Designations

The radial basis function that was initially chosen by Nardini and Brebbia will be used in this section as an example, yet the literature offers a multitude of choices for the specific form of the RBF interpolant.[22] The Poisson term is directly approximated as a linear combination of the RBF and a set of initially unknown coefficients.

$$
\nabla^{2} \hat{\phi}_{j}=\sum_{k=1}^{N+L} f_{j k} \alpha_{k} \quad j=1 \ldots N
$$




$$
\begin{gathered}
f_{j k}=1+r_{j k} \\
r_{j k}=\sqrt{\left(x_{k}-x_{j}\right)^{2}+\left(y_{k}-y_{k}\right)^{2}}
\end{gathered}
$$

The particular solutions $\hat{\phi}$ and $\frac{\partial \hat{\phi}}{\partial n}$ can be approximated in a similar manner.

$$
\begin{gathered}
\hat{\phi}_{j}=\sum_{k=1}^{N+L} \hat{u}_{j k} \alpha_{k} \\
\frac{\partial \hat{\phi}_{j}}{\partial \mathbf{n}}=\sum_{k=1}^{N+L} \hat{q}_{j k} \alpha_{k}
\end{gathered}
$$

where $\hat{u}$ is related to $f$ by the Laplacian, which simplifies to a second order differential equation in polar coordinates for RBF's.

$$
\nabla^{2} \hat{u}=\frac{1}{r} \frac{d}{d r}\left(r \frac{d \hat{u}}{d r}\right)=f
$$

The equation can be solved by integrating $f$ form an interval of 0 to $r$.

$$
\hat{u}=\int_{0}^{r} \frac{1}{s}\left[\int_{0}^{t} t f(t) d t\right] d s
$$

For the case of $f=1+r$ the integral results in

$$
\hat{u}=\int_{0}^{r} \frac{1}{s}\left[\int_{0}^{t} t(1+t) d t\right] d s=\frac{r^{2}}{4}+\frac{r^{3}}{9}
$$

The normal derivative of the particular solution $\hat{q}$ can now be found by calculating the dot product of the gradient of $\hat{u}$ with the normal vector.

$$
\hat{q}=\frac{\partial \hat{u}}{\partial r} \frac{\partial r}{\partial x} \frac{\partial x}{\partial \mathbf{n}}+\frac{\partial \hat{u}}{\partial r} \frac{\partial r}{\partial y} \frac{\partial y}{\partial \mathbf{n}}=\frac{1}{r} \frac{\partial \hat{u}}{\partial r}\left(x n_{x}+y n_{y}\right)
$$

For the case of $\hat{u}=\frac{r^{2}}{4}+\frac{r^{3}}{9}$ this simplifies to

$$
\hat{q}=\left(\frac{1}{2}+\frac{r}{3}\right)\left(x n_{x}+y n_{y}\right)
$$


The unknown coefficients $\alpha_{k}$ can be easily determined since the Poisson term $b$ is a known function. First a square $[N+L \times N+L]$ matrix $[F]$ is constructed whose elements consist of the evaluations of $f$ at each of the $N+L$ points. Then a $\{N+L \times 1\}$ vector $\{b\}$ is constructed, which contains the evaluation of the Poisson term at each of the $N+L$ points. This results in the following system of equations

$$
[F]\{\alpha\}=\{b\}
$$

This equation is then solved for the vector $\alpha$ containing the $N+L$ coefficients used in Equations (4.8),(4.11), and (4.12).

$$
\{\alpha\}=[F]^{-1}\{b\}
$$

Now that the approximations for $\hat{\phi}$ and $\frac{\partial \hat{\phi}}{\partial \mathbf{n}}$ have been established, they can be substituted into Equation (4.7).

$$
c_{i} \phi_{i}+\int_{S}\left(q^{*} \phi-u^{*} \frac{\partial \phi}{\partial \mathbf{n}}\right) d S=\sum_{k=1}^{N+L} \alpha_{k}\left[c_{i} \hat{\phi}_{i k}+\int_{S}\left(q^{*} \hat{u}_{k}-u^{*} \hat{q}_{k}\right) d S\right]
$$

The integrals are calculated numerically using the same method described in the previous chapter.

$$
\begin{gathered}
c_{i} \phi_{i}+\sum_{j=1}^{N} \phi_{j} \int_{S_{j}} q^{*} d S_{j}-\sum_{j=1}^{N} \frac{\partial \phi_{j}}{\partial \mathbf{n}} \int_{S_{j}} u^{*} d S_{j}= \\
\sum_{k=1}^{N+L} \alpha_{k}\left[c_{i} \hat{u}_{i k}+\sum_{j=1}^{N} \hat{u}_{j k} \int_{S_{j}} q^{*} d S_{j}-\sum_{j=1}^{N} \hat{q}_{j k} \int_{S_{j}} u^{*} d S_{j}\right]
\end{gathered}
$$

When solving for the $N+L$ unknowns, the problem is expressed in matrix form as

$$
[H]\{\phi\}-[G]\left\{\frac{\partial \phi}{\partial \mathbf{n}}\right\}=[[H][\hat{U}]-[G][\hat{Q}]]\{\alpha\}
$$


The matrices $[H]$ and $[G]$ have the same meaning described in the previous chapter. The $i_{\text {th }}$ rows of the $\hat{\mathbf{U}}$ and $\hat{\mathbf{Q}}$ matrices represent the evaluations of their respective radial basis functions for approximating the particular solutions at the $i_{t h}$ point. Figure 4.2 below illustrates how all the matrices are assembled.

$$
\begin{aligned}
& \hat{U}_{i k}=\frac{r_{i k}{ }^{2}}{4}+\frac{r_{i k}{ }^{3}}{9} \\
& \hat{Q}_{i k}=\left\{\begin{array}{rr}
\left(\frac{1}{2}+\frac{r_{i k}}{3}\right)\left[\left(x_{k}-x_{i}\right) n_{x i}+\left(y_{k}-y_{i}\right) n_{y i}\right] & \mathrm{i} \leq \mathrm{N} \\
0 & \mathrm{i}>\mathrm{N}
\end{array}\right.
\end{aligned}
$$

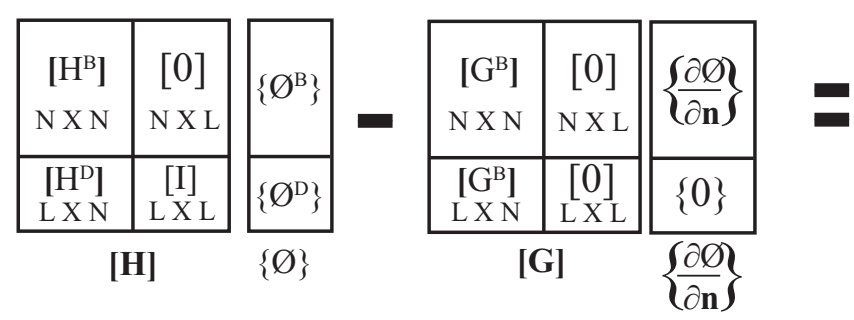

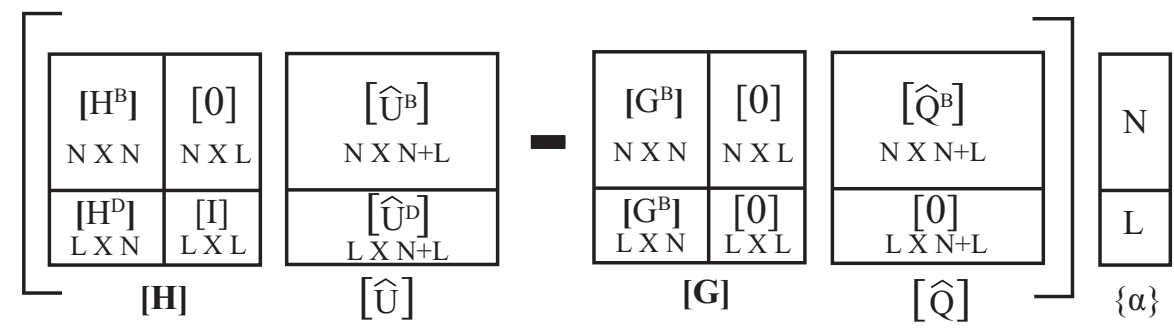

Figure 4.2: DRM Matrices

The right-hand side of Equation (4.22) can be completely expressed in terms of known values by replacing $\{\alpha\}$ with Equation (4.19).

$$
[H]\{\phi\}-[G]\left\{\frac{\partial \phi}{\partial \mathbf{n}}\right\}=[[H][\hat{U}]-[G][\hat{Q}]][F]^{-1}\{b\}
$$




\subsubsection{Complex RHS}

Now that the groundwork has been established for applying the DRM to solve problems with simple Poisson terms, the formulation can now be extended to solve problems with a more complex RHS. These problems can include a Poisson term that is a linear or nonlinear function of the solution. This section will focus specifically on solving problems with a right-hand side that is nonlinear since the full potential equation is also nonlinear.

The numerical implementation of the method will be explained by solving an example problem that can be found in the literature. $[23,19,20]$ Burger's equation in two dimensions is a non-linear Poisson equation because the RHS is a function of the solution and its derivative.

$$
\nabla^{2} \phi=-\phi \frac{\partial \phi}{\partial x}=b
$$

This problem will be solved on an enclosed elliptical domain centered at $(3.0,0.0)$ with a major axis of 2 and a minor axis of 1 . The boundary is discretized with 40 panels and 17 internal points as shown in Figure 4.3. The placement of the internal nodes follows from the example problem found in the literature. The problem has the following analytical solutions:

$$
\begin{gathered}
\phi=\frac{2}{x} \\
\frac{\partial \phi}{\partial \mathbf{n}}=\frac{-2}{x^{2}} n_{x}
\end{gathered}
$$

The derivative term in the RHS of Equation (4.26) can be approximated using radial 


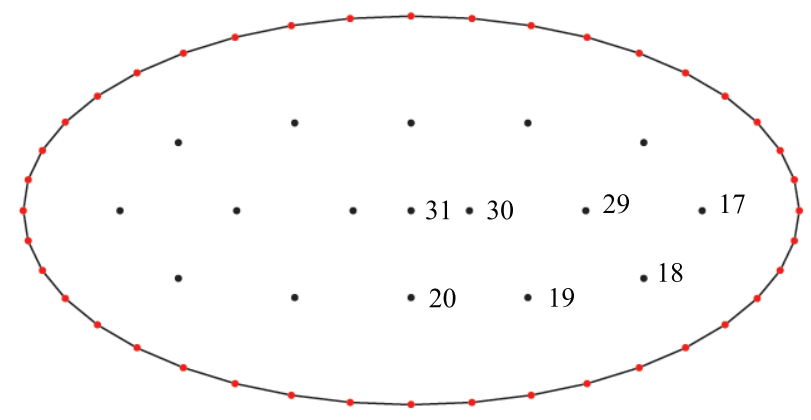

Figure 4.3: Ellipse Geometry

basis functions. Consider an arbitrary scalar function, $\Psi$, that can be approximated using a radial basis function.[16]

$$
\begin{gathered}
\{\Psi\} \cong[F]\{\beta\} \\
\{\beta\}=[F]^{-1}\{\Psi\}
\end{gathered}
$$

If the derivative of the radial basis function can be evaluated, the derivative of $\Psi$ is simply the matrix consisting of the derivatives of the radial basis function, $\left[F_{x}\right]$, multiplied by the vector $\{\beta\}$.

$$
\left[\frac{\partial \Psi}{\partial x}\right] \cong\left[\frac{\partial F}{\partial x}\right]\{\beta\}=\left[\frac{\partial F}{\partial x}\right][F]^{-1}\{\Psi\}=\left[F_{x}\right][F]^{-1}\{\Psi\}
$$

The RBF $f=1+r^{3}$ was chosen for this example because it has a non-singular derivative.

$$
\frac{\partial f}{\partial x}=3 x r
$$

The Poisson term in the discretized form of (4.26) can now be expressed as a function of RBF's.

$$
\left\{\nabla^{2} \phi\right\} \cong-[\phi]\left[F_{x}\right][F]^{-1}\{\phi\} \cong\{b\}
$$


The matrix $[\phi]$ is a $[N+L \times N+L]$ diagonal matrix with the diagonal entries containing the values of $\phi$ at all the $N+L$ points. The $\{\phi\}$ term is a $\{N+L \times 1\}$ vector containing the value of $\phi$ at all the $N+L$ points.

$$
\begin{gathered}
{[\phi]=\left[\begin{array}{cccc}
\phi_{1} & 0 & \cdots & 0 \\
0 & \phi_{2} & \cdots & 0 \\
\vdots & \vdots & \ddots & 0 \\
0 & 0 & \cdots & \phi_{N+L}
\end{array}\right]} \\
\{\phi\}=\left[\phi_{1} \cdots \phi_{N+L}\right]^{T}
\end{gathered}
$$

A vector $\{R\}$ is now defined that contains all the terms in the right hand side of Equation (4.22).

$$
\{R\}=[[H][\hat{U}]-[G][\hat{Q}]]\{\alpha\}
$$

In the previous section this term would have been a known function because the Poisson term is decoupled from the solution variable. For Burger's equation this term is now a function of $\phi$. Therefore the $\alpha$ vector in Equation (4.36) is now defined as:

$$
\{\alpha\}=-[F]^{-1}[\phi]\left[F_{x}\right][F]^{-1}\{\phi\}
$$

The problem is setup so that the Dirichlet boundary condition is known. This results in a mixed type of unknowns: $\left\{\frac{\partial \phi}{\partial \mathbf{n}}\right\}$ on the boundary and $\left\{\phi^{D}\right\}$ in the domain. Therefore Equation (4.22) needs to be modified to account for the mixed type of unknowns. This can be easily done by defining a new system of equations which is a re-arrangement of the terms in Equation (4.22).

$$
[A]\{x\}=[B]\{y\}+\{R\}
$$


The matrix $[A]$ is a $[N+L \times N+L]$ matrix with entries associated with the problem unknowns. The vector $\{x\}$ contains the problem unknowns.

$$
\begin{gathered}
{[A]=\left[\begin{array}{cc}
-G_{1 \ldots N+L, 1 \ldots N} & H_{1 \ldots N+L, N+1 \ldots N+L}
\end{array}\right]} \\
\{x\}=\left[\frac{\partial \phi}{\partial n} \phi^{D}\right]^{T}
\end{gathered}
$$

The matrix $[B]$ is a $[N+L \times N+L]$ matrix with entries associated with the problem knowns. The vector $\{y\}$ contains the problem knowns.

$$
\begin{gathered}
{[B]=\left[\begin{array}{c}
-H_{1 \ldots N+L, 1 \ldots N} \\
G_{1 \ldots N+L, N+1 \ldots N+L}
\end{array}\right]} \\
\{y\}=\left[\begin{array}{ll}
\phi^{B} & 0
\end{array}\right]^{T}
\end{gathered}
$$

Once these matrices are assembled the system can be solved.

$$
\{x\}=[A]^{-1}[[B]\{y\}+\{R\}]
$$

Since the calculation of the vector $\{\alpha\}$ involves the unknown variable $\left\{\phi^{D}\right\}$, an iterative procedure needs to be developed in order to correctly solve the problem. The iterative procedure is illustrated in Figure 4.4.

The convergence criteria was chosen to be the maximum error between the $\left\{\phi^{D}\right\}$ vector at the current iteration step and the previous iteration step. The convergence tolerance was set at $10^{-6}$ which was obtained in 10 iterations without a relaxation parameter. A relatively predictable convergence history is shown in Figure 4.5.

$$
\epsilon=\max \left(\left|\left\{\phi^{D(n+1)}\right\}-\left\{\phi^{D(n)}\right\}\right|\right)
$$




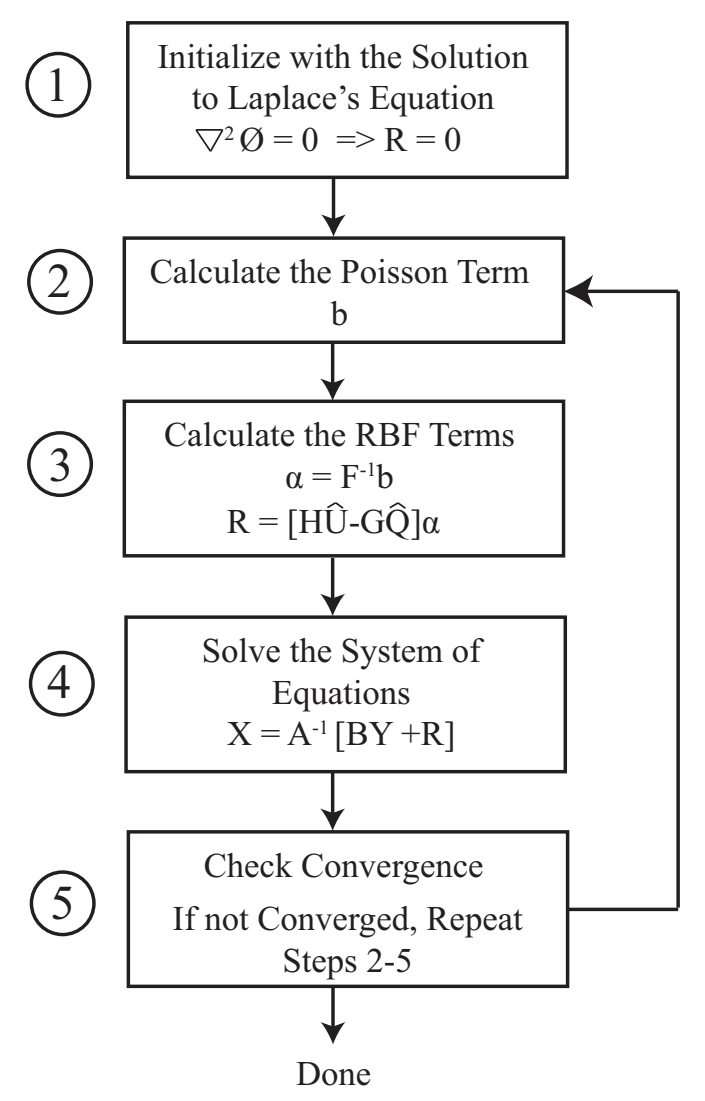

Figure 4.4: Iterative Procedure

Values of $\phi$ at the set of nodes labeled in Figure 4.3 were compared to the analytical solution. The good agreement with the analytical solution along with the rapid convergence suggests that the Dual Reciprocity Method is well suited for non-linear problems. The theory and implementation of the DRM presented in this section will be carried over into the next section which discusses how to solve the full potential equation. 


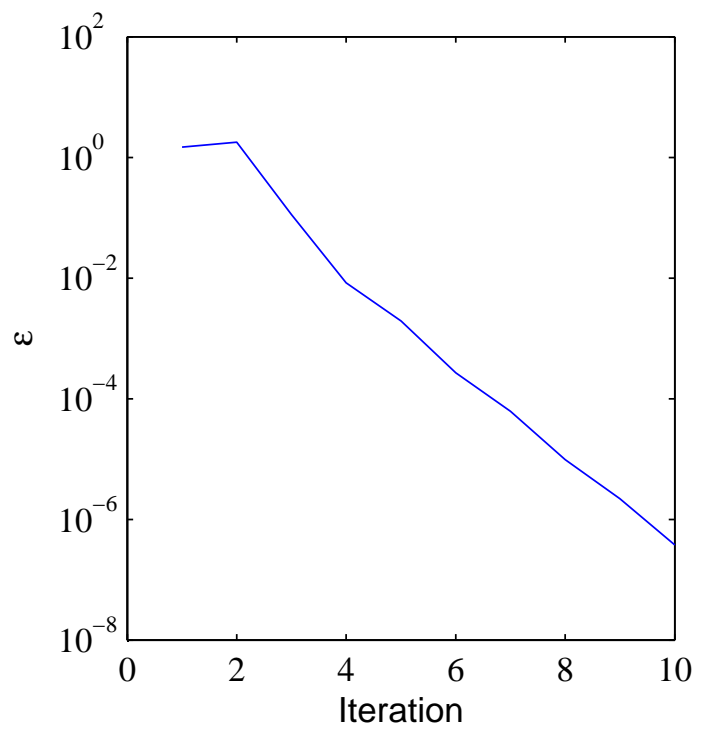

Figure 4.5: Iteration History

Table 4.1: Domain Node Results

\begin{tabular}{ccccc}
\hline \hline Node & $\mathrm{x}$ & $\mathrm{y}$ & Numerical & Exact \\
\hline 17 & 4.5 & 0.0 & 0.449 & 0.444 \\
18 & 4.2 & -0.35 & 0.479 & 0.476 \\
19 & 3.6 & -0.45 & 0.557 & 0.556 \\
20 & 3.0 & -0.45 & 0.668 & 0.667 \\
29 & 3.9 & 0.0 & 0.515 & 0.513 \\
30 & 3.3 & 0.0 & 0.608 & 0.606 \\
31 & 3.0 & 0.0 & 0.668 & 0.667
\end{tabular}

\subsection{Formulation for Compressible Flows}

Chapter 3 described how panel methods can solve an incompressible and irrotational flow problem and Chapter 2 showed how the PDE governing potential flows changes from a Laplace to a Poisson-type equation called the full potential equation which describes steady, irrotational, and isentropic flows ranging from subsonic to supersonic Mach numbers. [3] This thesis will focus on solving the full potential equa- 
tion for subcritical compressible flows so that the PDE stays elliptic throughout the entire flowfield.

\subsubsection{DRM Solution Procedure}

For now, the flow will be assumed to have no lift; therefore, no wake surface will be modeled. Since the PDE is a nonlinear function of the potential, the procedure for solving the equation using the DRM will be iterative. The solution process follows the same basic steps depicted in Figure 4.4. This section will discuss how to execute each of these steps.

\section{Step 1: Initialize with the Solution to Laplace's Equation}

The solution to Laplace's equation is simply the panel method solution described in the previous chapter. Equation (3.39) is solved to determine the value of the perturbation potential at the boundary and domain points.

\section{Step 2: Calculate the Poisson Term}

The velocities need to be calculated first because the density and Poisson term is a function of the velocity field. The velocities on the boundary are calculated using the same finite difference scheme discussed in the previous chapter. Equations (3.37) and (3.38) are used to calculate the velocities in the domain for the first iteration step. For subsequent iterations, the domain velocities are calculated using a mixture of boundary integrals and radial basis functions.

The boundary integral equations used for the incompressible velocities can be 
extended for the non-linear flow by calculating the gradient of Equation (4.25).

$$
\begin{aligned}
\left\{\frac{\partial \phi^{D}}{\partial x}\right\}= & {\left[\left[\frac{\partial \hat{U}^{D}}{\partial x}\right]+\left[\overline{\mathbf{H}}_{u}\right]\left[\hat{U}^{B}\right]-\left[\mathbf{G}_{u}\right]\left[\hat{Q}^{B}\right]\right]\{\alpha\}-} \\
& {\left[\left[\overline{\mathbf{H}}_{u}\right]\left\{\phi^{B}\right\}-\left[\mathbf{G}_{u}\right]\left\{\frac{\partial \phi}{\partial \mathbf{n}}\right\}\right] } \\
\left\{\frac{\partial \phi^{D}}{\partial y}\right\}= & {\left[\left[\frac{\partial \hat{U}^{D}}{\partial y}\right]+\left[\overline{\mathbf{H}}_{v}\right]\left[\hat{U}^{B}\right]-\left[\mathbf{G}_{v}\right]\left[\hat{Q}^{B}\right]\right]\{\alpha\}-} \\
& {\left[\left[\overline{\mathbf{H}}_{v}\right]\left\{\phi^{B}\right\}-\left[\mathbf{G}_{v}\right]\left\{\frac{\partial \phi}{\partial \mathbf{n}}\right\}\right] }
\end{aligned}
$$

where $\left[\frac{\partial \hat{U}}{\partial x}\right]$ and $\left[\frac{\partial \hat{U}}{\partial y}\right]$ are $[L \times N+L]$ matrices composed of the derivatives of the particular solution $\hat{u}$. The components of the total velocity are:

$$
\begin{aligned}
& \left\{U^{D}\right\}=\left\{\frac{\partial \phi^{D}}{\partial x}\right\}+\left\{U_{\infty}\right\} \\
& \left\{V^{D}\right\}=\left\{\frac{\partial \phi^{D}}{\partial y}\right\}+\left\{V_{\infty}\right\}
\end{aligned}
$$

Once the velocities are determined they can be substituted into Equation (2.5) to calculate the density ratio. With both the velocities and density ratio known, the Poisson term $\left\{b^{n+1}\right\}$ can now be evaluated. Different procedures exist depending on whether the conservative or non-conservative form of the equation is being used. For the conservative form of the equation, the quantities associated with the conservative form of the full potential equation, $\left\{m_{u}\right\}$ and $\left\{m_{v}\right\}$, need to be first calculated which contain the values of $(1-\rho) \nabla \vec{U}$ at each of the $i=1 \ldots N+L$ points.[16]

$$
\begin{aligned}
& \left\{m_{u}\right\}=\{1-\rho\}\{U\} \\
& \left\{m_{v}\right\}=\{1-\rho\}\{V\}
\end{aligned}
$$




$$
\{b\}^{n+1}=\left[F_{x}\right][F]^{-1}\left\{m_{u}\right\}+\left[F_{y}\right][F]^{-1}\left\{m_{v}\right\}
$$

The non-conservative form of the Poisson term is determined by directly taking the gradient of the density.

$$
b_{i}^{n+1}=-\frac{1}{\rho_{i}^{n}}\left[\left(\left[F_{x}\right][F]^{-1} \rho^{n}\right)_{i} U_{i}^{n}+\left(\left[F_{y}\right][F]^{-1} \rho^{n}\right)_{i} V_{i}^{n}\right]
$$

\section{Step 3: Calculate the RBF Terms}

This step is kept un-modified from the procedure described for Burger's Equation. The RBF coefficients are found by applying Equation (4.37) and $\{R\}$ is found by calculating Equation (4.36).

\section{Step 4: Solve the System of Equations}

Unlike the Burger's equation solution where $\phi$ was known on the boundary, the Neumman boundary condition is known for compressible flows. It is simply the solid wall boundary condition from Equation (2.13). This means that the matrices and vectors from Equation (4.38) are:

$$
\begin{gathered}
{[A]=[H]} \\
\{x\}=\{\phi\} \\
{[B]=[G]} \\
\{y\}=\left\{\frac{\partial \phi}{\partial \mathbf{n}}\right\}=\left\{\begin{array}{r}
-\frac{\partial \Phi_{\infty}}{\partial \mathbf{n}} \mathrm{i}=1 \ldots \mathrm{N} \\
0 \mathrm{i}=\mathrm{N}+1 \ldots \mathrm{N}+\mathrm{L}
\end{array}\right.
\end{gathered}
$$

So far the general procedure used here has mirrored the methods used by Theurer, but with one exception. Theurer presents the dual reciprocity method as if the terms 
on the left hand side of Equation (4.25) are only associated with the linear part of the perturbation potential, $\tilde{\phi}$, instead of the total perturbation potential, $\phi$. If this assumption is true then, the boundary condition on $\tilde{\phi}$ would be

$$
\frac{\partial \tilde{\phi}}{\partial \mathbf{n}}=-\left(\frac{\partial \Phi_{\infty}}{\partial \mathbf{n}}+\frac{\partial \hat{\phi}}{\partial \mathbf{n}}\right)
$$

As a result, Theurer adds an extra step where the incompressible boundary condition is modified to account for the effects of compressibility. Instead of $\{y\}$ being defined as it was above, Theurer adds the effect of $\left\{\frac{\partial \hat{\phi}}{\partial \mathbf{n}}\right\} \cdot[16,7]$

$$
\{y\}=-\left(\left\{\frac{\partial \Phi_{\infty}}{\partial \mathbf{n}}\right\}+\left\{\frac{\partial \hat{\phi}}{\partial \mathbf{n}}\right\}\right)
$$

The results in the next section will show that the formulation presented in this section is capable of representing the flow physics even though it is in conflict with the presentation made by Theurer et. al.

\section{Step 5: Check Convergence}

The convergence criteria for this problem will use the error estimator used by Theurer.[16] The iteration error is defined as the maximum difference between the Poisson term at the most recent time step and the previous time step. Until this value reaches a desired tolerance level, steps $2-5$ will be repeated.

$$
\epsilon_{b}=\max \left(\left|\{b\}^{n+1}-\{b\}^{n}\right|\right)
$$

\subsubsection{RBF Selection Criteria}

Before some numerical cases can be examined, it is important to note that there are added restrictions on the choice of RBF's used for modeling problems with an 
infinite domain. Theurer et. al. circumvented this issue by directly modeling the farfield boundary as a discretized circle that was sufficiently far away from the airfoil surface.[16] This thesis will focus on how to implement the DRM for infinite domains without needing to discretize the farfield boundary.

As discussed in Section 3.1.1, certain regularity conditions need to be satisfied in order for an external problem to be solved. It was shown that the behavior of the potential and the Green's function at a point infinitely far away from the airfoil satisfy the regularity conditions. If the incompressible solution satisfies the regularity conditions, it would follow that the non-linear solution would also need to satisfy these conditions.

A literature search resulted in a limited amount of papers discussing how to implement the Dual Reciprocity Method for infinite domain problems.[24, 25] The first article that discussed the implementation of the DRM for infinite problems was written by Loeffler and Mansur.[24] They used the DRM to solve transient heat conduction problems. When trying to solve the case of heat flow near a hole in an infinite plate, they showed that the RBF's used to represent the particular solutions also needed to have a form that would satisfy the regularity conditions.

They suggested estimating the particular solutions with the following RBF's:

$$
\begin{gathered}
f=\frac{2 c-r}{(r+c)^{4}} \\
\hat{u}=\frac{c+2 r}{2(r+c)^{2}}
\end{gathered}
$$




$$
\begin{gathered}
\hat{q}=\frac{r}{(r+c)^{3}} \frac{\partial r}{\partial n}=\frac{\left(x n_{x}+y n_{y}\right)}{(r+c)^{3}} \\
\frac{\partial \hat{u}}{\partial x}=\frac{x}{(r+c)^{2}} \\
\frac{\partial \hat{u}}{\partial y}=\frac{y}{(r+c)^{2}}
\end{gathered}
$$

where $c$ is an adjustable parameter that the authors provided an empirical estimate for the transient heat conduction problem. It can be shown that these RBF's exhibit the order of decay necessary for the regularity condition to be satisfied.

$$
\begin{aligned}
& \hat{u}=O\left(r^{-1}\right) \\
& \hat{q}=O\left(r^{-2}\right)
\end{aligned}
$$

Zhu and Zhang [25] pointed out that the empirical estimate provided by Loeffler and Mansur gave a lower bound on the parameter $c$ but no upper bound. Zhu and Zhang observed that as the value of $c$ increased the matrix $[F]$ would become nearly singular. The authors showed for a trial problem that there is a problem dependent optimal value of $c$ for which the average error between the DRM solution and the analytical solution would be minimized. They postulated that the optimal $c$ value is a compromise between making $f_{i k}$ nearly singular when $i=k$ for a small $c$ value and the matrix $[F]$ being nearly singular for too large a value of $c$. The next section will show some numerical results for compressible flow problems with these numerical considerations taken into account.

It is important to note that while these RBF's are integrable, their derivatives are 
singular when $r=0$.

$$
\begin{aligned}
& \frac{\partial f}{\partial x}=\frac{\partial f}{\partial r} \frac{\partial x}{\partial r}=\frac{3 r-9 c}{(r+c)^{5}} \frac{x}{r}=\frac{\left(3 x-\frac{9 c x}{r}\right)}{(r+c)^{5}} \\
& \frac{\partial f}{\partial y}=\frac{\partial f}{\partial r} \frac{\partial y}{\partial r}=\frac{3 r-9 c}{(r+c)^{5}} \frac{y}{r}=\frac{\left(3 y-\frac{9 c y}{r}\right)}{(r+c)^{5}}
\end{aligned}
$$

Therefore the RBF suggested by Loeffler and Mansur will be used to estimate the particular solution, but the $\operatorname{RBF} f=1+r^{3}$ will be used to determine the gradient of density in the subsequent validation cases. The mixing of the two different RBF's would suggest that problems with consistency may be encountered because one interpolant is being used for integration of the potential whereas a different interpolant is used for the derivative of field variables. The subsequent verification problems will show that if this consistency issue exists, its influence on the accuracy of the solution is minimal.

\subsection{Results}

\subsubsection{Cylinder Problem}

The first validation example is the analysis of compressible flow over a cylinder. The freestream flow will have a Mach number $M_{\infty}=0.375$ and a ratio of specific heats $\gamma=1.4$. The pressure coefficient can be calculated as follows.

$$
C_{p}=\frac{2}{\gamma M_{\infty}^{2}}\left\{\left[1+\frac{\gamma-1}{2} M_{\infty}^{2}\left(1-|\nabla \Phi|^{2}\right)\right]^{\frac{\gamma}{\gamma-1}}-1\right\}
$$

An analytical solution for the peak velocity on a circular cylinder in compressible potential flow was developed by Van Dyke [26] using a Janzen-Rayleigh series 
expansion. This expansion involves a set of 29 terms and integer powers of $M_{\infty}{ }^{2}$.

$$
V_{\text {max }}=U_{\infty} \sum_{n=1}^{29} q_{n}\left(M_{\infty}^{2}\right)^{n-1}
$$

Since this analytical solution only allows for a comparison at the velocity peak, results from the Ph. D. dissertation written by German [27] was also used because it provides results for the entire distribution of $C_{p}$ on the circle surface. German developed a Riemannian geometric mapping technique to determine the compressible flowfield by solving a panel method on an equivalent incompressible shape.[28, 29] The method was extensively verified and showed that the peak velocity closely matched the analytical result determined by Van Dyke.

The solution procedure developed by German involved the generation of an Ogrid around the cylinder surface. The initial set of domain nodes for the DRM will use a subset of the nodes in the grid. The grid consisted of a $[121 \times 121](r, \theta)$ grid with uniformly spaced nodes in the angular $(\theta)$ direction and 121 nodes exponentially distributed in the radial $(r)$ direction. The details on the exponential distribution function can be found in Reference [27]. The spacing parameter, $\zeta$ was chosen to be 0.01 which corresponds to an almost linear distribution of nodes in the radial direction. A closeup view of the grid is shown in Figure 4.6. The outer boundary of the grid extends radially to 2.5 chord lengths away from the center located at $(0.5$, $0)$.

In order to determine how far away from the circle the surface nodes need to be distributed for the DRM solution, the Poisson term was calculated on the grid using 


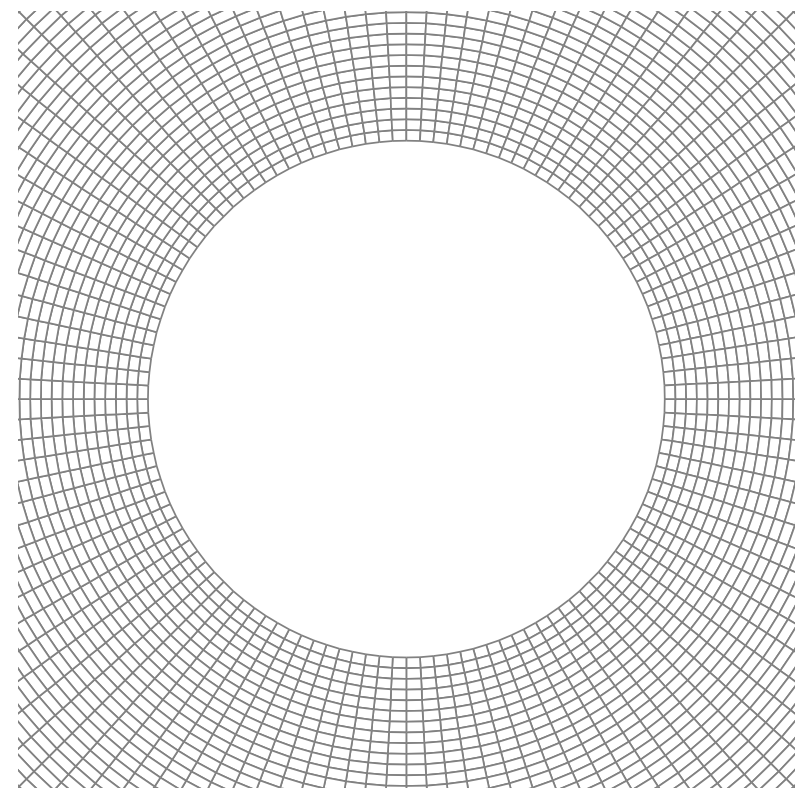

Figure 4.6: Cylinder O-Grid Near Body

the velocities obtained from German's converged solution. It was observed that here was not much difference between calculating the Poisson term in its conservative or non-conservative form. Contours of the non-conservative Poisson term are shown in Figure 4.6. The nonlinearity of the flow is mostly restricted to an area encompassed by a radius of one chord length from the cylinder center. Therefore the domain points used for the calculation of the DRM solution were chosen not to extend past this region.

Within this region there exists 2880 unique domain points. If the circle surface was discretized by 60 panels, the total number of $N+L$ nodes would be 2940 nodes. This amount of nodes was infeasible because of the requirement to store several 


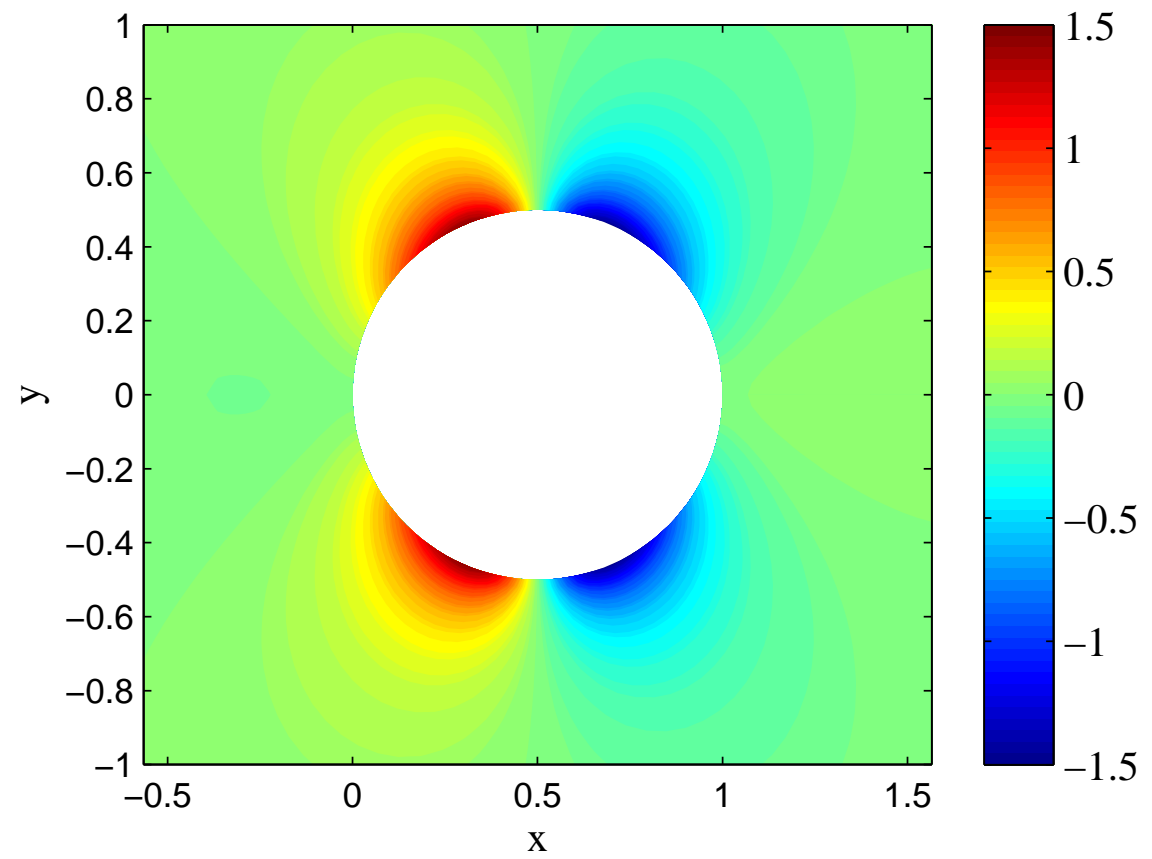

Figure 4.7: Non-Conservative Cylinder Poisson Term

$[N+L \times N+L]$ matrices that need to be assembled and inverted for the DRM procedure which would exceed the memory capacity of the computer. Therefore, a reduced set of the 2880 domain points was used. If every other value of $\theta$ for rings of constant $r$ and then every other value of $r$ for lines of constant $\theta$ were thrown out, the number of domain points would be reduced to $L=720$. This amount of domain points is depicted in Figure 4.8.

There are several sources that provide estimates for what the parameter $c$ should 


\section{- Domain Point}

Circle Surface

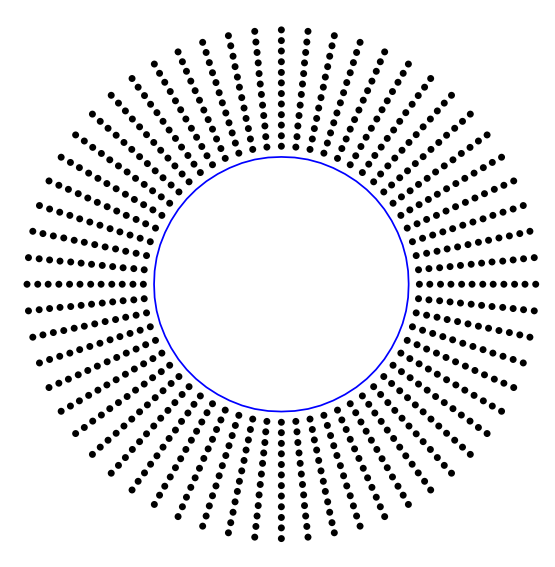

Figure 4.8: Cylinder Domain Points

be with respect to another type of radial basis function known as the multiquadric RBF. $[30,31,32,33,34,35,36,37,38,39,40]$. These sources use either empirical estimates or some other technique to determine what value of $c$ will minimize a predefined error estimator. In either case the selection of the most accurate parameter uses a priori knowledge of the solution.

The best value of $c$ for the cylinder problem was determined by simply solving the system with varying values of $c$ and then calculating the normalized error between the calculated suction peak velocity and the analytical solution from Van Dyke. As 
shown in the Figure 4.9, the error is minimized when $c \cong 1.46$

$$
\epsilon=\left|\frac{V_{a}-V_{e}}{V_{e}}\right|
$$

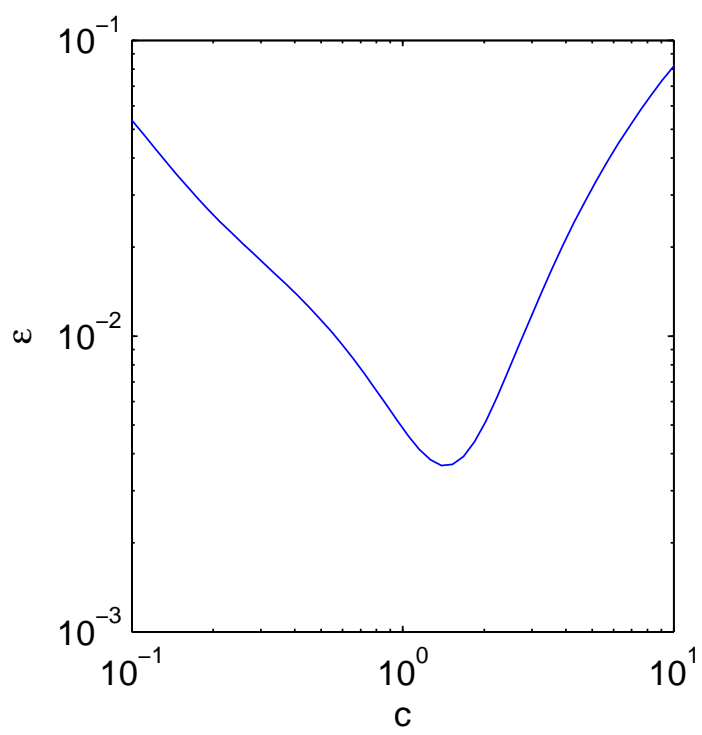

Figure 4.9: Suction Peak Error

Figures 4.10 and 4.11 depict the results from running the DRM solution with the optimized value of $c$. The iteration history shows that the solution converges quite rapidly and in a smooth and predictable manner. The matrix inversions necessary for computing the RBF coefficients and for evaluating fluxes only need to be calculated once and then stored; therefore the iterative process is quite fast since only matrix multiplication and addition is required to update the solution. As can be seen in Figure 4.11, the pressure coefficient distribution matches quite well with the results from German and the suction peak velocity error was calculated to be $3.6 \times 10^{-3}$. 
Therefore the optimized value of $c$ resulted in an accurate local and global prediction of the flowfield.

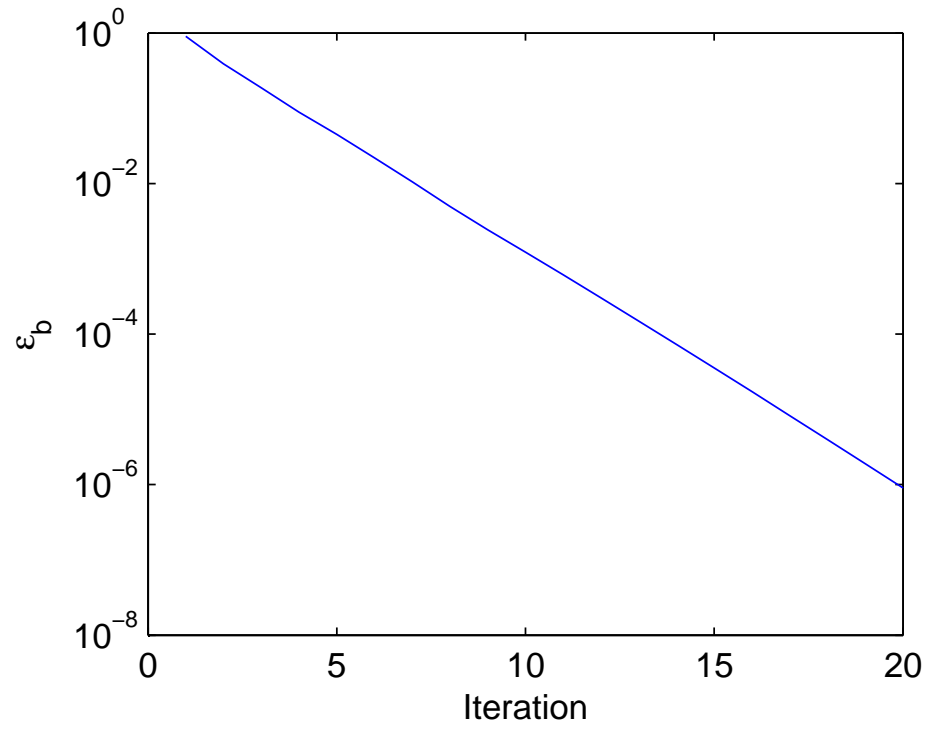

Figure 4.10: Cylinder Iteration History

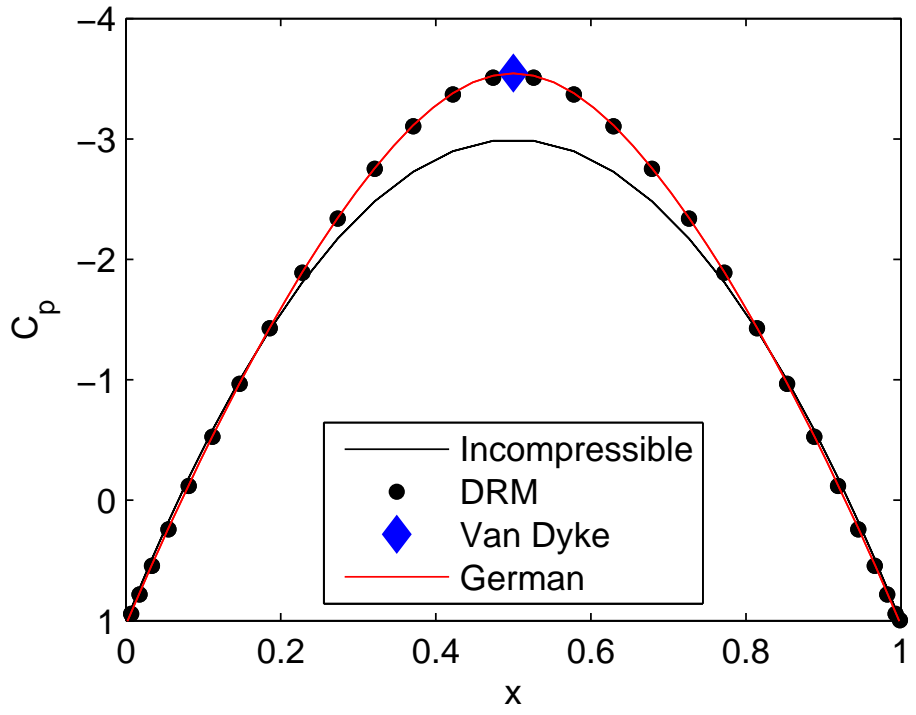

Figure 4.11: Cylinder Pressure Coefficient Distribution $\left(M_{\infty}=0.375\right)$ 


\subsubsection{Airfoil Problem}

In order to assess the capability to model flows of engineering interest, a NACA 0012 airfoil with a freestream Mach number of 0.72 at a zero angle of attack was analyzed. German also analyzed this case by comparing results to an AGARD report written by Lock.[41] This report is considered a benchmark case since Lock notes that the results should be within one percent of the exact full potential solution.

German generated a grid using the same procedure for the cylinder. The Poissonterm was calculated from German's converged solution and the results are shown below. The region where compressibility effects are present is smaller when compared to the results for the cylinder from the previous section, but the magnitude of the Poisson term is a higher value.

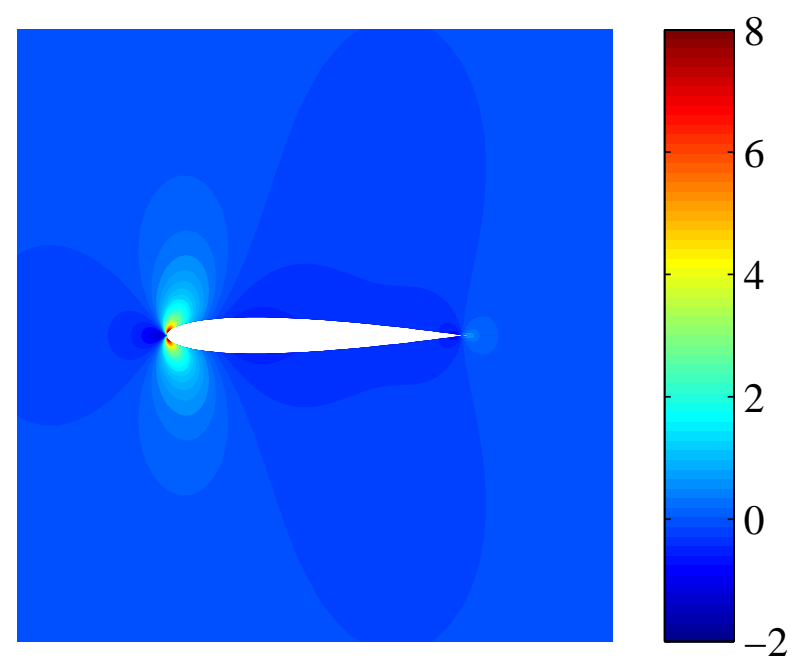

Figure 4.12: NACA 0012 Poisson Term

A reduced set of the grid points used by German was chosen for the dual reciprocity 


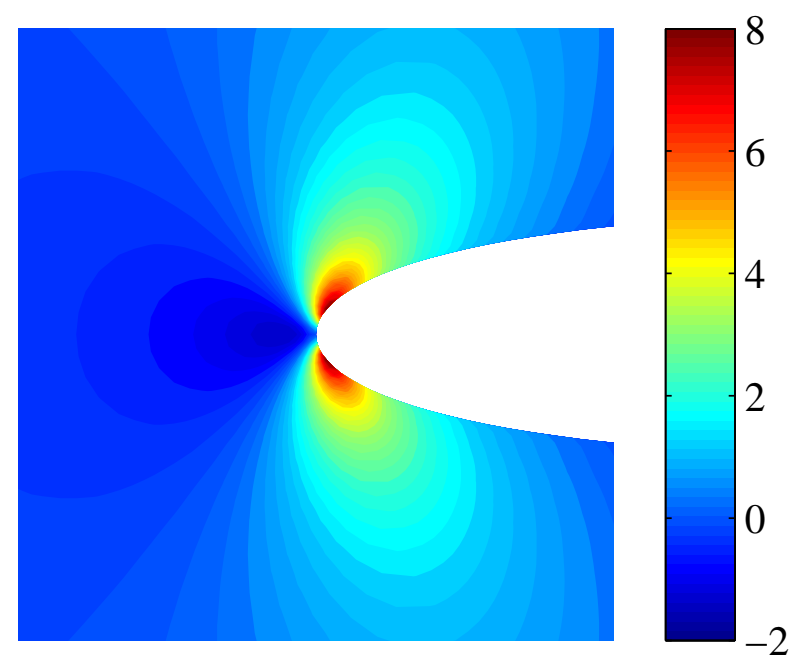

Figure 4.13: Poison Tern Near Leading Edge

solution. The points depicted below encompass an area that captures the majority of the compressibility effects. The reduced set provides a total of $L=1200$ domain points and $N=80$ panels are used to discretize the surface.

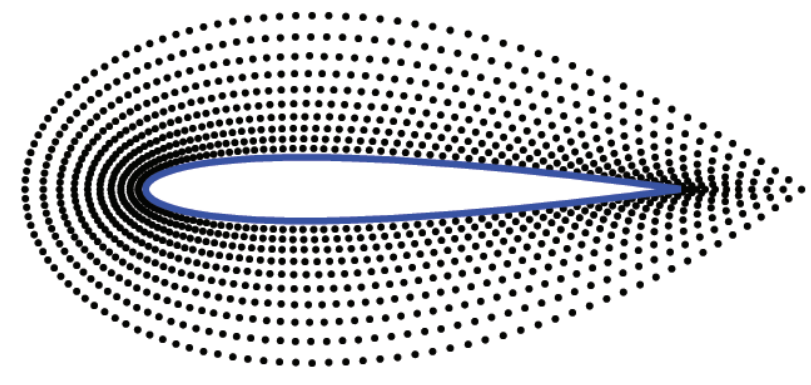

Figure 4.14: Domain Points

The $c$ parameter was chosen in an ad-hoc manner by testing a range of values and comparing the resulting pressure coefficient distributions to the data from Lock. In all of the cases the largest amount of error was present in the pressure recovery region just aft of the suction peak. The results below are for a $c$ parameter equal 
to 0.35 which gave the best compromise between the error in the pressure recovery region and the error in capturing the magnitude and location of the suction peak. The iteration history shows that just like the cylinder problem, the convergence is well behaved.

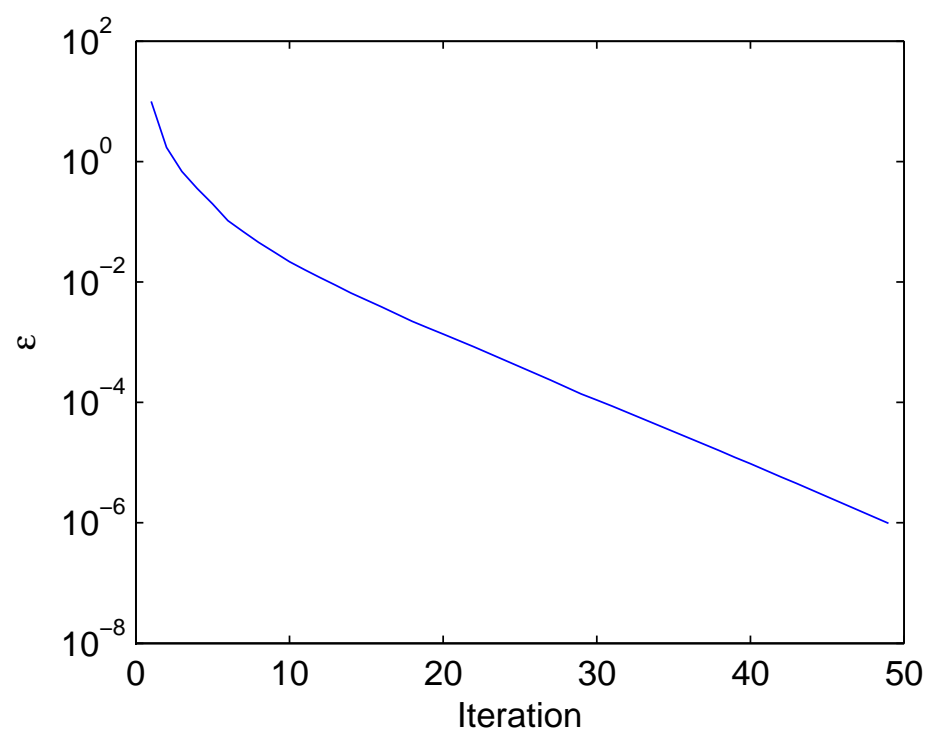

Figure 4.15: NACA 0012 Iteration History

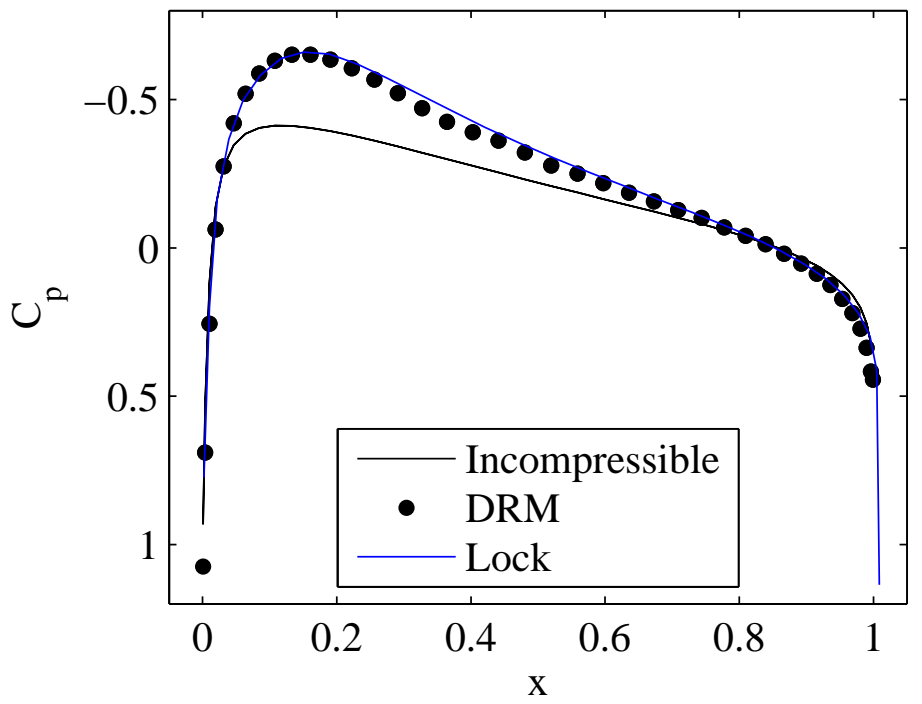

Figure 4.16: NACA 0012 Cp Distribution $\left(\alpha=0, M_{\infty}=0.72\right)$ 
In order to achieve an accurate solution, a priori knowledge of the solution was required to determine the region where domain nodes are needed and for the correct value of the $c$ parameter. While there is a vast amount of literature on the use of the Dual Reciprocity Method for problems with bounded domains, there is a limited amount on the subject of unbounded domains. The implementation of the DRM utilized by Theurer et. al. explicitly modeled the farfield boundary which has the potential to require a large amount of domain points that would have to extend to the farfield. The method employed here only required the placement of domain nodes where compressiblity is expected to be dominant. Therefore the number of nodes necessary for convergence is smaller since compressibility effects are confined to regions close to the geometry surface. While the results showed that the DRM is capable of solving problems of engineering interest, the fact that the $c$ parameter needs to be "tuned" to achieve an accurate solution suggests that this method is not a robust design tool. Therefore the next chapter will be devoted to developing an accurate meshless method that does not require previous knowledge about the solution. 


\section{Chapter 5}

\section{The Generalized Finite Difference Method}

The limitations associated with the Dual Reciprocity Method led to the search for a different meshless method that could solve the full potential equation. This search resulted in the discovery of methods that could solve a PDE in differential form rather than integral form. These solution techniques are most widely known as meshless methods and can be best described as techniques that do not employ grid data to discretize a partial differential equation. There are a multitude of different algorithms within this research area that have been formulated to solve a vast array of different engineering problems. A comprehensive review of the most popular meshless methods can be found in the book written by Liu[42] and the review paper written by Nguyen.[43].

A subset of solution techniques within this broad research area are those that apply the concept of discretizing a PDE on a local cloud of points. Unlike the RBF interpolation employed in the previous chapter, each of the points in the global set 
have their own local cloud with associated support points. The cloud shown in Figure 5.1 can either be selected by having it encompass a constant local area or contain a pre-determined amount of points. In either case, this concept is not a relatively new one [44], yet its implementation to solve aerodynamic problems has only been an active research topic in the last two decades.[45]

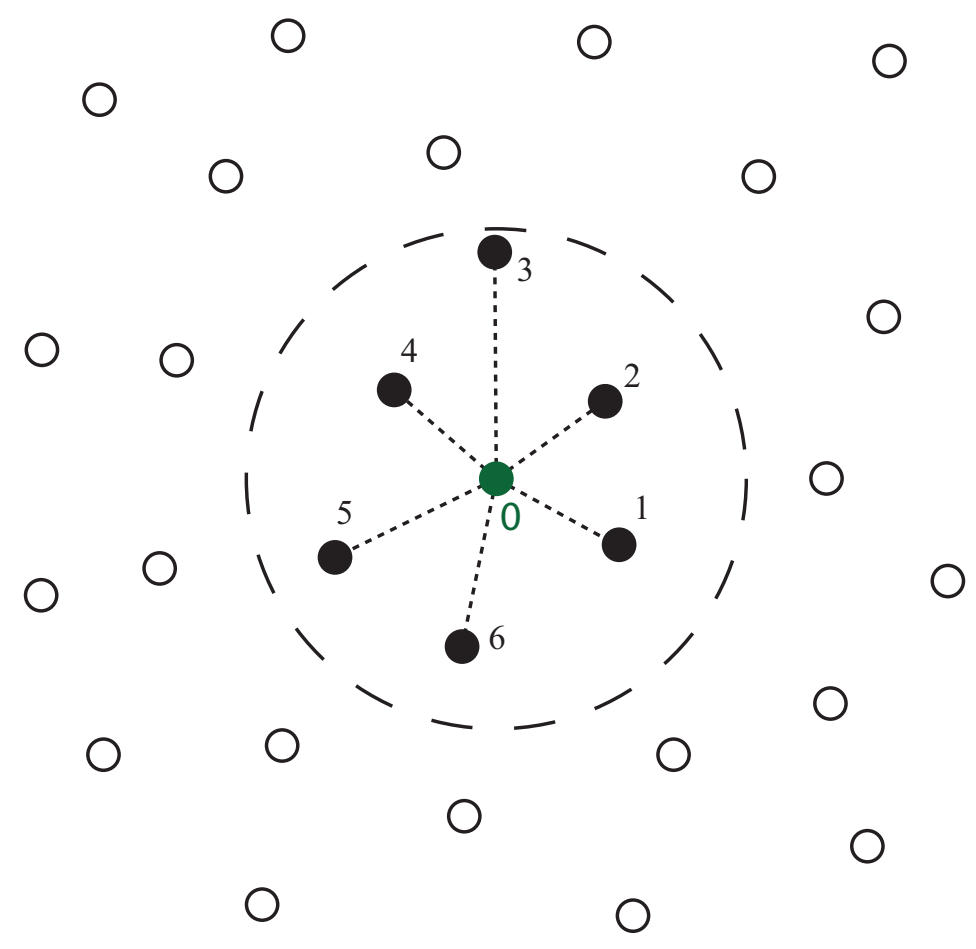

Figure 5.1: Point Cloud

Methods that apply the local cloud concept have been given a variety of names within the literature such as finite point mehtods [46], the generalized finite difference method [47], or local RBF differential quadrature [48]. The finite point method refers to applying a least squares fit of an approximating function on the local cloud of points. This least squares fit can either involve a polynomial basis [46] or a truncated 
Taylor series [49]. The term generalized finite difference method was first coined by Chung in 1981 [47] and it specifically refers to applying a least squares fit of a Taylor series. The local RBF differential quadrature method applies a localized radial basis function interpolation on the points within the cloud. There is also a global cloud method commonly referred to as Kansa's method [31, 32] which applies an RBF interpolation to the entire set of points distributed on the boundary and in the domain. This global method was introduced in 1990 and is the first RBF based technique developed to solve PDE's in differential form.

One of the earliest methods that proposed the discretization of a PDE on a local cloud of points applied the least squares fit of a Taylor series.[50, 49, 51] The earliest meshless method to solve aerodynamic problems by applying the local cloud concept was the work of Batina.[45, 52] The Euler/Navier Stokes solver developed by Batina showed how partial derivatives could be estimated by solving a least squares polynomial fit on a local cloud of points. This method was further developed by Oñate et. al. to solve other fluid mechanic and heat transfer problems of engineering interest. $[46,53,54,55,56]$ Sridar and Balakrishnan revisited the Taylor series concept by developing an upwind meshless Euler solver for two dimensional inviscid transonic flows. $[57,58]$ As mentioned previously, the concept of applying an RBF to solve a PDE in differential form was first proposed by Kansa, but the application of an RBF interpolation on a local cloud of points to solve aerodynamic problems was first developed by Shu to solve the incompressible Navier-Stokes equations.[48] 
The three interpolation techniques (Polynomial, Taylor Series, and RBF) have all been shown to solve aerodynamic problems of engineering interest, but this thesis will focus specifically on the Taylor series approach and it will be referred to as the generalized finite difference (GFD) method. The RBF method was not used because the literature shows that the multiquadric RBF was usually chosen as the interpolation function. While this RBF can produce very accurate derivative estimates, it contains a free parameter $c$ which has to be tuned much like the procedure for the dual reciprocity method; therefore, for the sake of robustness and applicability as a design tool, a method which does not employ ad-hoc parameters such as the $c$ term was sought out. The polynomial method has many similarities to the Taylor series method but an estimate of its order of accuracy has not been formalized as it was for the Taylor series method.[57, 59]

This chapter will be devoted to formulating the Generalized Finite Difference method to solve the full potential equation. The first section will focus on developing the solution algorithm and discuss topics such as how support points are chosen for the cloud and how to discretize the full potential equation into a form that can be solved by the GFD method. The subsequent section will address special implementation considerations that need to be taken into account when solving problems such as lifting airfoils. Finally there will be a discussion in the third section on how to assess the order of accuracy of the schemes employed in this thesis. 


\subsection{Solution Algorithm}

The solution algorithm employed in this chapter works in an analogous manner to a standard finite difference solver. Figure 5.2 outlines the six main steps that the solver written in this thesis performs. This section will discuss in detail how each of these steps are evaluated to solve the full potential equation.

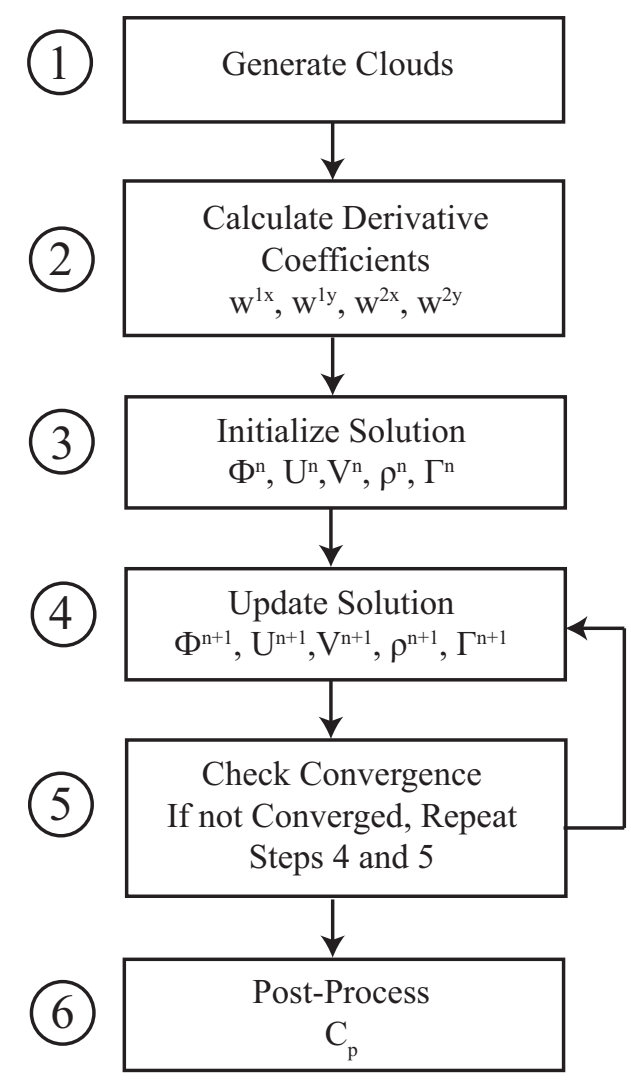

Figure 5.2: Iterative Procedure

\subsubsection{Cloud Generation}

The clouds for all the test cases in this thesis will be constructed from nodes generated by an unstructured mesh generator. While this point generation technique 
would seem to eliminate the claim that this numerical technique is meshless, this thesis is specifically focusing on the solution algorithm which does not depend on how the nodes are initially generated. For this algorithm to be a useful design tool, a point generation technique that does not require meshing of the problem geometry would be essential. Efforts have been made in this area by Löhner and Oñate $[60$, 61] who developed an advancing front point generation technique which shows some promise for the eventual development of a fully integrated meshless point generator and solution algorithm.

As stated earlier, different criteria can be used to determine which points are selected to be a part of the local cloud. This thesis will apply a nearest neighbor search to determine the cloud support points. Each cloud will contain a user specified amount of nearest neighbors instead of selecting clouds of constant area. Figure 5.3 depicts the typical point distributions for a cylinder and NACA 0012 problem. There is a higher density of points near areas where sharp gradients are expected such as the leading and trailing edge of the airfoil. The nearest neighbor search criterion will ensure that the point clouds in regions of higher point density will encompass a smaller area than clouds near the farfield boundary. For the simple two dimensional test cases, this method is quite quick and simple to implement. For problems with a large amount of surface and domain points such as complex three-dimensional configurations, an oct-tree data structure could be implemented to speed up the nearest neighbor search. 


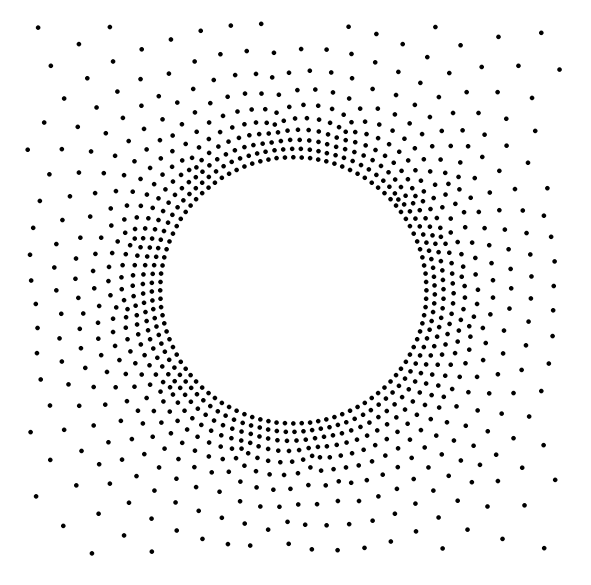

(a) Circle

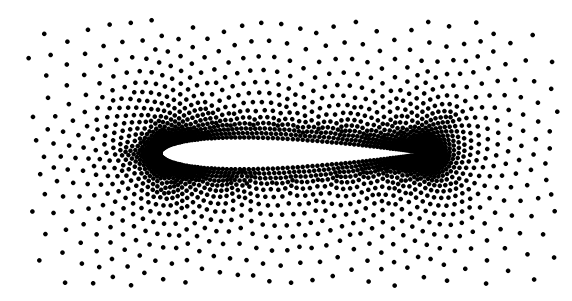

(b) NACA 0012

Figure 5.3: Close-Up View of Point Distributions

\subsubsection{Derivative Coefficient Calculation}

This section will discuss how any order derivative of a function $\phi$ can be discretized into an algebraic sum of its nodal values in the local cloud.

$$
\begin{gathered}
\frac{\partial^{m} \phi_{0}}{\partial d^{m}} \cong \sum_{i=1}^{n s} w_{i}^{m d} \Delta \phi_{i} \\
\Delta \phi_{i}=\phi_{i}-\phi_{0}
\end{gathered}
$$

where $m$ is the order of the derivative, $d$ is the Cartesian direction, and $n s$ is the number of support points in the cloud. For example, the estimate for the second $y$ derivative at node 0 in the cloud shown in Figure $5.1(m=2, d=y, n s=6)$ would be

$$
\frac{\partial^{2} \phi_{0}}{\partial y^{2}} \cong \sum_{i=1}^{6} w_{i}^{2 x} \Delta \phi_{i}
$$

For an arbitrary function $\phi(x, y)$ the truncated Taylor series between the cloud 
center $\phi_{0}=\phi\left(x_{0}, y_{0}\right)$ and the cloud support points $\phi_{i}=\phi\left(x_{i}, y_{i}\right)$ can be expressed in compact form by employing a binomial coefficient.[57]

$$
\Delta \phi_{i}=\sum_{q=1}^{l} \sum_{m=0}^{q}\left(\begin{array}{c}
q \\
m
\end{array}\right) \frac{\Delta x_{i}^{q-m} \Delta y_{i}^{m}}{q !} \frac{\partial^{q} \phi_{0}}{\partial x^{q-m} \partial y^{m}}
$$

where $l$ is the highest order derivative to be calculated in the truncated series and $\Delta \phi_{i}=\phi\left(x_{i}, y_{i}\right)-\phi\left(x_{0}, y_{0}\right), \Delta x_{i}=x_{i}-x_{0}, \Delta y_{i}=y_{i}-y_{0}$. The derivatives of the function can be solved by applying a least squares minimization problem. A weighted residual statement can be made on the Taylor series for the $n s$ points used in the cloud centered at $\left(x_{0}, y_{0}\right)$.

$$
R_{1}=\sum_{i=1}^{n s} w_{i}\left[\Delta \phi_{i}-\sum_{q=1}^{l} \sum_{m=0}^{q}\left(\begin{array}{c}
q \\
m
\end{array}\right) \frac{\Delta x_{i}^{q-m} \Delta y_{i}^{m}}{q !} \frac{\partial^{q} \phi_{0}}{\partial x^{q-m} \partial y^{m}}\right]^{2}
$$

An inverse distance weighting function is used to improve matrix conditioning. [62]

$$
w_{i}=\frac{1}{\left(\Delta x_{i}^{2}+\Delta y_{i}^{2}\right)^{\frac{p}{2}}}
$$

where $p \geq 0$ and was chosen to be 1 . The distances $\Delta x_{i}$ and $\Delta y_{i}$ are also normalized by the maximum distance $\left(r_{\max }\right)$ between the node at $\left(x_{0}, y_{0}\right)$ and $\left(x_{i}, y_{i}\right)$.

$$
r_{\text {max }}=\max \left(\sqrt{\left(x_{i}-x_{)}\right)^{2}+\left(y_{i}-y_{0}\right)^{2}}\right)
$$

Taking the derivative of $R_{1}$ with respect to each of the $l\left(\frac{l+3}{2}\right)$ derivatives and setting each derivative to zero results in a system of equations of the form:

$$
[A][B]\{d\}=[A]\{b\}
$$

The elements within the $[A]$ matrix are generated by determining the combinations of $q$ and $m$ associated with each derivative term. These coefficients can be determined 
in a systematic manner by applying the following algorithm.

$$
k=1
$$

for $q \epsilon 1 \ldots l$ do

for $m \epsilon 0 \ldots q$ do

$$
\begin{aligned}
& q_{k}=q \\
& m_{k}=m \\
& k=k+1
\end{aligned}
$$

\section{end for}

\section{end for}

This algorithm implies that there will be a total of $t=l\left(\frac{l+3}{2}\right)$ different combinations of $q$ and $m$ which will be used to define the coefficients used in each of the rows in the $[A]$ matrix.

$$
[A]=\left[\begin{array}{cccc}
w_{1}\left(\begin{array}{c}
q_{1} \\
m_{1}
\end{array}\right) \frac{\Delta x_{1}^{q_{1}-m_{1}} \Delta y_{1}^{m_{1}}}{q_{1} !} & \cdots & w_{n s}\left(\begin{array}{c}
q_{1} \\
m_{1}
\end{array}\right) \frac{\Delta x_{n s}^{q_{1}-m_{1}} \Delta y_{n s}^{m_{1}}}{q_{1} !} \\
\vdots & \ddots & \vdots \\
w_{1}\left(\begin{array}{c}
q_{t} \\
m_{t}
\end{array}\right) \frac{\Delta x_{1}^{q_{t}-m_{t}} \Delta y_{1}^{m_{t}}}{q_{t} !} & \cdots & w_{n s}\left(\begin{array}{c}
q_{t} \\
m_{t}
\end{array}\right) \frac{\Delta x_{n s}^{q_{t}-m_{t}} \Delta y_{n s}^{m_{t}}}{q_{t} !}
\end{array}\right]
$$

The $[B]$ matrix is simply the transpose of the $[A]$ matrix without the values from the inverse distance weighting function.

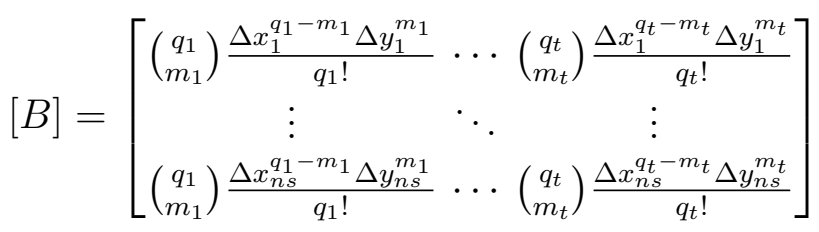

The $\{d\}$ vector contains the $l\left(\frac{l+3}{2}\right)$ partial derivatives of $\phi_{0}$ and the $\{b\}$ vector contains 
the $n s$ differences in function values between $\phi_{0}$ and $\phi_{i}$.

$$
\begin{array}{r}
\{d\}=\left[\begin{array}{c}
\frac{\partial^{q_{1}} \phi_{0}}{\partial x^{q_{1}-m_{1}} \partial y^{m_{1}}} \\
\vdots \\
\frac{\partial^{q_{t}} \phi_{0}}{\partial x^{q_{t}-m_{t}} \partial y^{m_{t}}}
\end{array}\right] \\
\{b\}=\left[\begin{array}{c}
\Delta \phi_{1} \\
\vdots \\
\Delta \phi_{n s}
\end{array}\right]
\end{array}
$$

The derivative coefficients are entries in the $[t \times n s]$ matrix $[T]$ that results from solving the system of equations in Equation (5.8).

$$
\begin{gathered}
{[T]=[[A][B]]^{-1}[A]} \\
\frac{\partial \phi_{0}}{\partial^{q_{k}-m_{k}} x \partial^{m_{k}} y}=\left[t_{k, 1} \cdots t_{k, n s}\right] \times\left[\begin{array}{c}
b_{1} \\
\vdots \\
b_{n s}
\end{array}\right] \\
=\sum_{i=1}^{n s} w^{\left(\left(q_{k}-m_{k}\right) x, m_{k} y\right)} \Delta \phi_{i}
\end{gathered}
$$

As an example, the coefficients associated with calculating the first x-derivative of $\phi$, corresponding to $\left(q_{k}=1, m_{k}=0\right)$, would be the first row of the $[T]$ matrix.

$$
\begin{aligned}
& w^{1 x}=\left[\begin{array}{llll}
t_{1,1} & t_{1,2} & \cdots & t_{1, n s}
\end{array}\right] \\
& \frac{\partial \phi_{0}}{\partial x}=\sum_{i=1}^{n s} w_{i}^{1 x} \Delta \phi_{i}
\end{aligned}
$$

\subsubsection{Solution Initialization}

For all of the test cases in the next chapter the solution will be initialized with the freestream conditions at every point on the boundary and in the domain. Therefore the potential will be $\Phi_{\infty}$, the velocity components are $U=U_{\infty}$ and $V=U_{\infty}$, and the density ratio is $\rho=1$ at every point. 


\subsubsection{Solution Update}

The continuity equation and boundary conditions need to be discretized in order for the PDE to be expressed in an algebraic form which can then be solved for the solution update at the cloud center. Unlike the dual reciprocity method, the total potential will be the solution variable instead of the perturbation component and the farfield boundary will be explicitly modeled.

A Dirichlet boundary condition will be enforced at the farfield boundary which will be modeled as a circle centered at the middle of the airfoil with a radius that is sufficiently far away from the airfoil. The total velocity potential at each point on the outer boundary will be expressed as [9]:

$$
\Phi_{o b}=\Phi_{\infty}+\frac{\Gamma}{2 \pi} \theta
$$

where $\Gamma$ is the circulation strength and $\theta$ is an angle measured from a line extending horizontally from the trailing edge of the airfoil. After all the points have been gone through one iteration, the circulation value is updated by enforcing the Kutta condition in the same manner it was for a panel method by calculating the difference in velocity potential values between the closest points on the upper and lower side of the airfoil trailing edge. The numerical considerations associated with lifting flows will be discussed in further detail in section 5.2.

A Neumann boundary condition is enforced at the airfoil surface. Since the total velocity potential is being determined instead of the perturbation potential this 
boundary condition can be simply expressed as

$$
\frac{\partial \Phi}{\partial \mathbf{n}}=0
$$

Details on how this boundary condition can be numerically enforced at a cloud center that belongs to the airfoil surface will be discussed in detail in section 5.2.

Instead of focusing on the specifics surrounding boundary condition enforcement, it will be assumed for the time being that there is an implicit way to make sure that both the farfield and wall boundary conditions are being correctly enforced and all of the flow variables at points located on these boundaries can be determined by solving the discretized form of the continuity equation. Therefore the remainder of this section will specifically focus on how the continuity equation is discretized.

Recall from Chapter 2 that the steady-state continuity equation in non-conservative form can be expressed in terms of the total velocity potential $\Phi$ :

$$
\rho\left(\frac{\partial^{2} \Phi}{\partial x^{2}}+\frac{\partial^{2} \Phi}{\partial y^{2}}\right)+\frac{\partial \rho}{\partial x} \frac{\partial \Phi}{\partial x}+\frac{\partial \rho}{\partial y} \frac{\partial \Phi}{\partial y}=0
$$

This equation can be discretized using the derivative coefficients determined from the previous section.

$$
\begin{gathered}
\rho_{0}^{n}\left[\sum_{i=1}^{n s} w_{i}^{2 x}\left(\Phi_{i}^{n}-\Phi_{0}^{n}\right)+\sum_{i=1}^{n s} w_{i}^{2 y}\left(\Phi_{i}^{n}-\Phi_{0}^{n}\right)\right]+ \\
\sum_{i=1}^{n s} w_{i}^{1 x}\left(\rho_{i}^{n}-\rho_{0}^{n}\right) \sum_{i=1}^{n s} w_{i}^{1 x}\left(\Phi_{i}^{n}-\Phi_{0}^{n}\right)+\sum_{i=1}^{n s} w_{i}^{1 y}\left(\rho_{i}^{n}-\rho_{0}^{n}\right) \sum_{i=1}^{n s} w_{i}^{1 y}\left(\Phi_{i}^{n}-\Phi_{0}^{n}\right)=0
\end{gathered}
$$

The goal is to re-arrange the terms in this algebraic equation to determine the updated $(n+1)$ value of the velocity potential. In order to put Equation (5.20) in a 
more compact form let:

$$
\begin{gathered}
\rho_{x}=\sum_{i=1}^{n s} w_{i}^{1 x}\left(\rho_{i}^{n}-\rho_{0}^{n}\right) \\
\rho_{y}=\sum_{i=1}^{n s} w_{i}^{1 y}\left(\rho_{i}^{n}-\rho_{0}^{n}\right) \\
\rho_{0}^{n} \sum_{i=1}^{n s}\left(w_{i}^{2 x}+w_{i}^{2 y}\right)\left(\Phi_{i}^{n}-\Phi_{0}^{n}\right)+ \\
\rho_{x} \sum_{i=1}^{n s} w_{i}^{1 x}\left(\Phi_{i}^{n}-\Phi_{0}^{n}\right)+\rho_{y} \sum_{i=1}^{n s} w_{i}^{1 y}\left(\Phi_{i}^{n}-\Phi_{0}^{n}\right)=0
\end{gathered}
$$

Equation (5.23) can then be split up into terms associated with the cloud center and terms associated with values at the cloud support points.

$$
\begin{gathered}
\sum_{i=1}^{n s}\left[\rho_{0}^{n}\left(w_{i}^{2 x}+w_{i}^{2 y}\right)+\rho_{x} w_{i}^{1 x}+\rho_{x} w_{i}^{1 y}\right] \Phi_{0}^{n}= \\
\sum_{i=1}^{n s}\left[\rho_{0}^{n}\left(w_{i}^{2 x}+w_{i}^{2 y}\right)+\rho_{x} w_{i}^{1 x}+\rho_{x} w_{i}^{1 y}\right] \Phi_{i}^{n}
\end{gathered}
$$

The updated value $\left(\Phi_{0}^{n+1}\right)$ can now be determined by solving Equation (5.24) for $\Phi_{0}$.

$$
\Phi_{0}^{n+1}=\frac{\sum_{i=1}^{n s}\left[\rho_{0}^{n}\left(w_{i}^{2 x}+w_{i}^{2 y}\right)+\rho_{x} w_{i}^{1 x}+\rho_{x} w_{i}^{1 y}\right] \Phi_{i}^{n}}{\sum_{i=1}^{n s}\left[\rho_{0}^{n}\left(w_{i}^{2 x}+w_{i}^{2 y}\right)+\rho_{x} w_{i}^{1 x}+\rho_{x} w_{i}^{1 y}\right]}
$$

Now that the value of the velocity potential has been updated, the velocity components and density can also be updated. As shown in previous chapters, the density is a function of the velocity field. The $x$ and $y$ components of the velocity are determined by numerically calculating the gradient of the velocity potential.

$$
U_{0}^{n+1}=\sum_{i=1}^{n s} w_{i}^{1 x}\left(\Phi_{i}^{n}-\Phi_{0}^{n+1}\right)
$$




$$
V_{0}^{n+1}=\sum_{i=1}^{n s} w_{i}^{1 y}\left(\Phi_{i}^{n}-\Phi_{0}^{n+1}\right)
$$

Once the velocity values are determined, the density ratio at the cloud center can be updated.

$$
\rho_{0}^{n+1}=\left[1+\frac{\gamma-1}{2} M_{\infty}^{2}\left(1-\left|\nabla \Phi_{0}^{n+1}\right|^{2}\right)\right]^{\frac{1}{\gamma-1}}
$$

\subsubsection{Convergence Estimation}

The convergence criteria for this algorithm will be defined as the maximum change

of the velocity potential between the current and the previous iteration step for all the points on the boundaries and in the domain. The solution will keep iterating until this quantity reaches a user specified tolerance value.

$$
\epsilon=\max \left(\left|\{\Phi\}^{n+1}-\{\Phi\}^{n}\right|\right)
$$

\subsection{Implementation Considerations}

The previous section outlined the basic steps that comprise the iterative algorithm developed to solve the full potential equation using the generalized finite difference method. This section will focus on specific issues associated with applying this algorithm to problems of engineering interest. These issues can be specifically categorized into boundary condition enforcement, correctly estimating derivatives near sharp corners, and accurately calculating gradients near the wake cut plane. This section will discuss in detail how each of these issues were addressed. 


\subsubsection{Neumann Boundary Condition Enforcement}

The primary method that has been used by several authors $[49,45,63]$ to enforce a Neumann boundary condition has been the use of ghost points. The procedure for generating these points and how they are used to implement the Neumann boundary condition will be discussed along with a new method that uses a Hermite type interpolation technique applied to the Taylor series least squares problem.

\section{Ghost Point Method}

The NASA report written by Batina [45] was one of the first to apply the finite point method to solve aerodynamic problems. Batina shows how the boundary conditions at an airfoil surface can be implicitly enforced by reflecting the domain points nearest to the wall along a local tangent line. The locations of the ghost points are determined by finding the minimal distance between the domain point and the airfoil surface and then reflecting the point along the local tangent vector of the surface. A typical ghost point configuration is depicted in Figure 5.4.

The value of the potential at these points can then be assigned to correspond to the correct boundary condition. The no normal flow boundary condition can be discretized by a central difference scheme of the following form.

$$
\left.\frac{\partial \Phi}{\partial \mathbf{n}}\right|_{B} \cong \frac{\Phi_{g}-\Phi_{d}}{2 \Delta n}=0
$$

The solution to Equation (5.30) shows that the value of the potential at the ghost node would need to be equal to the value of the potential at the associated domain 


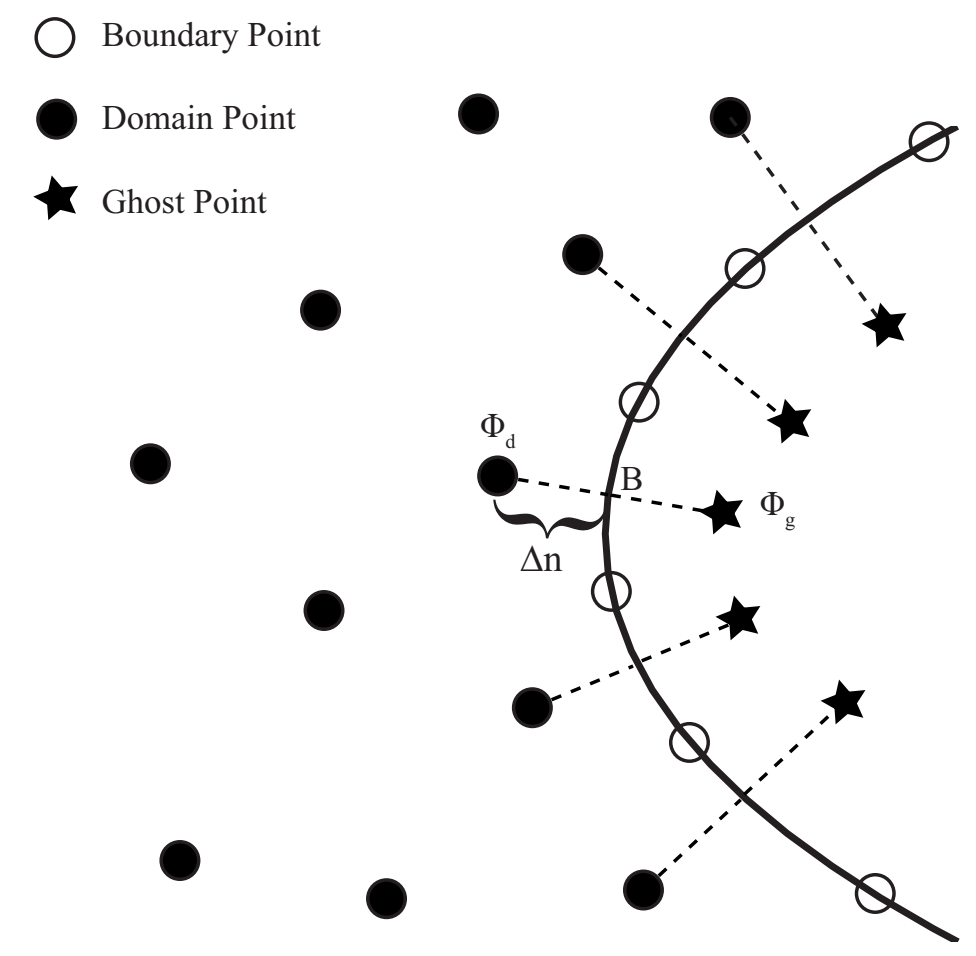

Figure 5.4: Ghost Points Near a Leading Edge

point. This method implicitly enforces the boundary condition and Katz [62] cites that the ghost nodes also "balance" the clouds associated with the boundary points which improves the conditioning of the matrices used to determine the derivative coefficients.

This method was initially implemented into the solution algorithm used in this thesis. Unfortunately the use of ghost points yielded inaccurate results. Tests were conducted to see how well the ghost point method can predict the velocity on the surface for the incompressible circular cylinder case. The converged solution underpredicted the magnitude of the surface velocities as shown in Figure 5.5. Due to 
this shortcoming, an investigation of another method that can accurately model the Neumann boundary condition was conducted.

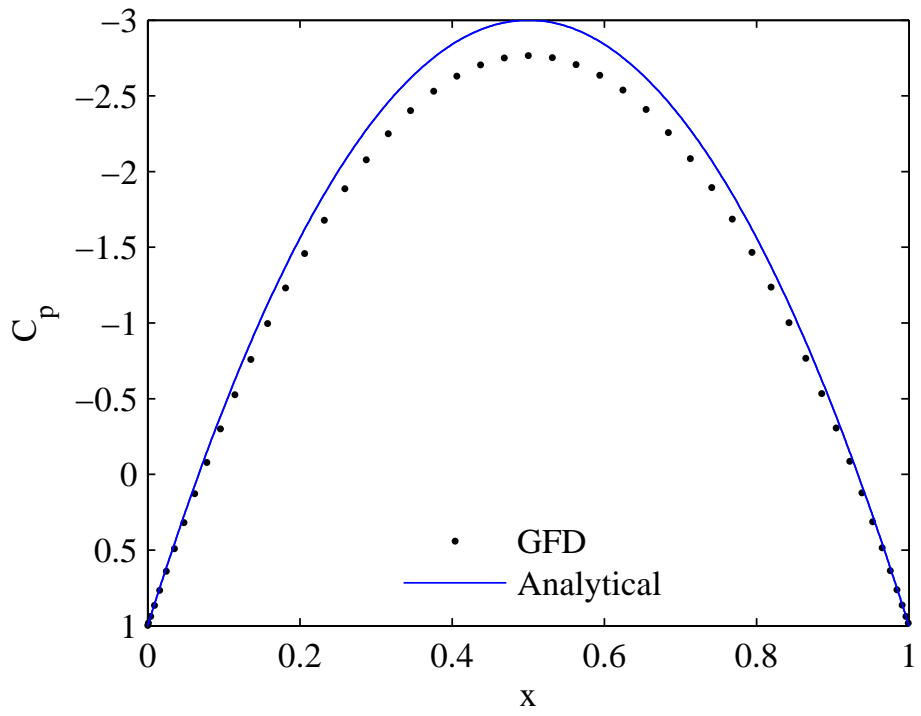

Figure 5.5: Cylinder $C_{p}$ Distribution Using Ghost Node Method

\section{Hermite Type Interpolation}

A new method was developed that uses the concept of Hermite interpolation.[64] Hermite interpolation involves approximating a function with a polynomial fit by using observations of both the function and its derivative.[65] This concept can be easily extended to a Taylor least squares fit on a cloud that includes Neumann boundary points. This section will outline how the Neumann boundary condition can be incorporated into the determination of the derivative coefficients that was explained in subsection 5.1.2.

When a cloud contains nodes that belong to a boundary with a specified Neumann boundary condition, a specialized form of the Taylor series least squares problem 
described in the previous section can be formulated. The new formulation takes advantage of the fact that both function values and function derivatives are known at the boundary nodes. Including this additional data results in a more accurate approximation of derivatives at the cloud center.

If there is a subset of $n b$ boundary nodes within the $n s$ supporting nodes of the cloud centered at $\left(x_{0}, y_{0}\right)$, the truncated Taylor series for the first $x$ and $y$ derivatives at $\left(x_{j}, y_{j}\right),[j \in 1 \ldots n b]$ can be expressed as

$$
\begin{gathered}
\frac{\partial \Phi_{j}}{\partial x}=\sum_{q=1}^{l} \sum_{m=0}^{q}\left(\begin{array}{c}
q \\
m
\end{array}\right)(q-m) \frac{\Delta x_{j}^{q-m-1} \Delta y_{j}^{m}}{q !} \frac{\partial^{q} \Phi_{0}}{\partial x^{q-m} \partial y^{m}} \\
\frac{\partial \Phi_{j}}{\partial y}=\sum_{q=1}^{l} \sum_{m=0}^{q}\left(\begin{array}{c}
q \\
m
\end{array}\right)(m) \frac{\Delta x_{j}^{q-m} \Delta y_{j}^{m-1}}{q !} \frac{\partial^{q} \Phi_{0}}{\partial x^{q-m} \partial y^{m}}
\end{gathered}
$$

The gradient of the potential in the normal direction $\nabla \phi_{j} \cdot \mathbf{n}_{j}$ can be expressed in terms of the Taylor series by taking the dot product of a vector consisting of Equations

$$
\begin{aligned}
& \text { and (5.32) }\left(\left[\frac{\partial \Phi_{j}}{\partial x}, \frac{\partial \Phi_{j}}{\partial y}\right]\right) \text { with the normal vector } \mathbf{n}_{j}\left(\left[n_{x_{j}}, n_{y_{j}}\right]\right) \\
& \qquad \nabla \Phi_{j} \cdot \mathbf{n}_{j}= \\
& \sum_{q=1}^{l} \sum_{m=0}^{q}\left(\begin{array}{c}
q \\
m
\end{array}\right) \frac{1}{q !}(q-m) n_{x_{j}} \Delta x_{j}^{q-m-1} \Delta y_{j}^{m} \frac{\partial^{q} \Phi_{0}}{\partial x^{q-m}+\partial y^{m}}+ \\
& \sum_{q=1}^{l} \sum_{m=0}^{q}\left(\begin{array}{c}
q \\
m
\end{array}\right) \frac{1}{q !}(m) n_{y_{j}} \Delta x_{j}^{q-m} \Delta y_{j}^{m-1} \frac{\partial^{q} \Phi_{0}}{\partial x^{q-m} \partial y^{m}}
\end{aligned}
$$

The derivatives of the function can be found by applying a least squares minimization problem. A weighted residual statement can now be made on the Taylor series for the $n b$ nodes where $w_{j}$ is the same inverse distance weighting function from 
Equation (5.6).

$$
\begin{gathered}
R_{2}=\sum_{j=1}^{n b} w_{j}\left[\left(\nabla \Phi_{j} \cdot \mathbf{n}_{j}\right)-\left(\sum_{q=1}^{l} \sum_{m=0}^{q}\left(\begin{array}{c}
q \\
m
\end{array}\right) \frac{1}{q !}(q-m) n_{x_{j}} \Delta x_{j}^{q-m-1} \Delta y_{j}^{m} \frac{\partial^{q} \Phi_{0}}{\partial x^{q-m} \partial y^{m}}+\right.\right. \\
\left.\left.\left(\begin{array}{c}
q \\
m
\end{array}\right) \frac{1}{q !}(m) n_{y_{j}} \Delta x_{j}^{q-m} \Delta y_{j}^{m-1} \frac{\partial^{q} \Phi_{0}}{\partial x^{q-m} \partial y^{m}}\right)\right]^{2}
\end{gathered}
$$

Minimizing this residual with respect to each of the $l\left(\frac{l+3}{2}\right)$ derivatives results in a system of equations of the form

$$
[C][D]\{d\}=[C]\{n\}
$$

The $q$ and $m$ coefficients can be determined in a systematic manner by applying the same algorithm from the previous section. The elements in the matrix $[C]$ follow the same pattern as the matrix $[A]$ from the previous section.

$$
\begin{aligned}
& c_{1,1}= w_{1}\left[\left(\begin{array}{c}
q_{1} \\
m_{1}
\end{array}\right) \frac{1}{q_{1} !}\left(q_{1}-m_{1}\right) n_{x_{1}} \Delta x_{1}^{q_{1}-m_{1}-1} \Delta y_{1}^{m_{1}}\right]+ \\
& w_{1}\left[\left(\begin{array}{c}
q_{1} \\
m_{1}
\end{array}\right) \frac{1}{q_{1} !}\left(m_{1}\right) n_{y_{1}} \Delta x_{1}^{q_{1}-m_{1}} \Delta y_{1}^{m_{1}-1}\right] \\
& c_{1, n b}=w_{n b}\left[\left(\begin{array}{c}
q_{1} \\
m_{1}
\end{array}\right) \frac{1}{q_{1} !}\left(q_{1}-m_{1}\right) n_{x_{n b}} \Delta x_{n b}^{q_{1}-m_{1}-1} \Delta y_{n b}^{m_{1}}\right]+ \\
& w_{n b}\left[\left(\begin{array}{c}
q_{1} \\
m_{1}
\end{array}\right) \frac{1}{q_{1} !}\left(m_{1}\right) n_{y_{n b}} \Delta x_{n b}^{q_{1}-m_{1}} \Delta y_{n b}^{m_{k}-1}\right] \\
& c_{t, 1}=w_{1}\left[\left(\begin{array}{c}
q_{t} \\
m_{t}
\end{array}\right) \frac{1}{q_{t} !}\left(q_{t}-m_{t}\right) n_{x_{1}} \Delta x_{1}^{q_{t}-m_{t}-1} \Delta y_{1}^{m_{t}}\right]+
\end{aligned}
$$




$$
\begin{aligned}
& w_{1}\left[\left(\begin{array}{c}
q_{t} \\
m_{t}
\end{array}\right) \frac{1}{q_{t} !}\left(m_{t}\right) n_{y_{1}} \Delta x_{1}^{q_{t}-m_{t}} \Delta y_{1}^{m_{t}-1}\right] \\
& c_{t, n b}=w_{n b}\left[\left(\begin{array}{c}
q_{t} \\
m_{t}
\end{array}\right) \frac{1}{q_{t} !}\left(q_{t}-m_{t}\right) n_{x_{n b}} \Delta x_{n b}^{q_{t}-m_{t}-1} \Delta y_{n b}^{m_{t}}\right]+ \\
& w_{n b}\left[\left(\begin{array}{c}
q_{t} \\
m_{t}
\end{array}\right) \frac{1}{q_{t} !}\left(m_{t}\right) n_{y_{n b}} \Delta x_{n b}^{q_{t}-m_{t}} \Delta y_{n b}^{m_{t}-1}\right] \\
& {[C]=\left[\begin{array}{ccc}
c_{1,1} & \cdots & c_{1, n b} \\
\vdots & \ddots & \vdots \\
c_{t, 1} & \cdots & c_{t, n b}
\end{array}\right]}
\end{aligned}
$$

The $[D]$ matrix is the transpose of $[C]$ with the inverse distance weighting function excluded.

$$
\begin{aligned}
d_{1,1}= & {\left[\left(\begin{array}{c}
q_{1} \\
m_{1}
\end{array}\right) \frac{1}{q_{1} !}\left(q_{1}-m_{1}\right) n_{x_{1}} \Delta x_{1}^{q_{1}-m_{1}-1} \Delta y_{1}^{m_{1}}\right]+} \\
& {\left[\left(\begin{array}{c}
q_{1} \\
m_{1}
\end{array}\right) \frac{1}{q_{1} !}\left(m_{1}\right) n_{y_{1}} \Delta x_{1}^{q_{1}-m_{1}} \Delta y_{1}^{m_{1}-1}\right] } \\
d_{1, t}= & {\left[\left(\begin{array}{c}
q_{t} \\
m_{t}
\end{array}\right) \frac{1}{q_{t} !}\left(q_{t}-m_{t}\right) n_{x_{1}} \Delta x_{1}^{q_{t}-m_{t}-1} \Delta y_{1}^{m_{t}}\right]+} \\
& {\left[\left(\begin{array}{c}
q_{t} \\
m_{t}
\end{array}\right) \frac{1}{q_{1} !}\left(m_{t}\right) n_{y_{t}} \Delta x_{1}^{q_{t}-m_{t}} \Delta y_{1}^{m_{t}-1}\right] } \\
d_{n b, 1}= & {\left[\left(\begin{array}{c}
q_{1} \\
m_{1}
\end{array}\right) \frac{1}{q_{1} !}\left(q_{1}-m_{1}\right) n_{x_{n b}} \Delta x_{n b}^{q_{1}-m_{1}-1} \Delta y_{n b}^{m_{1}}\right]+} \\
& {\left[\left(\begin{array}{c}
q_{1} \\
m_{1}
\end{array}\right) \frac{1}{q_{1} !}\left(m_{1}\right) n_{y_{n b}} \Delta x_{n b}^{q_{1}-m_{1}} \Delta y_{n b}^{m_{k}-1}\right] } \\
d_{n b, t}= & {\left[\left(\begin{array}{c}
q_{t} \\
m_{t}
\end{array}\right) \frac{1}{q_{t} !}\left(q_{t}-m_{t}\right) n_{x_{n b}} \Delta x_{n b}^{q_{t}-m_{t}-1} \Delta y_{n b}^{m_{t}}\right]+}
\end{aligned}
$$




$$
\begin{gathered}
{\left[\left(\begin{array}{c}
q_{t} \\
m_{t}
\end{array}\right) \frac{1}{q_{t} !}\left(m_{t}\right) n_{y_{n b}} \Delta x_{n b}^{q_{t}-m_{t}} \Delta y_{n b}^{m_{t}-1}\right]} \\
{[D]=\left[\begin{array}{ccc}
d_{1,1} & \cdots & d_{1, t} \\
\vdots & \ddots & \vdots \\
d_{n b, 1} & \cdots & d_{n b, t}
\end{array}\right]}
\end{gathered}
$$

The vector $\{d\}$ contains the same elements as it did in the previous section. The vector $\{n\}$ is defined as the values of the Neumann boundary condition at each of the $n b$ nodes.

$$
\{n\}=\left[\begin{array}{c}
\nabla \Phi_{1} \cdot \mathbf{n}_{1} \\
\vdots \\
\nabla \Phi_{n b} \cdot \mathbf{n}_{n b}
\end{array}\right]
$$

Now that the method for solving the least square problem associated with derivative data at the boundary nodes has been established, it can be added to the least squares problem associated with the function data at all the nodes in the cloud by adding Equation (5.8) to Equation (5.35)

$$
[[A][B]+[C][D]]\{d\}=[A]\{b\}+[C]\{n\}
$$

If the boundary is an impermeable wall, the Neumann boundary condition becomes:

$$
\nabla \Phi_{j} \cdot \mathbf{n}_{j}=0
$$

which implies that the second term on the right hand side of Equation (5.47) is a vector of zeros.

$$
[[A][B]+[C][D]]\{d\}=[A]\{b\}
$$

The $[T]$ matrix from the previous section is now defined as

$$
[T]=[[A][B]+[C][D]]^{-1}[A]
$$


and the derivative coefficients are also expressed the same way as in the previous section.

\subsubsection{Trailing Edge Treatment}

Special restrictions on the choice of cloud points is applied when a cloud center is in the vicinity of an airfoil trailing edge. The area within the airfoil surface is considered to be outside the domain. Therefore a cloud cannot contain nodes that cross over the surface of the airfoil. This situation is illustrated below for a point near the trailing edge. A similar problem is encountered in crack propagation problems in structural mechanics. Nguyen et. al. [43] described a node selection criteria known as the visibility method when considering cloud points near a discontinuous surface such as a crack. The discontinuous surface is treated as opaque. A ray is then calculated between the cloud center and the candidate support node. If the ray intersects the discontinuous surface, the node is not selected as a support point since it is "invisible" to the cloud center. The same conditions are enforced at the trailing edge since it can be considered as a discontinuous surface because there is no continuous change in the potential across the airfoil surface.

If the invisible nodes were selected as support points, the flow solution would be inaccurate near the trailing edge and in some cases cause a divergent solution. Figure 5.7 illustrates this effect for the prediction of pressure coefficient on the surface for a converged solution of a NACA 0012 airfoil in incompressible flow. The inaccurate results at the trailing edge are spurious and not smooth due to the error introduced 


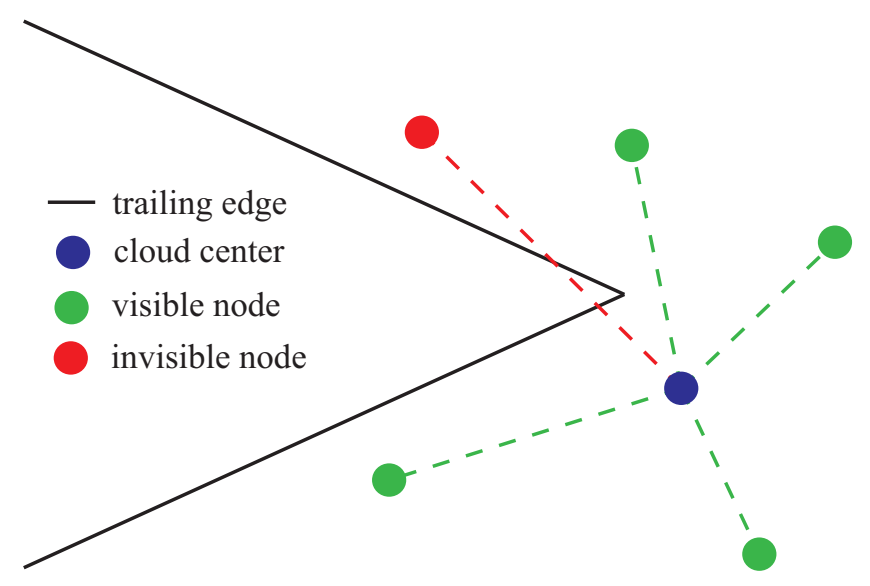

Figure 5.6: Visibility Method

from including points crossing the trailing edge surface. These errors do not extend across the entire surface because the nearest neighbors for points on a thicker portion of the airfoil remain on the visible side.

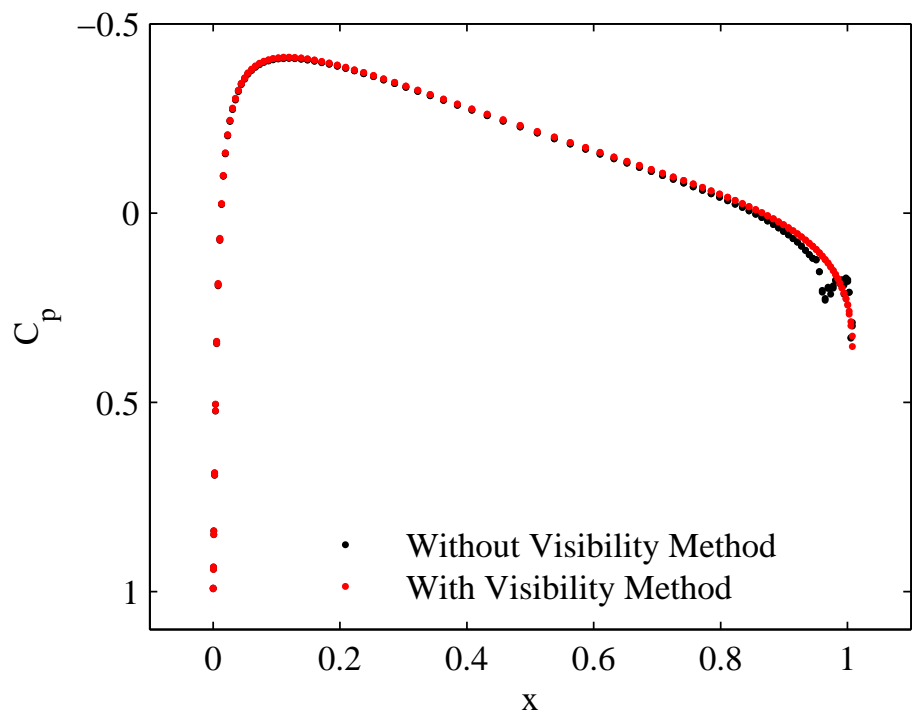

Figure 5.7: Bad Trailing Edge Results 


\subsubsection{Wake Treatment}

The Kutta-Joukowski theorem states that circulation is non-zero in lifting flows.[3] Circulation can be represented in two-dimensional flows by a constant strength doublet distribution emanating from the trailing edge to the downstream farfield boundary or by an equivalent point vortex located at the trailing edge of the airfoil. Both representations result in an instantaneous jump condition in velocity potential across the wake cut plane. This scenario is depicted in Figure 5.8 for the perturbation velocity field of a NACA 0012 airfoil at a $2^{\circ}$ angle of attack. When a cloud center is near the wake cut plane special considerations need to be made in order for the flow variables to be correctly predicted.

While Figure 5.8 clearly shows that the velocity potential is discontinuous across the wake cut plane, $\nabla \Phi$ is continuous through the wake.[66] If two points are sufficiently close to the wake but are located on opposite sides, it is assumed there is a linear change in potential across the wake cut plane. By making these assumptions, a simple correction to the values of the potential when clouds contain support points that are on both sides of the wake cut plane can be made in order to correctly calculate the velocity. Figure 5.9 is taken from Reference [66] and illustrates how the value of the potential is adjusted depending on which side of the wake cut plane the support node is located.

If the cloud center is located above the wake cut plane and it has a support point

located at point $\mathrm{A}$, the adjusted potential value $\Phi_{A^{*}}$ is equal to the original value of 


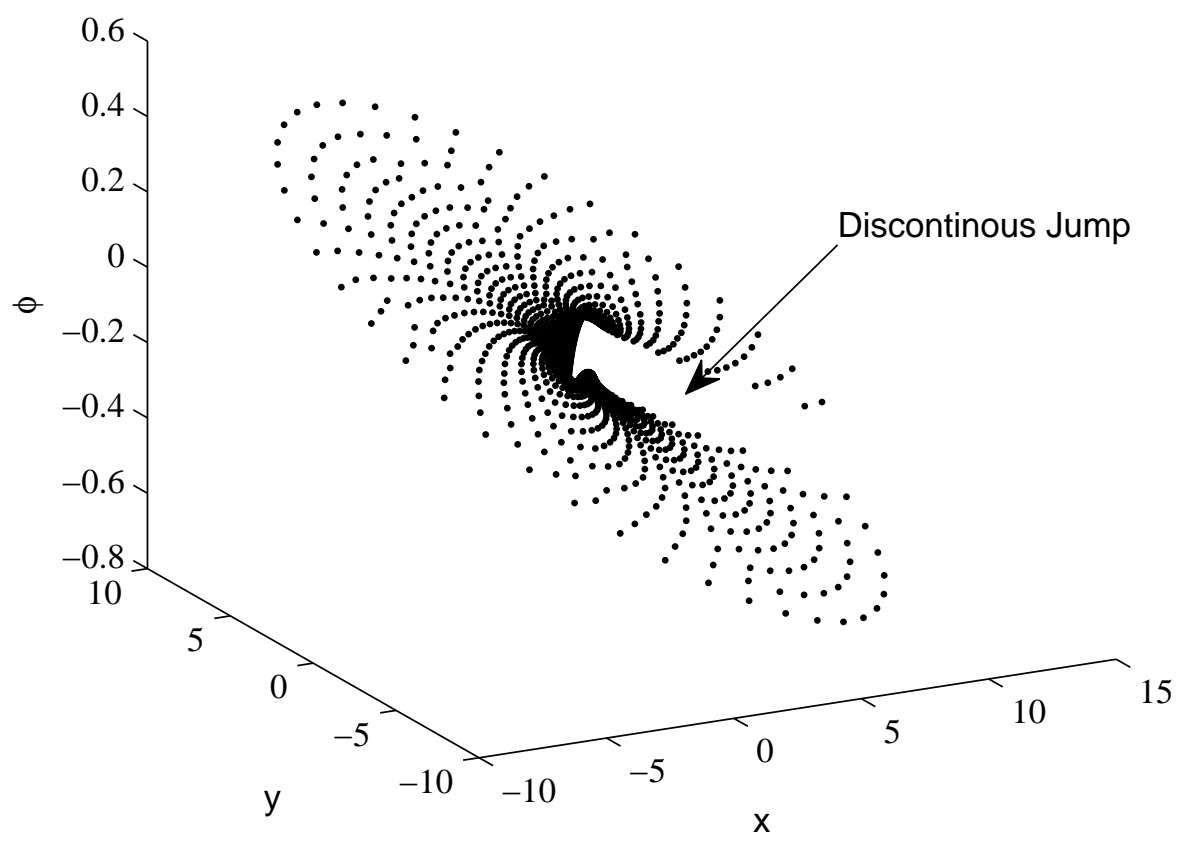

Figure 5.8: Discontinous Potential Field

the potential $\Phi_{A}$ in addition to the circulation value $\Gamma$.

$$
\Phi_{A^{*}}=\Phi_{A}+\Gamma
$$

The opposite is true when the cloud center is located below the wake cut plane and a support point is above the wake. In this situation the adjusted value of the velocity potential $\left(\Phi_{B^{*}}\right)$ at point $\mathrm{B}$ is equal to the circulation subtracted from the original value $\Phi_{B}$.

$$
\Phi_{B^{*}}=\Phi_{B}-\Gamma
$$




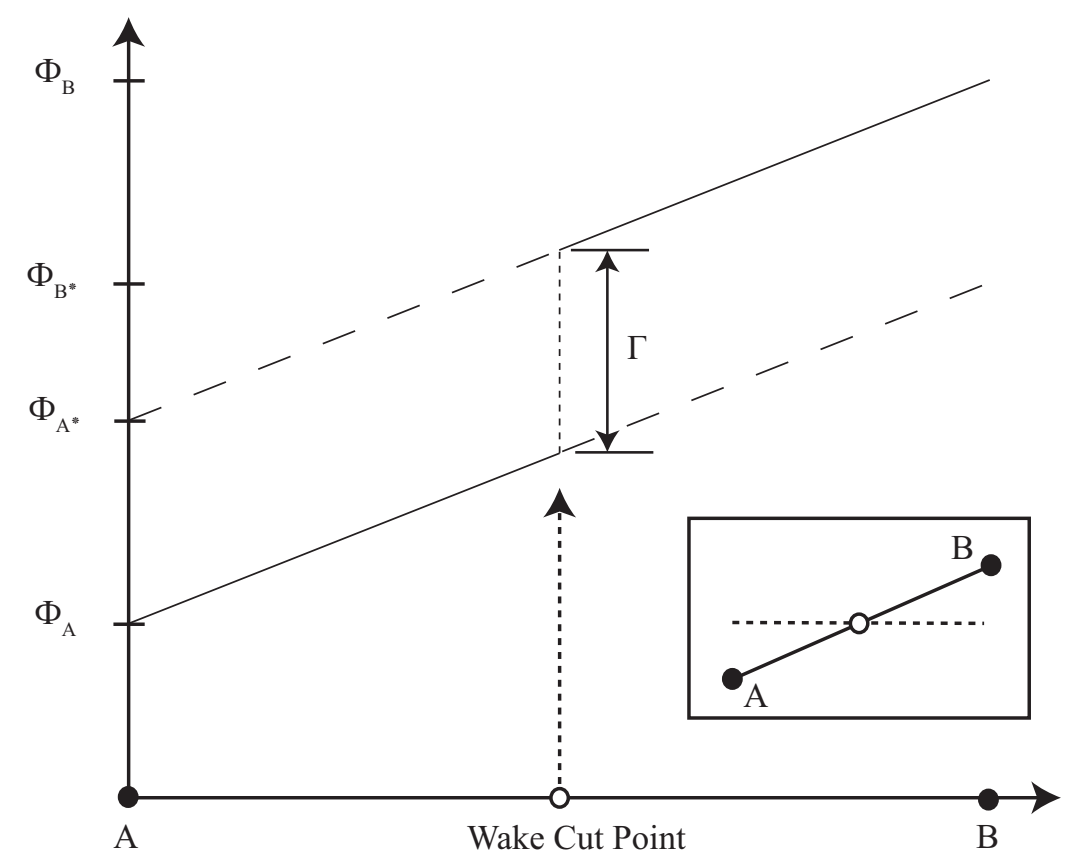

Figure 5.9: Variation of $\Phi$ Across Wake

\subsection{Order of Accuracy Estimates}

One of the verification studies that needs to be performed for standard mesh based flow solvers is to assess the order of accuracy of the scheme employed to estimate the partial derivatives. Both the ASME Journal of Fluids Engineering [67] and AIAA Journal of Aircraft [68] require that a published solution must use at least a second order accurate scheme in space. This section will be devoted to showing that the generalized finite difference method can meet or exceed this requirement.

The first section of this chapter showed that the generalized finite difference method uses a truncated Taylor series to determine the derivative coefficients. This Taylor series can keep an arbitrary number of terms with a higher number of terms 
corresponding to a higher order estimation. For example Equation (5.4) with $l=2$ corresponds to the Taylor series that retains the second order terms.

$$
\begin{gathered}
\Delta \Phi_{i}=\Delta x_{i} \frac{\partial \Phi_{0}}{\partial x}+\Delta y_{i} \frac{\partial \Phi_{0}}{\partial x}+ \\
\frac{\Delta x_{i}^{2}}{2} \frac{\partial^{2} \Phi_{0}}{\partial x^{2}}+\Delta x_{i} \Delta y_{i} \frac{\partial^{2} \Phi_{0}}{\partial x \partial y}+\frac{\Delta y_{i}^{2}}{2} \frac{\partial^{2} \Phi_{0}}{\partial y^{2}}+O\left(\Delta x_{i}^{3}, \Delta y_{i}^{3}\right)
\end{gathered}
$$

Sridar and Balakrishnan [57] proved theoretically that the order of the leading truncation error term for the estimate of the $p_{t h}$ derivative of a function is $h^{l+1-p}$ where $h$ is the characteristic length in the Taylor series expansion and $l$ is the highest order of the terms kept in the Taylor expansion.

The presentation provided by Sridar and Balakrishnan was for one dimension but they stated that it could easily be extended to higher dimensions. The question remains what the characteristic distance would be for a multidimensional problem. The mean point spacing within the cloud as suggested by Morinishi [59] will be used as the distance to measure the order of accuracy for a two dimensional calculation.

$$
d_{m}=\frac{1}{n s} \sum_{i=1}^{n s} \sqrt{\left(x_{i}-x_{0}\right)^{2}+\left(y_{i}-y_{0}\right)^{2}}
$$

Applying the theoretical order of accuracy criterion established by Sridar and Balakrishnan suggests that the discretization error associated with the gradient of the velocity potential using the Taylor series in Equation (5.53) would be on the order of $d_{m}{ }^{2}$ and the discretization error associated with the Laplacian would be on the order of $d_{m}$. 
The next two subsections will reproduce a study similar to the one conducted by Morinishi [59] which numerically determined that the order of accuracy for clouds using a polynomial based least squares technique.

\subsubsection{In Domain Discretization Error}

The analytical solution for the incompressible flow around a circular cylinder will be used as the exact solution for both the velocities and accelerations used in this verification exercise. The velocities were presented in polar coordinates in Chapter 3. The velocities and accelerations will be reproduced here in Cartesian coordinates.

$$
\begin{gathered}
U=U_{\infty}\left(1-\frac{R^{2}}{r^{2}}\right)+\frac{2 R^{2} y^{2}}{r^{4}} \\
V=\frac{-2 U_{\infty} x y R^{2}}{r^{4}} \\
\frac{\partial U}{\partial x}=U_{\infty} x\left(\frac{2 R^{2}}{r^{4}}-\frac{8 R^{2} y^{2}}{r^{6}}\right) \\
\frac{\partial V}{\partial y}=-U_{\infty} x\left(\frac{2 R^{2}}{r^{4}}-\frac{8 R^{2} y^{2}}{r^{6}}\right)
\end{gathered}
$$

An unstructured mesh was created around a circular cylinder centered at $(0.5,0.0)$ with a radius of 0.5 using the mesh generator GAMBIT. The derivatives at an off-body point located at $\left(-6.399 \times 10^{-4}, 0.563\right)$ were calculated and compared to the analytical solution. These coordinates correspond to an off-body point that is sufficiently close to the cylinder surface yet all of its support points are not located on the boundary.

Figure 5.10 shows the cloud containing these nine nearest neighbors to the test point. The amount of support points was chosen to be nine because it is the minimum 
amount required for a third order Taylor series fit. According to the Sridar and Balakrishnan's criterion, it is expected that a third order $(l=3)$ fit will be able to discretize the second $(p=2)$ derivative of the velocity potential with second order accuracy.

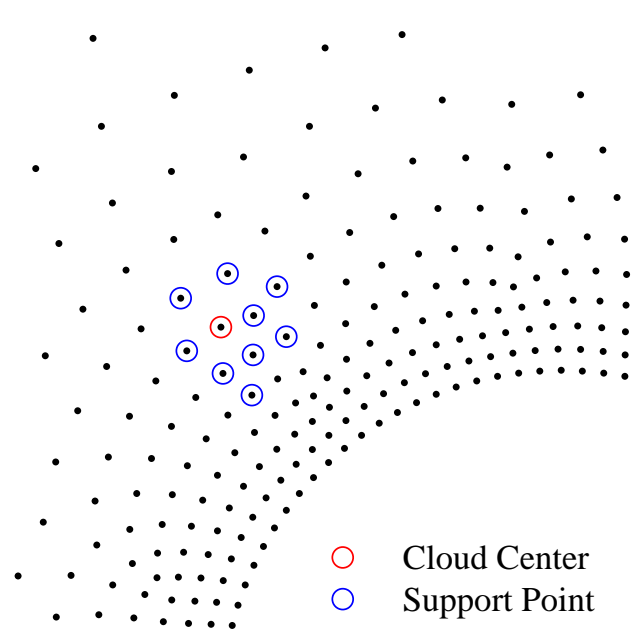

Figure 5.10: Domain Cloud

The coarsest level is the cloud configuration depicted in Figure 5.10. Subsequent refinements are performed by scaling the cloud support points by a factor k:

$$
\begin{gathered}
x_{i}^{f}=x_{0}+k\left(x_{i}^{c}-x_{0}\right) \\
y_{i}^{f}=y_{0}+k\left(y_{i}^{c}-y_{0}\right)
\end{gathered}
$$

where $f$ and $c$ correspond to the fine and coarse coordinates. The cloud support points were scaled from $k=1$ to $k=10^{-6}$. Figure 5.11 shows the errors associated with predicting the flowfield velocities and accelerations. As expected the discretization 
error associated with calculating the $x$ and $y$ components of the velocity is on the order of $d_{m}{ }^{3}$ and the error associated with the second derivatives of the velocity potential is on the order of $d_{m}{ }^{2}$. This numerical study confirms that for clouds containing only domain points the Taylor series fit with $l=3$ is at least second order accurate for all derivatives that need to be calculated in the solution procedure.
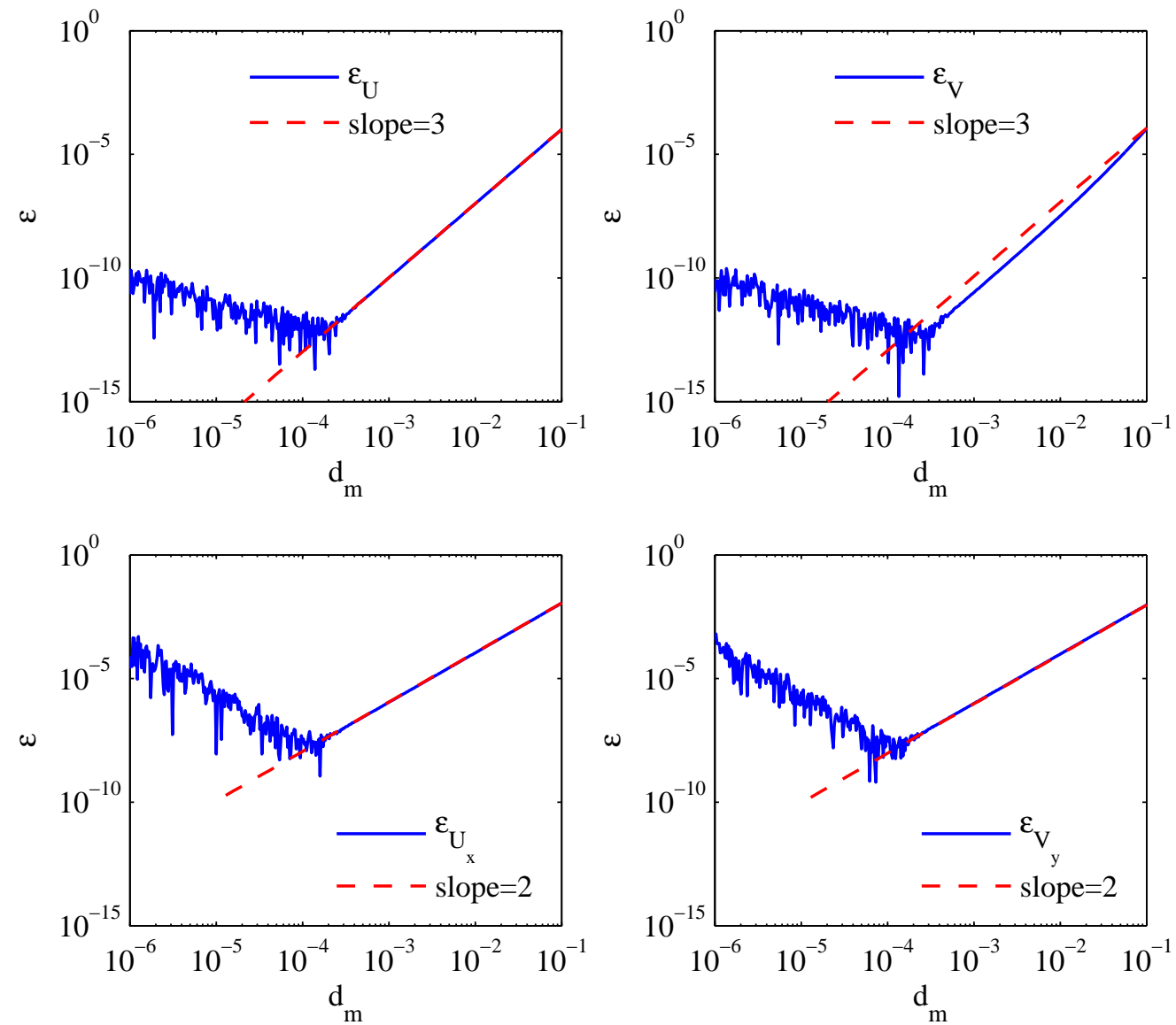

Figure 5.11: Domain Point Discretization Error in Estimation of 1st and 2nd Derivatives 


\subsubsection{On Boundary Discretization Error}

A numerical study was also conducted to determine the order of accuracy for clouds containing Neumann boundary points in order to confirm that the Hermite type enforcement of the boundary condition retains the same discretization error properties of a domain only point cloud. As a test case the cloud center was selected to be located at the upper suction peak of the circle $(0.5,0.5)$. Once again the nine nearest neighbors were selected as the support points which corresponds to four support points located on the boundary and five support points located in the domain as depicted in Figure 5.12.

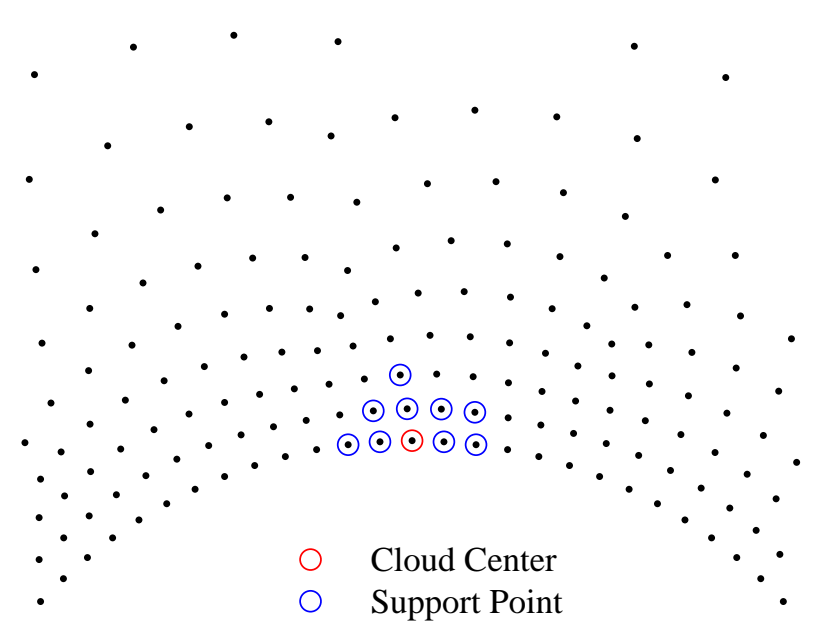

Figure 5.12: Boundary Cloud

The $x$ coordinates of the boundary support points were scaled in the same manner as the domain points but the $y$ coordinates were then calculated by substituting the $x$ coordinates into the equation for the upper surface of a circle. This would ensure 
that the boundary points remain on the cylinder surface for every refinement level.

$$
y_{i}^{f}=\sqrt{0.5^{2}-\left(x_{i}^{f}-0.5\right)^{2}}
$$

The errors associated with both the first and second derivatives of the velocity potential closely mirror the same behavior exhibited by the domain only point cloud. Figure 5.11 shows that the discretization error associated with the $x$ and $y$ components of the velocity along with the acceleration of the flow in the $\mathrm{x}$ direction exhibit the behavior predicted by Sridar and Balakrishnan. An anomaly associated with the error in calculating $\frac{\partial V}{\partial y}$ occurs. The error drops off quite drastically around $d_{m}=10^{-2}$ and then rapidly comes back to what the expected value would be if the error was dropping off in a linear manner. While this behavior is unexpected, the discretization error never drops off on an order above $d_{m}{ }^{2}$. 

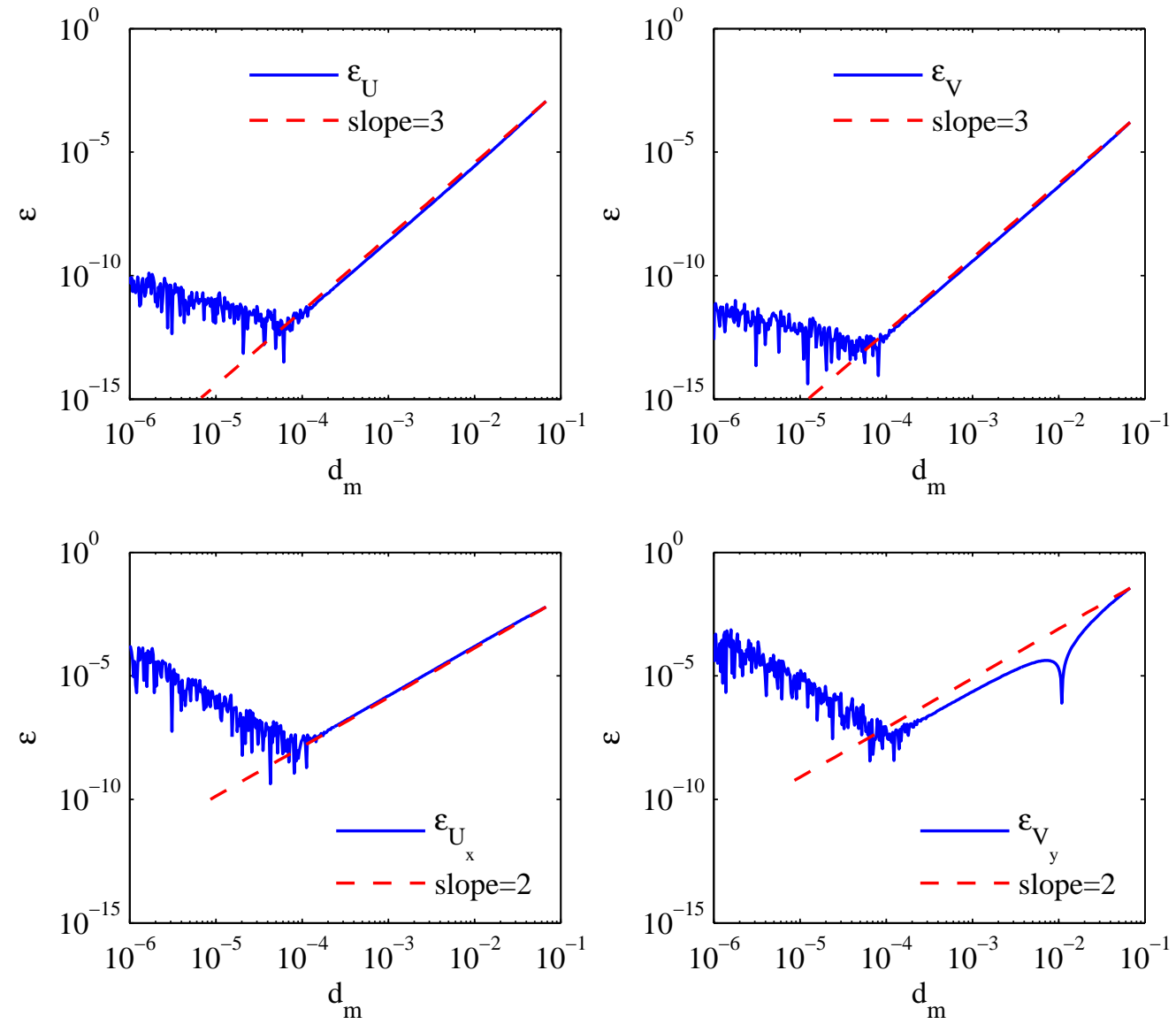

Figure 5.13: Boundary Point Discretization Error 


\section{Chapter 6}

\section{Genralized Finite Difference Method Results}

The generalized finite difference method was applied to solve a variety of nonlifting and lifting subcritical compressible cases. The first two cases are identical to the ones used in the previous chapter. That way it can be determined how well the GFD method can perform compared to the dual reciprocity method. In order to examine how well the method can solve lifting flows, the flow over a NACA 0012 at a two degree angle of attack was solved. For each of these cases a "grid" convergence study was conducted to first show that the meshless technique can be verified in a similar manner to common CFD algorithms and also to explore what kind of point distribution is adequate for accurately solving the full potential equation.

\subsection{Circular Cylinder}

The first case that was examined was the sub-critical flow over a circular cylinder. As with the case solved by the dual reciprocity method, the freestream Mach number 
is 0.375 and the suction peak velocity for several node distributions was compared to the analytical solution $\left(V_{\max }=2.260\right)$.[26] All of the node distributions were generated by the unstructured mesh generator GAMBIT. The baseline node distribution, which has 100 nodes uniformly distributed on the cylinder surface, is shown in Figures 6.1 and 6.2. The farfield boundary was chosen to be a circle with radius 20 measured from the circular cylinder center. The domain nodes were grown off the surface distribution to the farfield boundary using a 1.2 growth ratio. The influence of these metrics, which are summarized in Table 6.1, on the accuracy of the predicted peak velocity were examined along with the effects of the number of cloud support points in order to systematically test the convergence and accuracy properties of the generalized finite difference method.

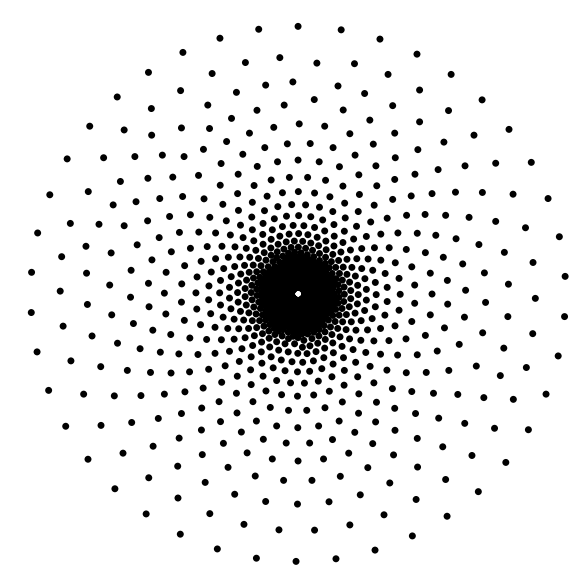

Figure 6.1: Baseline Circular Cylinder Node Distribution 


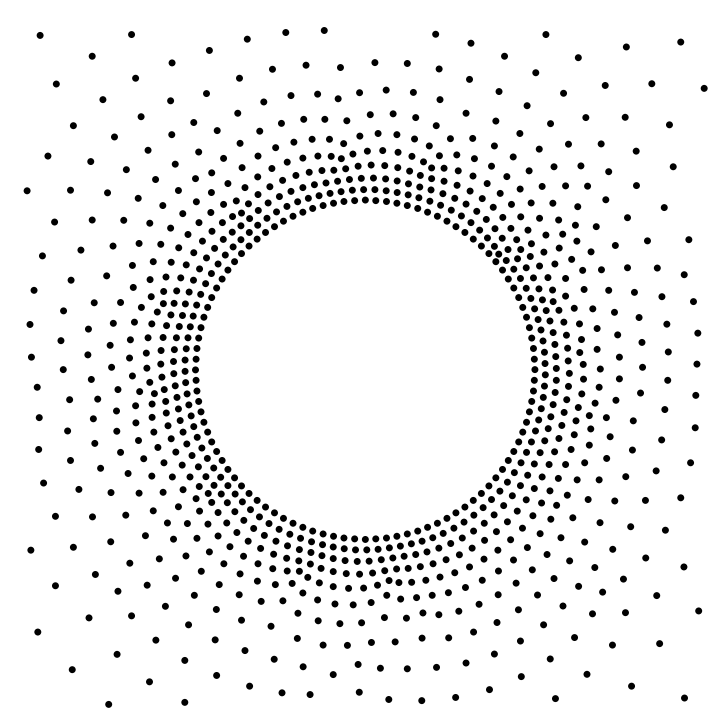

Figure 6.2: Close-Up View of Baseline Circular Cylinder Node Distribution

Table 6.1: Baseline Distribution Metrics

\begin{tabular}{cc}
\hline \hline Number of Boundary Nodes & 100 \\
Farfield Boundary Radius & 20 \\
Cell Growth Ratio & 1.2 \\
Total Number of Nodes & 1971 \\
Number of Support Points & 18 \\
\hline
\end{tabular}

The results from the converged solution on the baseline node distribution are presented in the next three figures. Figure 6.3 shows that the convergence of the solution is quite smooth and predictable. The predicted pressure coefficient distribution, as shown in Figure 6.4, matches very closely with the results obtained by German [27] and Van Dyke [26]. Unlike the Dual Reciprocity Method, no a-priori knowledge of the solution was needed to tune any parameters used to solve the PDE. Figure 6.5 shows the value of $\frac{\partial \Phi}{\partial \mathbf{n}}$ on the surface of the cylinder in order to assess how well the 
Hermite type interpolation enforced the no normal flow boundary condition. These values are on the order of $10^{-3}$ which indicates that the boundary condition is being enforced with an adequate level of accuracy.

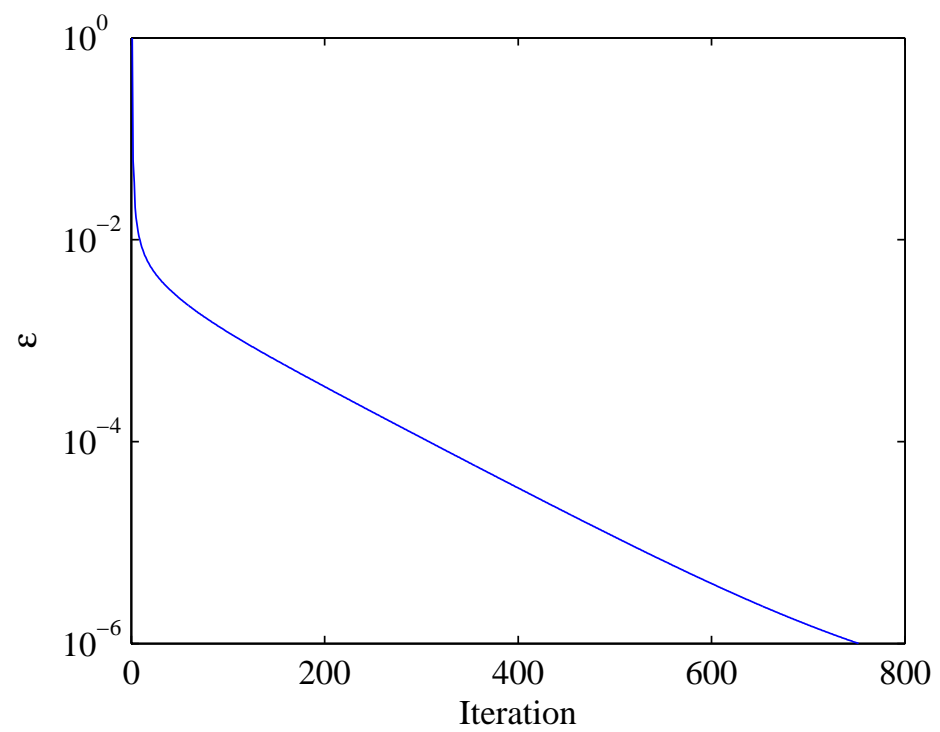

Figure 6.3: Convergence History for Baseline Node Distribution

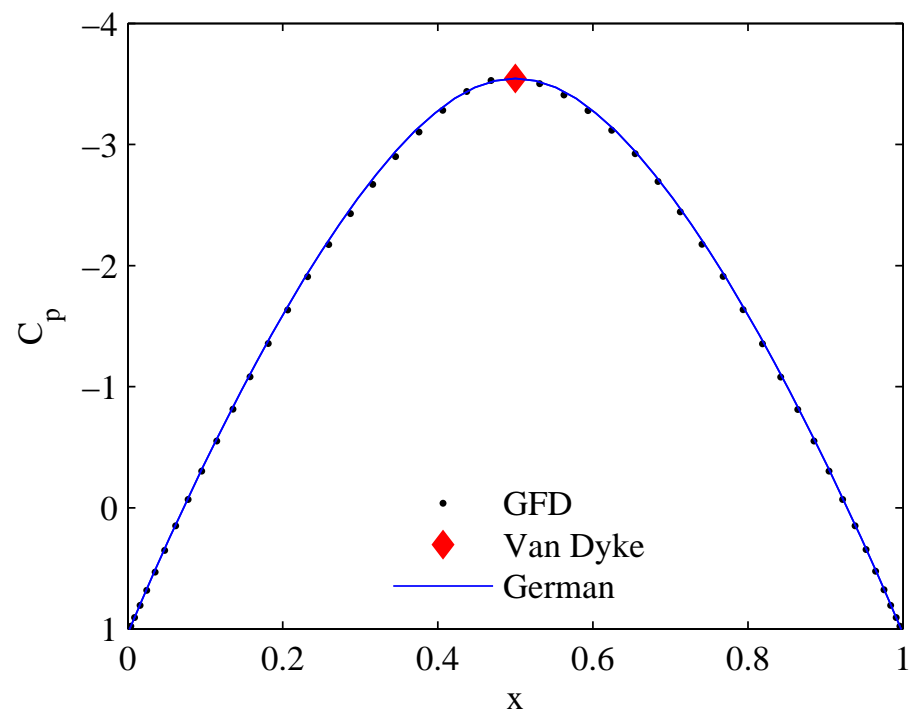

Figure 6.4: Upper Surface Pressure Coefficient for the Baseline Node Distribution 


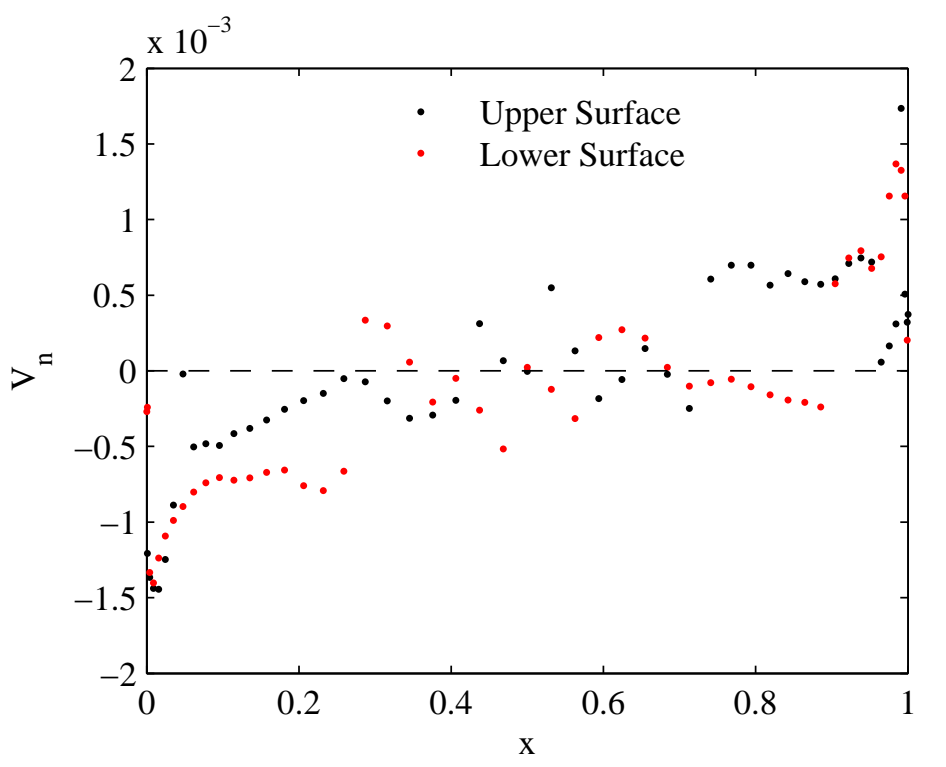

Figure 6.5: Normal Velocity Component on Cylinder Surface

\subsubsection{Convergence Criteria}

Before the node distribution study could be conducted, it was important to determine when the iterative solution had converged to an asymptotic final answer. Figure 6.6 shows how the error between the calculated peak velocity and the analytical solution changes as a function of the iteration error defined in the convergence criteria section of the previous chapter. It is apparent that the velocity shows minimal change once the iteration error reaches a value of approximately $10^{-5}$. Therefore the solution will be considered converged when the iteration error reaches a conservative value of $10^{-6}$ for all subsequent studies. 


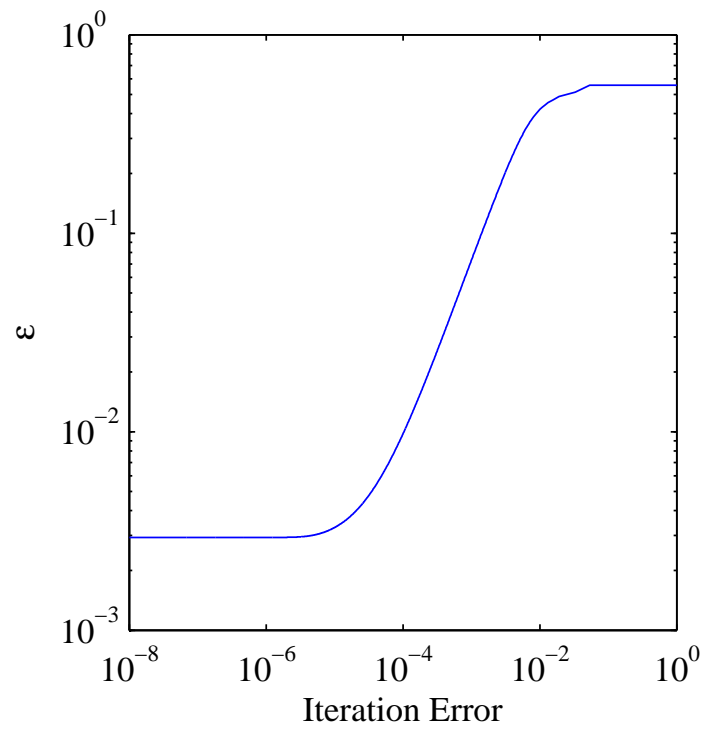

Figure 6.6: Evolution of Suction Peak Velocity Error

\subsubsection{Number of Support Points}

The next study that was performed examined how the number of support points in the local cloud influences the prediction of the suction peak velocity. Since the truncated series used in the determination of the derivative coefficients includes partial derivatives up to the third order $(l=3)$, the minimum amount of support points required for all of the clouds is $l\left(\frac{l+3}{2}\right)=9$. This amount of support points was chosen initially, but a convergent solution could not be obtained. This unstable convergence property may be caused by an inadequate density of nodes, but the exact cause has not been found. It was determined that the minimum amount of support points that could adequately solve the baseline node distribution was 12 . To study the effects of increasing the number of support points from this minimum value, solutions were also obtained using 18 and 24 support points. The point distributions near the top of the 
cylinder surface are shown in Figure 6.7 with the cloud support points highlighted in blue.

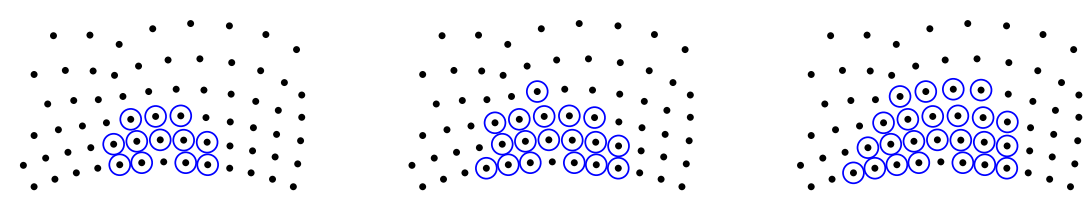
(a) $n s=12$
(b) $n s=18$
(c) $n s=24$

\section{Figure 6.7: Comparison of Local Cloud Configurations}

All three solutions were able to converge to the pre-determined iteration tolerance

of $10^{-6}$ in a predictable and smooth manner. The peak velocity on the upper surface was compared to the analytical solution of Van Dyke by calculating the relative error.[26] Table 6.2 summarizes the values obtained from each of the three cases. The error values for all three cases are on the order of $0.1 \%$ which indicates that the number of support points does not have a significant influence on the accuracy of the solution. What is interesting to note is that the error does not monotonically decrease with the number of support points. This may be due to the fact that there may be an optimal set of support points for each cloud that balances the effect of keeping the derivative approximation localized while also using an adequate amount of information from neighboring nodes. 
Table 6.2: Suction Peak Error Due to the Amount of Cloud Support Points

\begin{tabular}{ccc}
\hline \hline Number of Support Points & Suction Peak Velocity & Error (\%) \\
\hline Van Dyke & 2.259 & 0 \\
12 & 2.250 & 0.400 \\
18 & 2.253 & 0.293 \\
24 & 2.248 & 0.512 \\
\hline
\end{tabular}

\subsubsection{Influence of the Farfield Boundary}

The farfield boundaries used in this study were concentric circles with varying radii from the center of the cylinder. The node generation procedure follows the same method used by Zingg [69] who studied the farfield boundary effects on a NavierStokes solution for viscous airfoil flowfields. In this study he examined the boundary effects by first generating the mesh containing the outermost farfield boundary. Smaller bounded domains were then constructed by eliminating the cells that were outside of the new smaller farfield boundary. By reducing the problem domain in this manner the farfield boundary effects can be studied irrespective of the resolution of the grid.

The node generation process applied here involved creating the smallest farfield boundary first. All of the same baseline grid metrics were used except that the farfield boundary radius was chosen to be 10 instead of 20. A mesh was then grown off the boundary node distribution to the farfield boundary. Meshes with larger farfield boundaries were then grown off the original mesh. By growing the mesh in this manner, the resolution and distribution of nodes near the cylinder surface remained 
unaltered. Figure 6.8 shows the relative size of these three boundaries. The three different farfield boundary radii that were chosen for this study were 10, 20, and 40 .

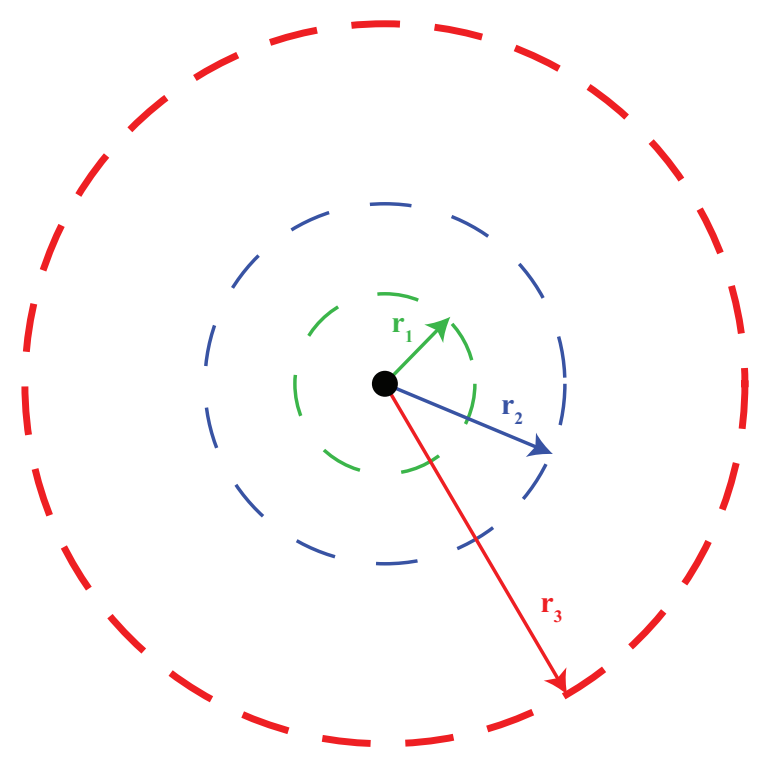

Figure 6.8: Outer Boundary Setup

The velocity at the upper suction peak was calculated for each of the converged solutions and was then compared to the analytical solution. Figure 6.9 shows that the relative error is proportional to the inverse of the square of the farfield boundary radius. If a linear fit is applied to this relationship, an extrapolated solution with a farfield boundary of radius 7.56 would result in a suction peak velocity error of $1.0 \%$. This behavior was expected because the Dirichlet boundary condition at the farfield forces the physics of the unbounded problem to be solved on a bounded domain. Therefore it would follow that the farfield boundary radius of 40 provides the best approximation to the unbounded physics of the problem. 


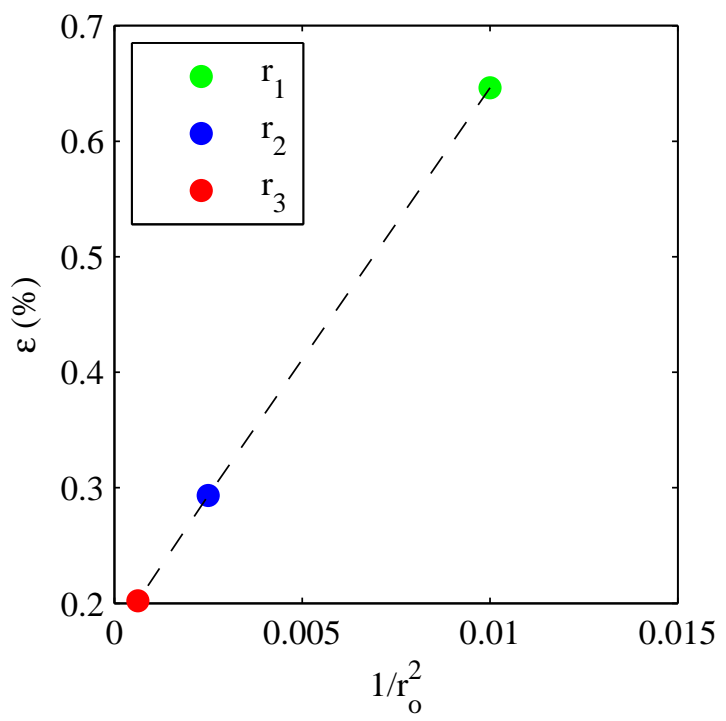

Figure 6.9: Suction Peak Error as a Function of the Farfield Radius

\subsubsection{Number of Boundary Nodes}

To study the effects of grid refinement, three node distributions with varying density were constructed. The baseline distribution (Distribution 2) was chosen to be the medium sized distribution. A denser grid (Distribution 1) was then generated from the baseline by distributing double the amount of nodes on the cylinder surface and then growing the domain nodes with the same grid metrics listed in Table 6.1. A coarser grid (Distribution 3) was also generated by distributing half the amount of nodes in the baseline mesh. The global effect of changing the number of nodes on the cylinder surface is that the total number $\left(N_{t}\right)$ of nodes changes. The local effect is that the mean distance $\left(d_{m}\right)$ of the points within the cloud surrounding the suction peak point changes. In order to quantify the change in mean distance between the medium sized grid and the coarse and fine grids, a ratio of these mean distances $(r)$ 
were calculated.

The coarsest grid has an order of magnitude larger error in predicting the suction peak velocity while the two finer meshes have errors on the same order of magnitude. This implies that the mean distance necessary for an accurate solution lies somewhere between the values listed in Table 6.3 for Distribution 3 and Distribution 2.

Table 6.3: Cylinder Mesh Refinement Data

\begin{tabular}{ccccccc}
\hline \hline Distribution & $N_{b}$ & $N_{t}$ & $d_{m}$ & $\mathrm{r}$ & $V_{\max }$ & $\epsilon(\%)$ \\
\hline 1 & 200 & 3012 & 0.033 & 0.47 & 2.26 & -0.13 \\
2 & 100 & 1971 & 0.069 & 1.00 & 2.25 & 0.29 \\
3 & 50 & 1320 & 0.147 & 2.13 & 2.16 & 4.47 \\
\hline
\end{tabular}

\subsubsection{Growth Ratio}

This study kept all of the mesh parameters in Table 6.1 constant except for the value of the cell growth ratio. In order to study the effects of this metric, the relative error in predicting the peak velocity was evaluated for meshes having growth ratios of 1.1, 1.2, and 1.4. In order to visualize the effect of the growth ratio, the nodes from Distribution 1 and Distribution 3 are shown side by side in Figure 6.10. As shown in Table 6.4, the growth ratio has a noticeable influence on the value of the suction peak velocity. Distribution 3 has an error that is an order of magnitude larger than Distribution 2 and an error that is two orders of magnitude larger than Distribution 1. While it was expected that the error gets smaller as the growth ratio also gets smaller it is apparent that the this grid metric can have a significant influence on the accuracy of the solution. 


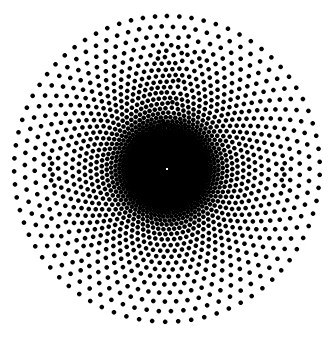

(a) Distribution 1

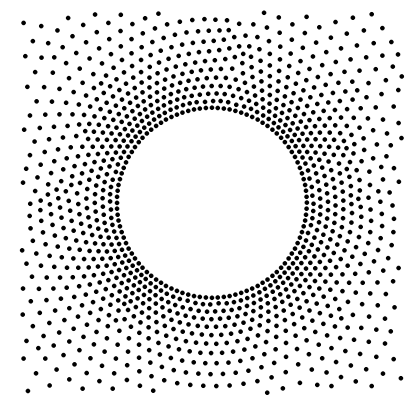

(c) Distribution 1 Near Surface

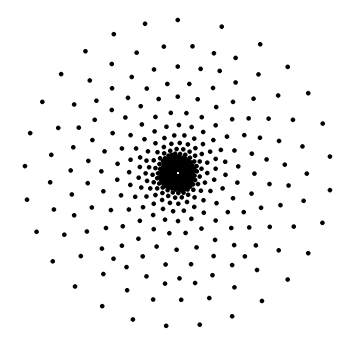

(b) Distribution 3

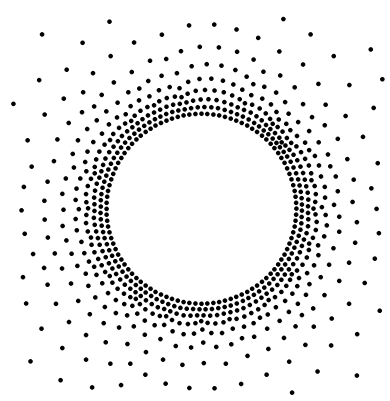

(d) Distribution 3 Near Surface

Figure 6.10: Comparison of Cylinder Node Distributions

Table 6.4: Cylinder Growth Ratio Study Data

\begin{tabular}{ccccc}
\hline \hline Distribution & Growth Ratio & $N_{t}$ & $V_{\max }$ & $\epsilon(\%)$ \\
\hline 1 & 1.1 & 4198 & 2.261 & -0.051 \\
2 & 1.2 & 1971 & 2.253 & 0.293 \\
3 & 1.4 & 1111 & 2.235 & 1.090 \\
\hline
\end{tabular}

\subsubsection{Variation with $M_{\infty}$}

The previous sections have shown the solution's dependence on various node distribution metrics for a single freestream condition; therefore, the robustness of the GFD method will also be tested by examining the solution accuracy for a wide range 
of freestream Mach numbers. Fortunately the Janzen-Rayleigh series for the peak velocity on the cylinder surface is valid for any subcritical Mach number.[26]

Van Dyke states that the original motivation for applying the Janzen-Rayleigh series was to resolve a topic known as the transonic controversy which postulates whether continuous shock free flow can be obtained for a given profile shape past the critical Mach number. Van Dyke states that the critical Mach number for the cylinder 0.39823780 to within one unit in the last figure. Numerical experiments conducted by Van Dyke also asserted that there may be shock free flow on the surface of the cylinder up to a freestream condition that is $1.1 \%$ of the critical Mach number but with the warning that this number may be reduced if more terms are added to the series.

Figure 6.11 shows the results from obtaining solutions with the baseline node distribution for freestream Mach numbers ranging from 0.1 to 0.421 . The predicted peak velocities match quite well for all of the Mach numbers within the subcritical range. Several cases were also ran at Mach numbers slightly above the critical value. It was observed that the solution diverged for a freestream Mach number above 0.421.

Figure 6.12 shows the overall pressure coefficient distribution for the case with a freestream Mach number of 0.4026 which corresponds to $1.1 \%$ above the critical value. The solution does not exhibit oscillatory or discontinuous behavior near the suction peak even though there is a small supersonic region. The results obtained from the GFD method may not be accurately predicting the true flowfield since it is 
not exactly known whether there actually exists shock-free flow, but this solution does demonstrate that the method is robust enough to reach an accurate and convergent solution that is right against the subcritical limit.

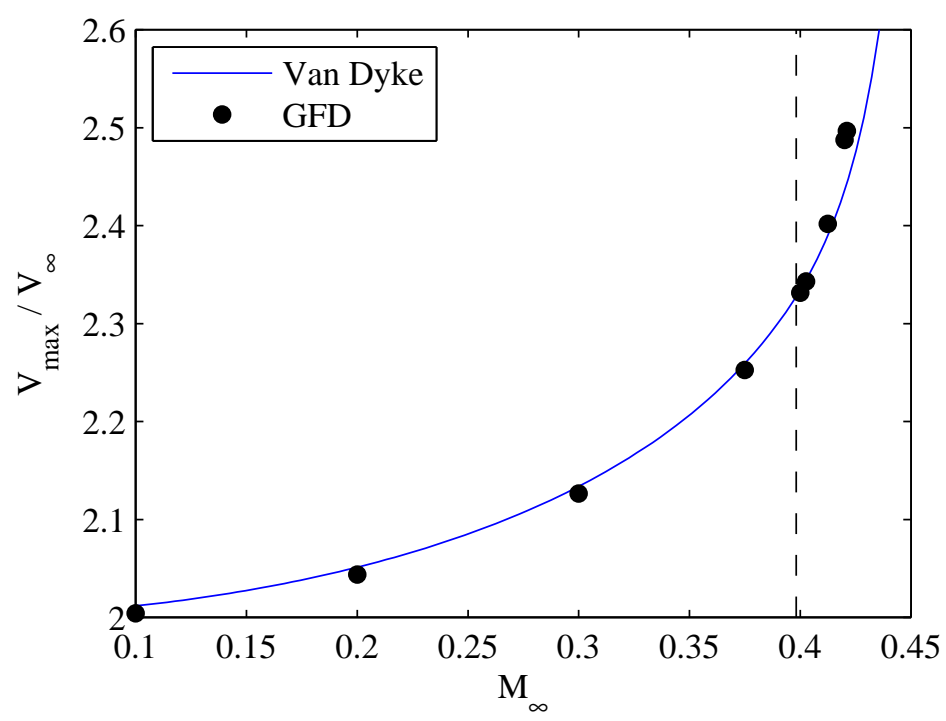

Figure 6.11: Comparison with Van Dyke for Varying $M_{\infty}$

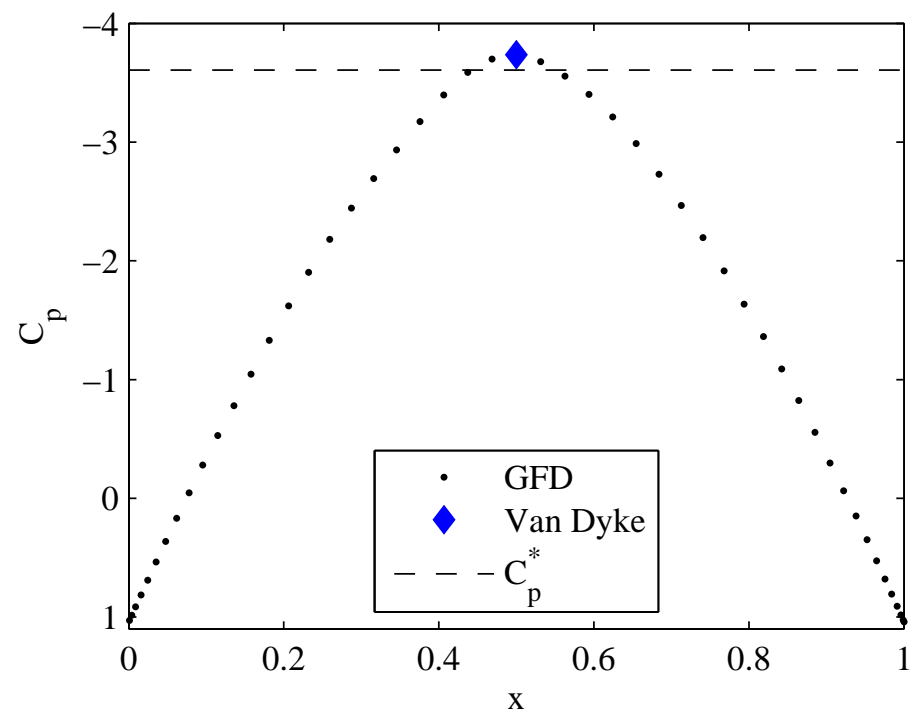

Figure 6.12: Supercritical Pressure Coefficient Distribution 
It has been shown how different grid and cloud metrics influence the accuracy of the solution for a circular cylinder at a 0.375 freestream Mach number. By analyzing the effect of these metrics some intuition has been gained on what type of node distribution can produce accurate results. Hopefully the knowledge gained from these studies has provided helpful suggestions that will guide researchers who would develop a meshless point generator. The next two sections of this chapter will focus on the solutions for a NACA 0012 airfoil in order to assess how well the generalized finite difference method can solve flows with features such as a sharp trailing edge and a discontinuous change of the potential across a wake cut plane.

\subsection{Non-Lifting NACA 0012}

The second case that was examined was the sub-critical flow over a NACA 0012 airfoil at a zero angle of attack. As with the case solved by the Dual Reciprocity Method, the freestream Mach number is 0.72 . The baseline node distribution for this study was generated in a similar manner as the circular cylinder with a circular farfield boundary as shown in Figures 6.13 and 6.14. The baseline grid has 200 intervals on the surface using the bell shaped scheme available in GAMBIT. The bell shaped scheme produces a normal distribution of the nodes centered at the half chord of the airfoil. A grading characteristic of 0.7 was chosen, which places a higher density of nodes at the leading and trailing edge. The farfield boundary was chosen to be a circle with radius 10 measured from the airfoil center. This differs from the previous 
problem because the compressibility effects are expected to be confined to a smaller area around the airfoil. The domain nodes were grown off the surface distribution to the farfield boundary using a 1.2 growth ratio.

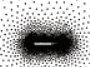

Figure 6.13: Baseline Airfoil Node Distribution

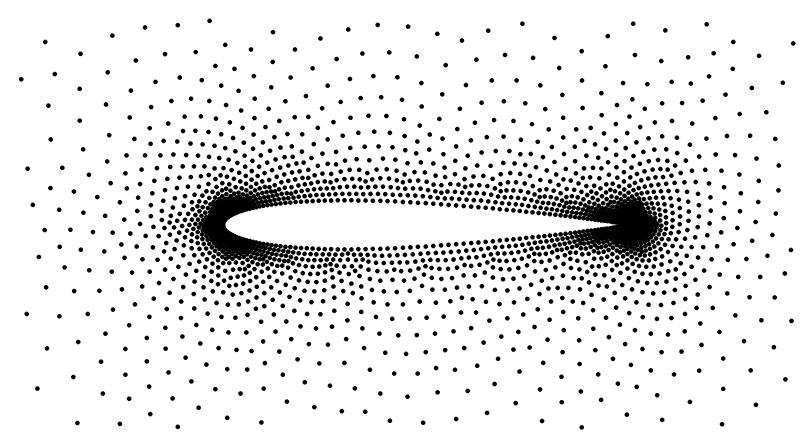

Figure 6.14: Close-Up View of Baseline Airfoil Node Distribution 
Table 6.5: NACA 0012 Baseline Distribution Metrics

\begin{tabular}{cc}
\hline \hline Number of Boundary Nodes & 200 \\
Farfield Boundary Radius & 10 \\
Cell Growth Ratio & 1.2 \\
Total Number of Nodes & 3288 \\
Number of Support Points & 18 \\
\hline
\end{tabular}

Recall from the description of the solution procedure in Chapter 5 that one of the flow variables that gets updated is the circulation $\Gamma$. It would follow that this value should be zero for a symmetric airfoil at zero angle of attack. One way to enforce this condition is to "hard code" that $\Gamma$ be zero for every iteration. For this study this rigid type of enforcement will not be applied. Instead the circulation will be calculated as usual in order to observe how well the method can predict the symmetry of the flowfield.

Since the airfoil is symmetric and this case is solving the problem for a zero angle of attack, it is expected that the lift coefficient should also be zero. According to the Kutta-Joukowski theorem, the lift per unit span $\left(L^{\prime}\right)$ can be expressed in terms of the circulation.

$$
L^{\prime}=\rho_{\infty} U_{\infty} \Gamma
$$

The lift per unit span can also be expressed in the more traditional way as a function of the dynamic pressure, surface area, and lift coefficient.

$$
L^{\prime}=\frac{1}{2} \rho_{\infty}\left|\mathbf{Q}_{\infty}\right|^{2} c C_{l}
$$

By combining these two equations, a simple expression for the lift coefficient as a 
function of the circulation can be obtained. For subsequent studies the lift coefficient will be calculated using Equation (6.3).

$$
C_{l}=\frac{2 \Gamma}{\left|\mathbf{Q}_{\infty}\right| c}
$$

The results from the converged solution on the baseline node distribution are presented in the next three figures. Figure 6.15 shows that the convergence of the solution is quite smooth and predictable. The predicted pressure coefficient distribution, as shown in Figure 6.16, matches very closely with the results obtained by Lock.[41] Figure 6.17 shows the value of $\frac{\partial \Phi}{\partial \mathbf{n}}$ on the surface of the cylinder in order to assess how well the Hermite type interpolation enforced the no normal flow boundary condition. The highest error is present near the leading edge with values on the order of $10^{-2}$. The error is higher than the results from the cylinder case which may be attributed to the fact that the curvature of the circle surface is constant whereas the curvature changes quite rapidly near the leading edge of an airfoil.

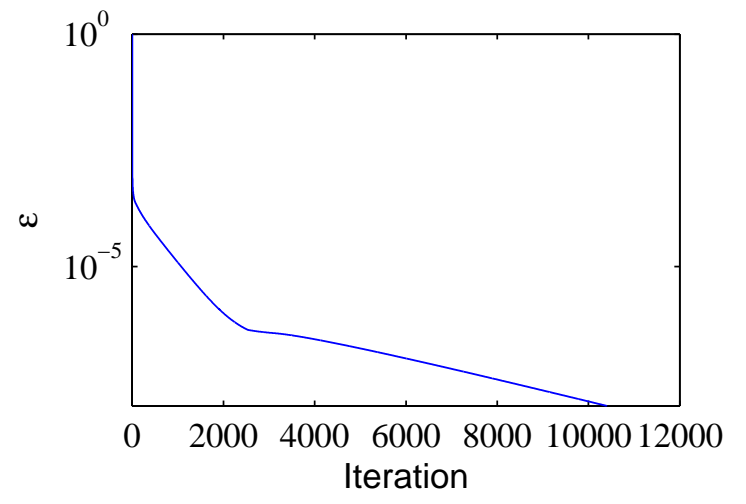

Figure 6.15: Non-Lifting Case Iteration History 


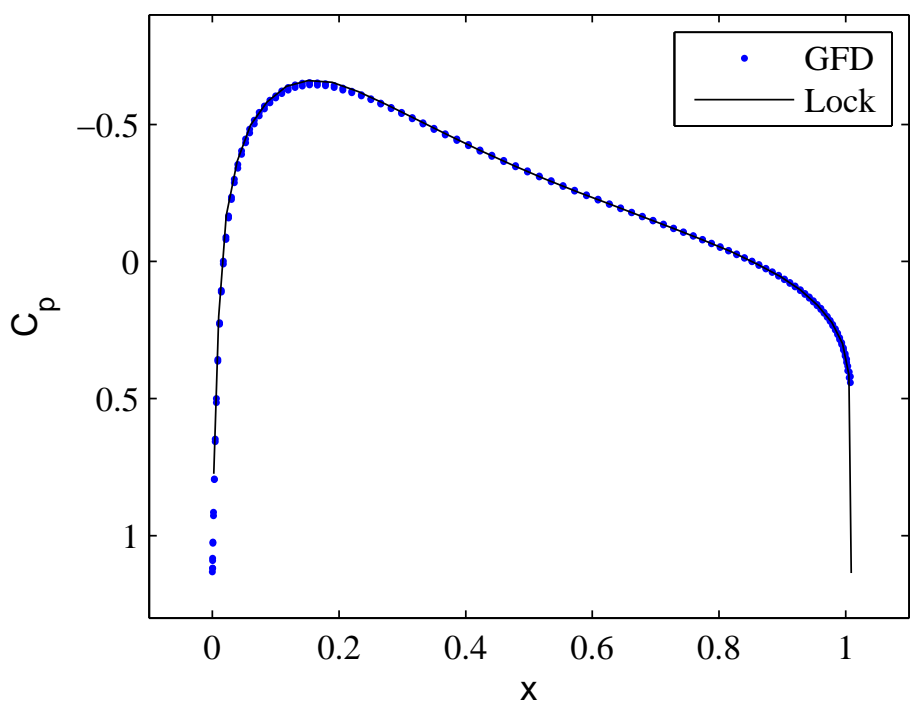

Figure 6.16: Pressure Coefficient Distribution for Non-Lifting Case $\left(M_{\infty}=0.72\right)$

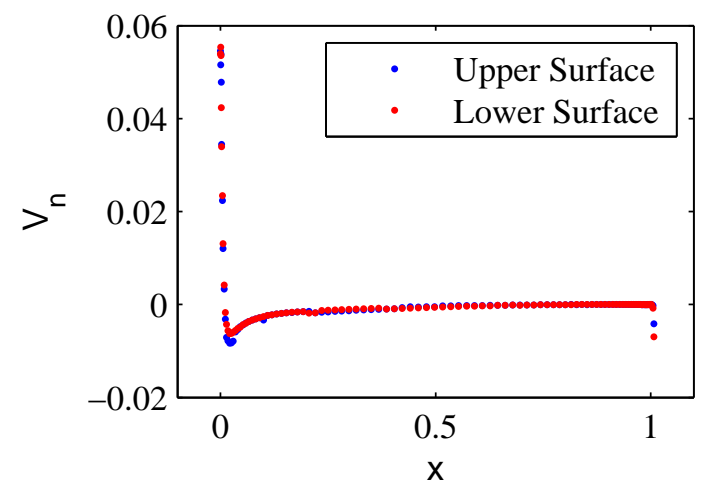

Figure 6.17: Normal Velocity Component for Non-Lifting Case

\subsubsection{Convergence Criteria}

Before the node distribution studies for this case could be conducted, it was important to determine when the iterative solution had converged to an asymptotic final answer. As with the cylinder problem, the first derivatives were initially calculated with third order accuracy and the second derivatives with second order accuracy by solving the least squares problem associated with an $l=3$ Taylor series. The data 
provided by Lock [41] indicates the local Mach number can reach values up to 0.98 . Therefore the meshless method needs to be capable of solving flows that are very close to reaching sonic conditions.

Applying the order of accuracy used in the cylinder case for approximating derivatives in the airfoil problem resulted in a converged solution exhibiting oscillatory behavior near the suction peak as shown in Figure 6.18. The oscillations could be attributed to the fact that the flow is nearly sonic near the suction peak and therefore the PDE is very close to transitioning from elliptic to hyperbolic. In the CFD community different types of flux limiters have been used to diminish the amount of oscillations near shocks. [4] These limiters approximate derivatives with high order in smooth regions of the flow and with low order approximations in the vicinity of discontinuities such as shocks.

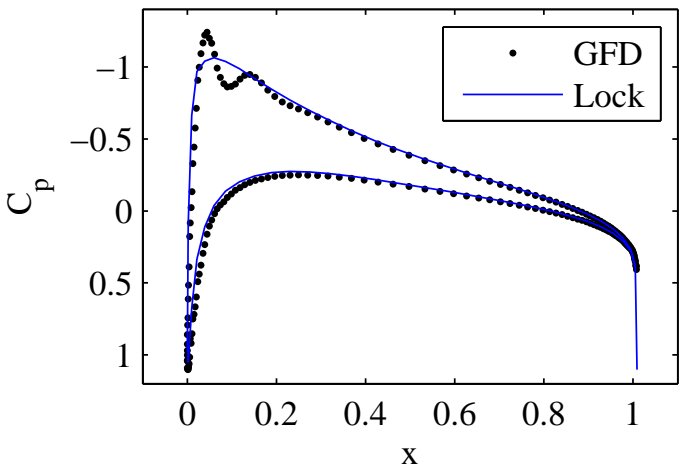

Figure 6.18: Oscillatory Pressure Coefficient Distribution

To mirror this type of behavior in the meshless solver, the calculation of the second derivatives remained unchanged but the derivative coefficients $w^{1 x}$ and $w^{1 y}$ were calculated using an $l=2$ Taylor series instead of the original implementation 
which used an $l=3$ series. When this modification was made, the oscillations were no longer present; therefore, for all subsequent solutions the estimates of both the first and second derivatives will be second order accurate.

A stable solution was obtained using this modification and Figure 6.19 shows how the value of the peak velocity changes as a function of the iteration error. It is apparent that the peak velocity shows minimal change once the iteration error reaches a value of approximately $10^{-6}$. Therefore the solution will be considered converged when the iteration error reaches this value. Figure 6.20 shows that there is a slight asymmetry in the pressure coefficient distribution between the upper and lower surface with the most notable difference located near the suction peak.

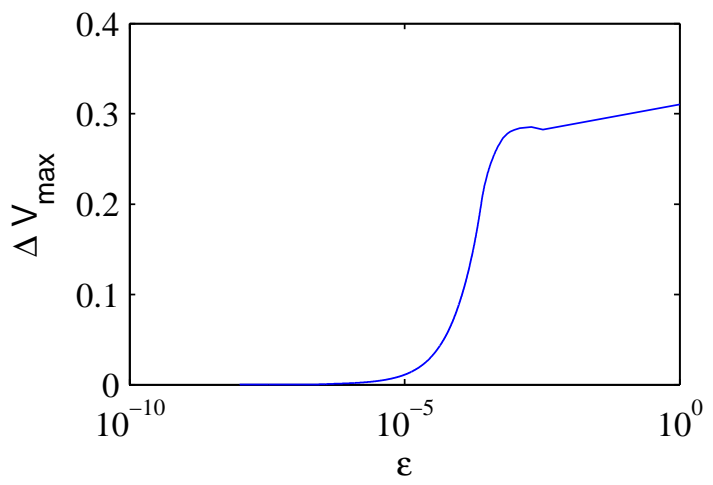

Figure 6.19: Maximum Velocity Evolution for Non-Lifting Case 


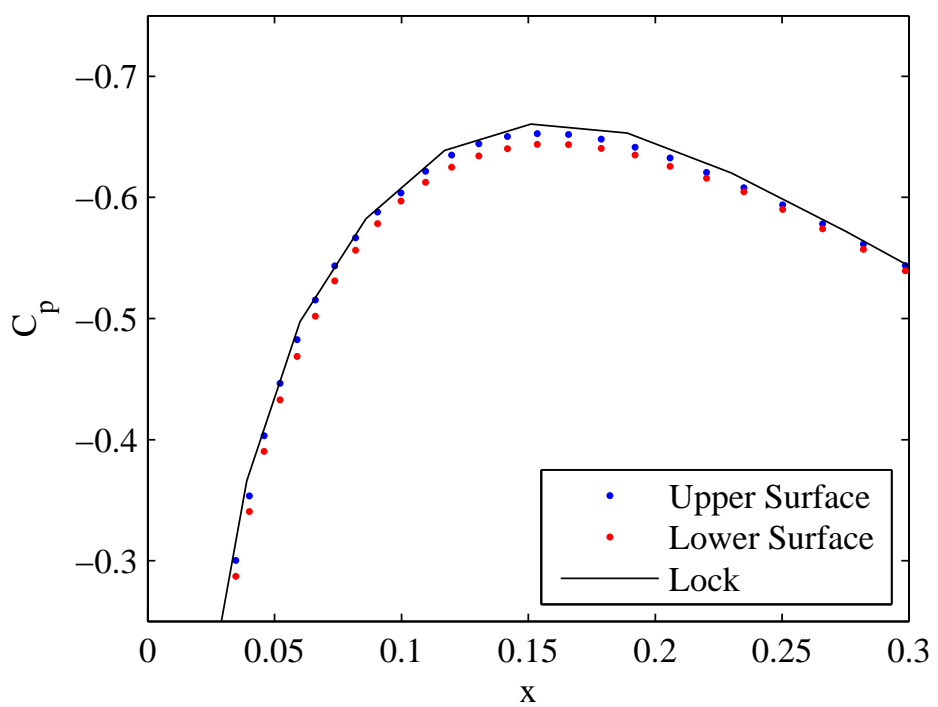

Figure 6.20: Comparison of $C_{p}$ on Upper and Lower Surface for Baseline Distribution

\subsubsection{Number of Support Points}

Since the truncated series used in the determination of the derivative coefficients $w^{2 x}$ and $w^{2 y}$ applies an $l=3$ Taylor series, the minimum amount of support points required for all of the clouds is $l\left(\frac{l+3}{2}\right)=9$. As with the circular cylinder problem, this amount of support points was chosen initially, but the solution was also unable to reach a convergent solution. It was determined that the minimum amount of support points that could adequately solve the baseline node distribution was 12 . To study the effects of increasing the number of support points from this minimum value, solutions were also obtained using 18 and 24 support nodes. The support points associated with the cloud generated for the point on the leading edge are shown in Figure 6.7.

The predicted pressure coefficient distributions for each of the three cases differed very slightly along most of the surface except near the suction peak. The pressure 


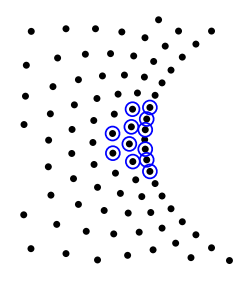

(a) $n s=12$

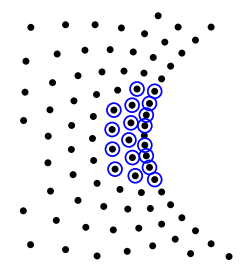

(b) $n s=18$

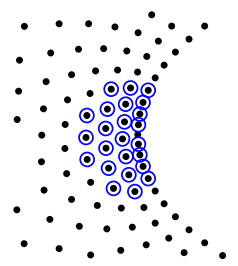

(c) $n s=24$

Figure 6.21: Comparison of Local Cloud Configurations at Leading Edge

coefficient values near this area are shown in Figure 6.22. The accuracy of the solution degrades as the number of support points increases. This behavior indicates that the approximation needs to be as localized as possible in order to yield the most accurate results.

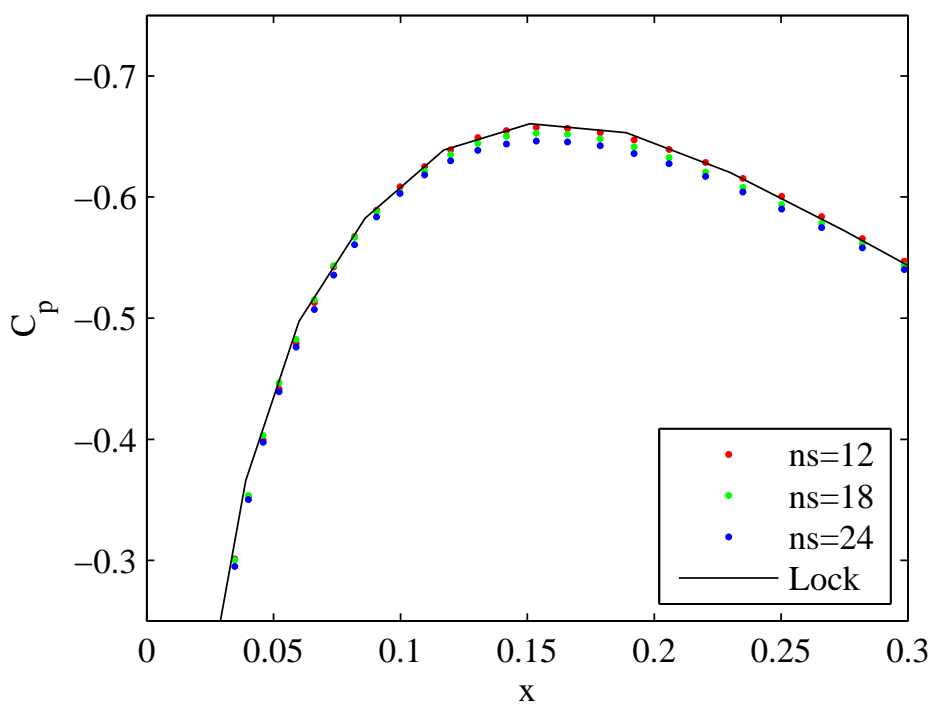

Figure 6.22: Pressure Coefficient Near Suction Peak as a Function of the Number of Cloud Support Points

The predicted lift coefficient was also calculated for the three cases. As shown in Table 6.6 the predicted values are quite small which indicates that the asymmetry in 
the flowfield is not significant. As with the predicted pressure coefficient distribution the solution with twelve cloud support points yielded the most accurate result.

Table 6.6: Lift Coefficient as a Function of the Number of Support Points

\begin{tabular}{cc}
\hline \hline Number of Support Points & Lift Coefficient \\
\hline 12 & 0.0022 \\
18 & 0.0050 \\
24 & 0.0056 \\
\hline
\end{tabular}

\subsubsection{Influence of the Farfield Boundary}

The influence of the farfield boundary on the predicted pressure coefficient distribution and lift coefficient was also studied. The smallest farfield boundary has a radius of 5 and the largest boundary has a radius of 20. Table 6.7 shows that the location of the farfield boundary does not have a significant impact on the prediction of the lift coefficient because this grid characteristic does not have a significant influence on the asymmetry of nodes near the airfoil surface.

Table 6.7: Lift Coefficient as a function of the Farfield Radius

\begin{tabular}{cc}
\hline \hline Farfield Radius & Lift Coefficient \\
\hline 5 & 0.0029 \\
10 & 0.0050 \\
20 & 0.0030 \\
\hline
\end{tabular}

The influence the placement of the farfield boundary has on the predicted pressure coefficient distribution is most readily apparent near the suction peak. Figure 6.23 shows the pressure coefficient values on the upper surface of the airfoil for the three different cases. All three solutions under predict the magnitude of the suction peak 
with the smallest farfield boundary case $\left(r_{o b}=5\right)$ having the largest error whereas the other two solutions $\left(r_{o b}=10\right.$ and $\left.r_{o b}=20\right)$ do not have as much of a noticeable difference.

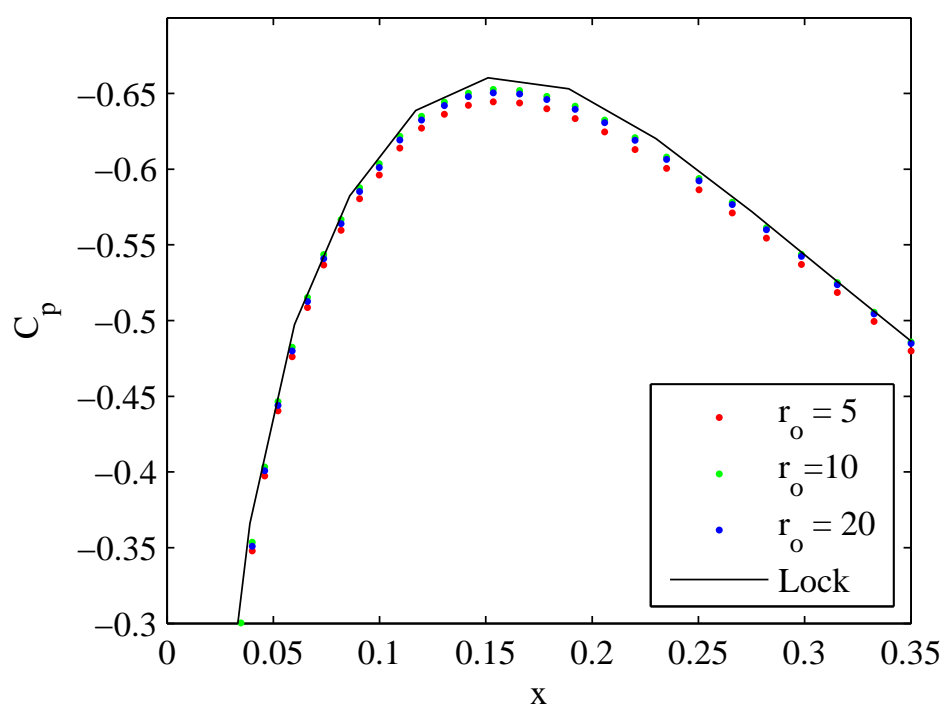

Figure 6.23: Comparison of $C_{p}$ on Upper Surface for Different Farfield Radii

\subsubsection{Number of Boundary Points}

To study the effects of node resolution the results from distributions with 100 and 400 total nodes on the airfoil surface were compared to the solution from the baseline node distribution. The nodes were distributed in the same manner as the baseline with a bell shaped distribution with a grading characteristic of 0.7 . Figure 6.24 shows that, as expected, the accuracy of the solution increases as the density of nodes also increases. 


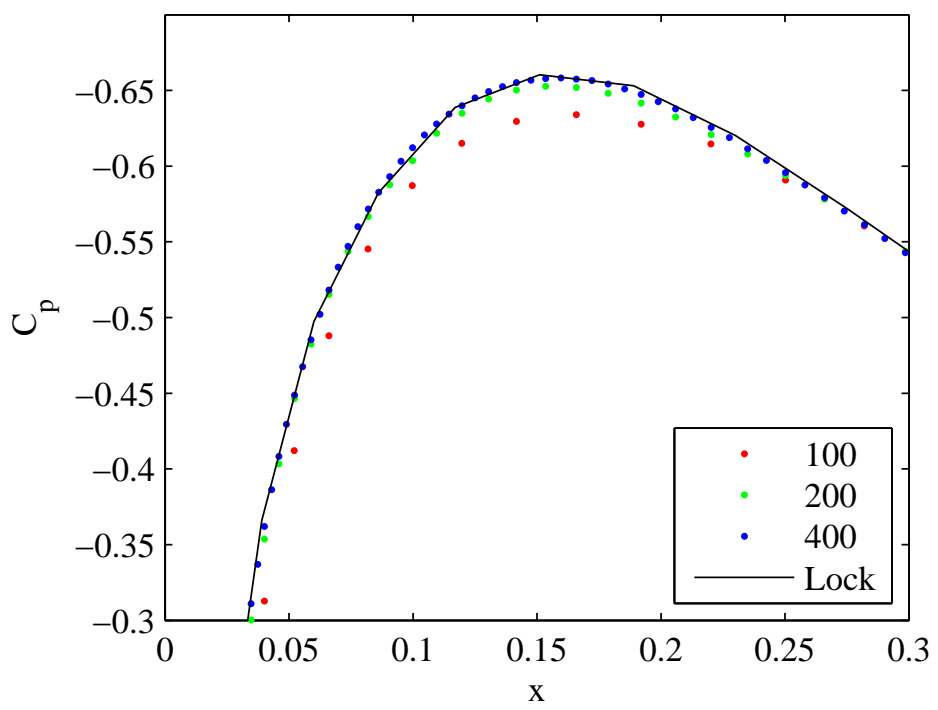

Figure 6.24: Comparison of Upper Surface $C_{p}$

The predicted lift coefficient does not monotonically decrease as the resolution increases as might be expected. This may be due to the fact that the asymmetries in the node distribution generated in GAMBIT do not diminish as the resolution gets higher. The ratio calculated in the cylinder results section which compares the change in mean distance from the baseline distribution is also reported for this case in Table 6.8 which shows that the doubling or halving of the number of nodes on the airfoils surface also roughly corresponds to the doubling or halving of the mean distance for support points associated with the cloud generated around the surface point on the leading edge of the airfoil. 
Table 6.8: Mean Distances for Clouds Associated with the Leading Edge Point

\begin{tabular}{ccccc}
\hline \hline$N_{b}$ & $N_{t}$ & $d_{m} \times 10^{-3}$ & $\mathrm{r}$ & $c_{l}$ \\
\hline 100 & 2357 & 9.457 & 1.88 & 0.0037 \\
200 & 3288 & 5.029 & 1 & 0.0050 \\
400 & 5005 & 2.815 & 0.56 & 0.0029 \\
\hline
\end{tabular}

\subsubsection{Growth Ratio}

The growth ratio of cells was also examined to study its affect on both predicting the pressure coefficient distribution and the lift coefficient. A fine density distribution was generated by growing a grid with a 1.1 growth ratio and a coarse distribution was generated by applying a growth ratio of 1.4. These two distributions are shown in Figure 6.25. Table 6.9 shows that the growth ratio does not have a predictable influence on the accuracy of calculating the lift coefficient.

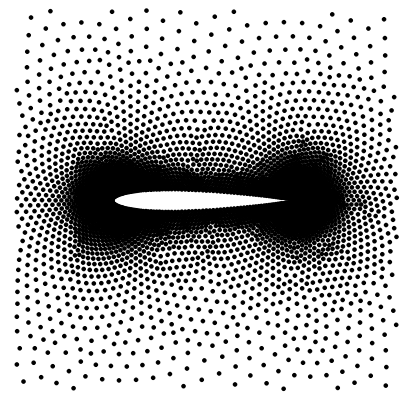

(a) Distribution 1 Near Surface

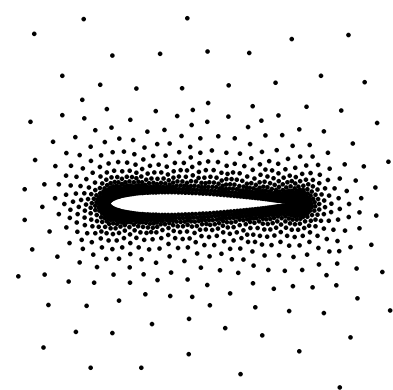

(b) Distribution 3 Near Surface

Figure 6.25: Comparison of Node Distributions on Airfoil 
Table 6.9: Airfoil Ratio Study Data

\begin{tabular}{ccc}
\hline \hline Growth Ratio & $N_{t}$ & $c_{l}$ \\
\hline 1.1 & 7108 & 0.0017 \\
1.2 & 3195 & 0.0050 \\
1.4 & 1723 & 0.0007 \\
\hline
\end{tabular}

The growth ratio does have a noticeable influence on the prediction of the pressure coefficient distribution. As with the other cases the disparity between the GFD solution and the data from Lock is most significant near the suction peak. It is clear from Figure 6.26 that a growth ratio of 1.4 results in a distribution that is too coarse, but the solutions for the growth ratio of 1.1 and 1.2 node distributions yield very similar results. Therefore it would seem that a growth ratio of 1.2 can adequately solve the problem and not necessitate the solution on a denser and more computationally expensive distribution.

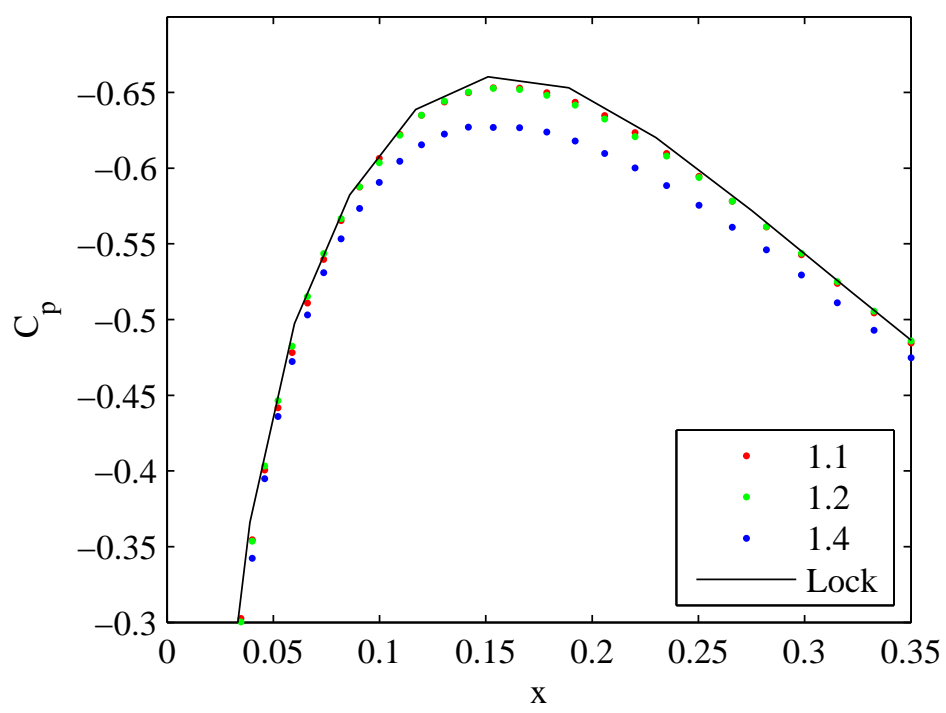

Figure 6.26: Comparison of Upper Surface $C_{p}$ as Function of Growth Ratio 


\subsection{Lifting NACA 0012}

The last case that was examined was the flow over the NACA 0012 airfoil with a freestream Mach number of 0.63 and an angle of attack of $2^{\circ}$. These freestream conditions were chosen because the flowfield is still sub-critical and the benchmark results are available in the AGARD report written by Lock [41]. Studies that were conducted in the previous section were not conducted here because the geometry and node distribution is the same with the only difference being the freestream conditions. The results are shown for the solution of the full potential equation using the baseline node distribution from the previous section.

As with the non-lifting case the convergence history is quite smooth and predictable yet it took longer for the lifting-case to converge. This could be attributed to the fact that there are more circulation value updates needed for this case.

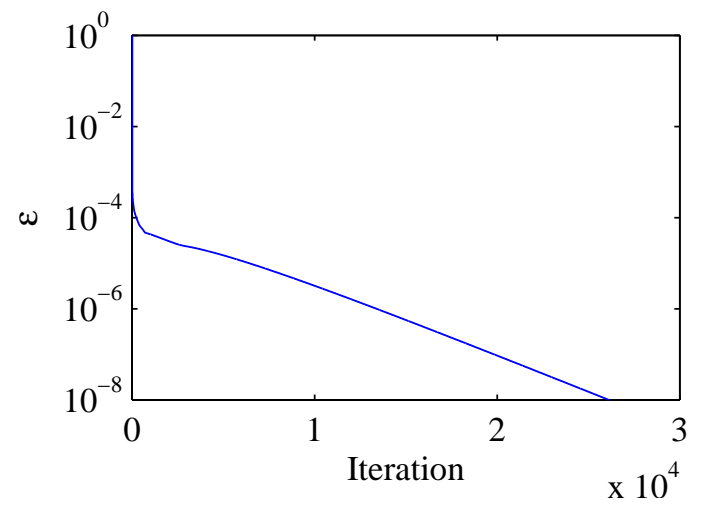

Figure 6.27: Lifting Case Iteration History

Two different flow features were tracked during the iterative process in order to assess when the solution was converged. As with the non-lifting airfoil the maximum 
velocity was calculated for each iteration. The lift coefficient was also evaluated at each iteration in order to provide an indication for how the farfield boundary condition evolved. Both of these flow features reached an asymptotic final result around an iteration error of approximately $10^{-6}$ which is the same value it occurred for the nonlifting case. The converged value of the lift coefficient calculated by Equation (6.3) was 0.332 which matches very closely with the reported value of 0.335 given by Lock [41].

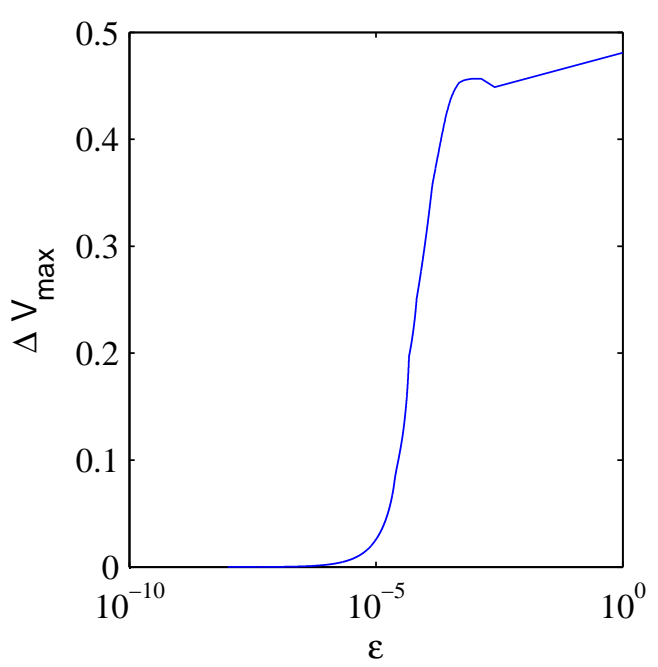

(a) Maximum Velocity

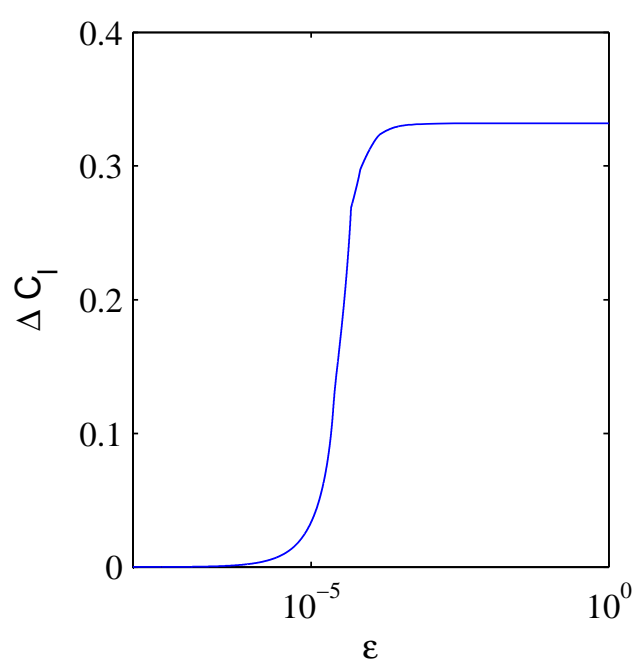

(b) Lift Coefficient

Figure 6.28: Evolution of Flow Characteristics for Lifting Airfoil Case

The pressure coefficient distribution matches quite well to the results provided by Lock. As with the non-lifting case, the largest disparity between the two solutions is located near the suction peak. Lock reports that the local Mach number reaches a maximum value of 0.983 which means that this solution can handle flow cases with nearly sonic conditions. 


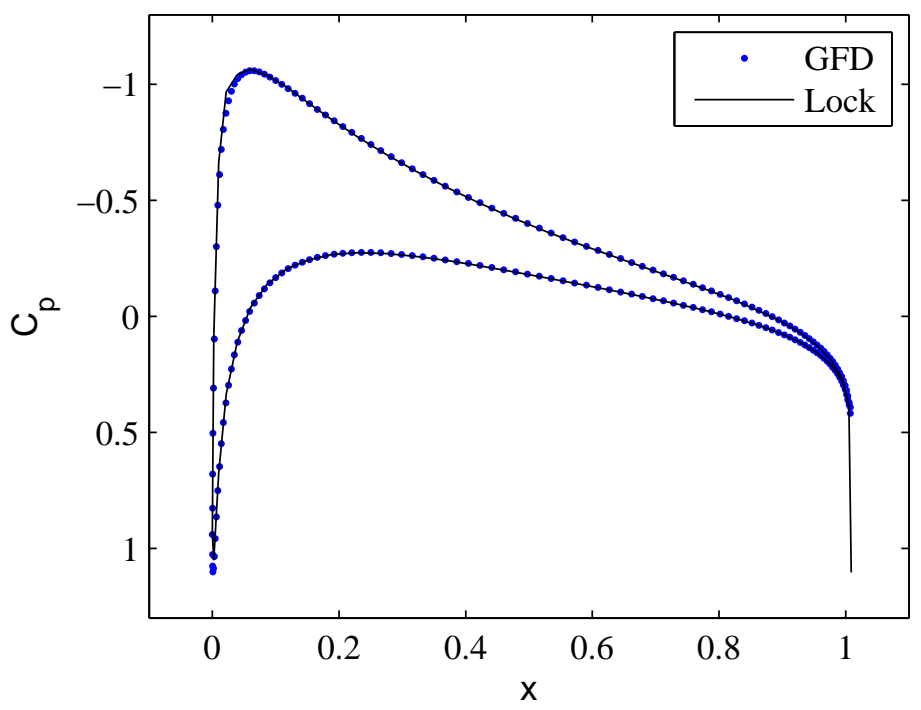

Figure 6.29: Pressure Coefficient Distribution for Lifting Case

The error in enforcing the no normal flow boundary condition is comparable to the results from the non-lifting case with the maximum error encountered near the leading edge. Unlike the non-lifting case, the asymmetry in the flow results in a negative normal velocity on the upper surface and a positive normal velocity on the lower surface near the leading edge.

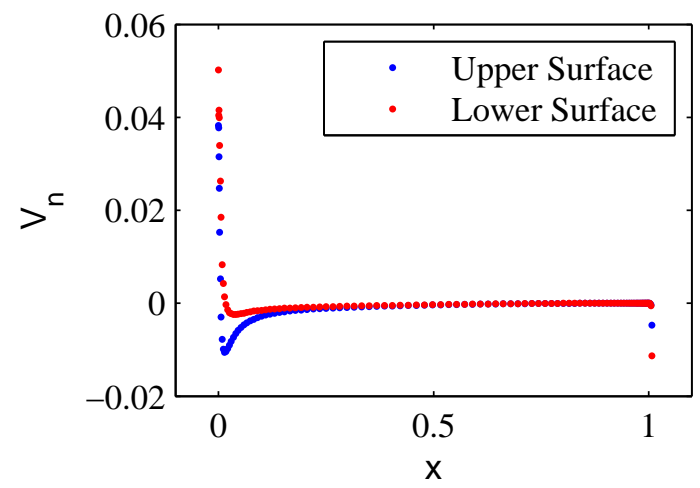

Figure 6.30: Normal Velocity Component for Lifting Case 
This chapter has confirmed that the generalized finite difference method can adequately solve the full potential equation for both lifting and non-lifting flows. "Grid" convergence studies were conducted in order to assess how different grid and support cloud metrics influence the accuracy of the solution. These studies were also performed in order to establish any "rules of thumb" that can be applied for any meshless node generator that may be developed in the future. These studies showed that the baseline distributions possessed the adequate density of nodes for both the cylinder and NACA 0012 cases. The observations made on the adequate number of cloud support points imply that a conservatively high value of support clouds ensure stability while not significantly degrading the accuracy of the solutions. The generalized finite difference method has proved to be a more robust and design friendly aerodynamic analysis tool when compared to the dual reciprocity method. A more detailed comparison of the two methods will be provided in the next chapter. 


\section{Chapter 7}

\section{Final Remarks}

Two different meshless methods were developed for solving the full potential equation as applied to external aerodynamic problems. While both methods were shown to adequately solve for the sub-critical compressible flowfield about a circular cylinder and NACA 0012 airfoil, each method presented a unique set of advantages and challenges. The next section will outline some research contributions that seem to be unique to this thesis. Secondly, comparisons will be drawn between the two methods by highlighting the pros and cons of using one technique over the other. Lastly, some suggestions for future research that can be undertaken to bring the code to a higher level of maturity will be addressed.

\subsection{Unique Contributions}

The research conducted for this thesis brought forth some unique contributions to the study of meshless methods to solve aerodynamic problems. This section will highlight some of the unique aspects of this research in order to show how it ties into 
the overall study of meshless methods.

The RBF chosen for the DRM solutions inherently satisfied the regularity conditions associated with the farfield boundary. The literature search conducted has not yielded any accounts of researchers applying this type of RBF to external aerodynamic problems. The freedom from modeling the farfield boundary allowed for the reduction in the number of domain points necessary to adequately solve the problem.

While the solution of compressible potential flows has been achieved by other authors by applying the Dual Reciprocity Method, the literature search for methods that apply a differential approach such as the GFD method did not yield any publications that address the solution of the full potential equation. There has been much research done for solving the Euler and Navier-Stokes equations using the GFD along with other differential methods, but it seems that this thesis is the first application of the Generalized Finite Difference method for the solution of compressible potential flows.

Lastly the enforcement of the Neumann boundary condition by applying a Hermitetype interpolation seems to not have been implemented in other publications. While this method does not enforce the no normal flow boundary condition exactly, it is more simple to implement than the commonly used ghost node method since no reflected nodes need to be generated. 


\subsection{Comparison of the Two Methods}

This section will first discuss the differences between the two methods in order to highlight the advantages that one technique may have over another. Lastly the similarities will be discussed in terms of the common challenges each method shares so that it can be shown in the subsequent section how they can be overcome with the suggestion of future research areas.

The Dual Reciprocity Method which is based off solving the problem in integral form proved to be an impractical solution technique due to the need for a priori knowledge of the correct solution in order to tune the free parameter necessary for the radial basis function approximation. If a consistent non-solution based procedure could be developed to determine this parameter the DRM could prove to be a very valuable tool since nodes would only need to be scattered where compressibility is dominant which leads to a faster convergence rate.

The generalized finite difference method works the same way as a standard finite difference method but without the need for connectivity data. The GFD method provides a significant advantage over the DRM due to the fact that no a priori knowledge of the solution is necessary to converge to an accurate result. A factor associated with the method that may be classified as empirical is the selection of the amount of support points. While it seems that there is no upper limit on the amount of points that may be chosen for a stable solution there does seem to be a lower limit. The example problems from the previous chapter suggest that the minimum amount of 
cloud support points is slightly higher than the amount suggested by the number of terms kept in the truncated Taylor series.

While the GFD method is more robust than the DRM it comes with the consequence of having to explicitly model the farfield boundary conditions. The RBF approximation used in the DRM procedure satisfied the regularity conditions which eliminated the need to model the farfield boundary. The GFD method needs to model the farfield boundary to correctly model both the freestream conditions and the circulation for lifting flows. As a consequence, more domain nodes need to be scattered since the farfield boundary needs to be placed at a sufficiently far distance form the surface of interest. The higher number of domain nodes along with the need to update the circulation value causes the GFD method to take a significantly larger number of iterations to converge.

Both methods solved the continuity equation in its non-conservative form. Solving the governing equation in this form did not turn out to be a significant source of error since the flowfield was smooth and continuous because no shocks were present. Theurer et. al. [7] observed that there was no significant difference between using the conservative or non-conservative form which suggests that it may only become important when solving problems with shocks where the discontinuous change in the flowfield needs to be captured accurately.

The presentation of the Dual Reciprocity Method was also more complicated when compared to the generalized finite difference method in terms of the mathematical 
principles applied. The implementation of the boundary integral equations derived for the DRM are not as conceptually tangible as the derivative technique of the GFD method. It was also shown in this thesis that some rules of thumb similar to conventional CFD techniques can be stated for the GFD method. It was the goal of this thesis to develop a tool where the designer does not need to be an expert on the subject of the solution method. Therefore the GFD method lends itself to being a more intuitive tool to use by the designer.

It was also confirmed that the generalized finite difference method shares similar discretization error properties to a standard finite difference scheme. The method developed in this thesis specifically established a second order accurate solver which is an important property a numerical solution needs to exhibit in order for it to be worthy of publication in journals such as the AIAA Journal of Aircraft[68] and ASME Journal of Fluids Engineering.[67] It is also important to note that the interpolation properties of a least squares fit of a Taylor series is better understood than the properties of a radial basis function. Much work still needs to be done in the formal mathematical analysis of the error properties of a radial basis function before it can prove to be a robust method of interpolation.

It has also been demonstrated by other authors $[57,70]$ that the Generalized Finite Difference method can be formulated to solve hyperbolic problems with upwinding and artificial dissipation. This kind of research strengthens the argument for using the GFD method to solve problems in the transonic regime using the full potential 
equation since it has already been shown to work by solving the Euler equations.

There is also a subtle difference in terms of how meshless the two techniques can be classified. The Dual Reciprocity Method still works off of a panel code framework which requires a surface mesh to correctly evaluate the boundary integral equations. Although the discretization of the surface geometry is still required, the DRM can still be considered a meshless scheme since it still reduces the dimensionality of the domain type methods such as finite volume and field panel methods that require a domain grid to evaluate the source term introduced by compressibility. On the other hand, the GFD method does not even require a surface mesh to solve the full potential equation. The only geometry information required by the solver is the classification of whether a node belongs to a boundary or to the domain and the direction of the unit normal vector for all the associated boundary nodes.

\subsection{Future Work}

The elephant in the room in terms of the work that needs to be done to develop a truly meshless aerodynamic design tool is the easy and fast implementation of a meshless node generator. Much of the meshless research done not only by this author but by other researchers in the meshless method community have used standard grid generators to establish a baseline node distribution in order to generate the domain nodes necessary for a meshless method. For a truly meshless design tool to come to fruition, a fast and easy technique for generating these node distributions without the 
need for a mesh generator is absolutely necessary. Promising work has been developed in this area by Löhner and Oñate $[60,61]$ who developed an advancing front point generation technique.

This seems like a very daunting task since there has not been much research done in this area. But meshless methods can also establish a different niche for themselves other than their use as a completely meshless solution technique. Research has also been done in terms of using a hybrid mesh and meshless technique. One such example is the work done by Carolina et. al. [71] and Luo et. al. [72, 73] to develop a hybrid Cartesian-meshless technique. The work done by Carolina et. al. used a standard Cartesian grid to solve the governing equations everywhere except in the vicinity of cut cells that intersect the geometry surface. In this region a radial basis function technique was used to enforce the boundary conditions. The work of Luo et. al. employs a similar procedure but uses a least squares Taylor series instead of an RBF to enforce the boundary conditions.

If one would want to focus specifically on improving the solver itself, there are multiple topics that can be explored further. First off the Neumann boundary condition enforcement used in the GFD method employed in this thesis performed quite well in areas of small or constant curvature yet there was still signs of the flow "leaking" through the surface, especially in the vicinity of an airfoil leading edge. The lower accuracy in this region may be attributed to a variety of reasons. It may be that the resolution of nodes in this region was not sufficient to capture both the rapid change 
in velocity and curvature. It could also be attributed to the weighting procedure employed in the least squares Taylor series fit. The inverse distance weighting procedure was used merely because it makes sense to add more bias to points located closer to the cloud center. It may be also necessary to add a higher bias to points located on the problem boundary which could result in a better approximation of the no normal flow boundary condition.

Another solver based topic which needs exploration is the extension of the flow regime that this method can adequately solve. As mentioned previously much work has been done in terms of developing solvers that can adequately capture flow features such as shocks using meshless upwinding techniques. These techniques were specifically applied to solving the Euler equations but in principle it could also be applied to solving full potential flows in the transonic regime. While it is true that the full potential equation can only describe isentropic flows, the non-isentropic effect of a shock could still be adequately handled if artificial dissipation is employed.[74]

The Taylor series technique described in this thesis revolved around solving twodimensional flows. One obvious research area that can be explored is the extension of the technique to solve three-dimensional problems. The truncated Taylor series used for the least squares fit could easily be extended to a third dimension to discretize a PDE about a three dimensional cloud. As observed in the previous chapter, the update of the farfield boundary condition due to the evolution of the circulation as the solution iterated caused a higher number of iterations necessary for a converged 
solution. This issue can be mitigated by initializing the meshless solver with the incompressible solution provided by a standard 3D panel method. Panel methods such as PMARC [75] use wake time stepping to establish a better approximation for the circulation by allowing the wake geometry to change. The converged solution from a code such as PMARC has the potential to reduce the iterations needed to arrive at a converged compressible solution from a meshless solver.

The future work outlined in this section is just a small example of the different directions this research can take. The development of meshless techniques may still be considered in its infancy yet the advances made over the last couple of decades shows that it can share a proper role with the more standard CFD tools used today. It is my hope that this thesis has contributed to this research area and that it will spark future researchers interests in developing faster and more efficient aerodynamic design tools. 


\section{References}

[1] Dawes, W. N., Dhanasekaran, P. C., Demargne, A. J., Kellar, W. P., and Savill, A. M., "Reducing Bottlenecks in the CAD-to-Mesh-to-Solution Cycle Time to Allow CFD to Participate in Design," Journal of Turbomachinery, Vol. 123, 2001, pp. 552-557.

[2] Jameson, A. and Vassberg, J. C., "Computational Fluid Dynamics for Aerodynamic Design: Its Current and Future Impact," 39th AIAA Aerospace Sciences Meeting and Exhibit, AIAA-2001-538, 2001.

[3] Anderson, J. D., Fundamentals of Aerodynamics, McGraw Hill, New York, 4th ed., 2007.

[4] Tannehill, J. C., Anderson, D. A., and Pletcher, R. H., Computational Fluid Mechanics and Heat Transfer, Taylor and Francis, 2nd ed., 1997.

[5] John, J. E. and Keith, T. G., Gas Dynamics, Pearson Prentice Hall, 3rd ed., 2006. 
[6] Morino, L. and Iemma, U., "Boundary Integral Equations and Conservative Dissipation Schemes for Full Potential Transonic Flows," Computational Mechanics, Vol. 13, 1993, pp. 90-99.

[7] Theurer, R. and Wagner, S., "Inviscid Transonic Flow Computation Using the Dual Reciprocity Method," Boundary Element Method Technology, Vol. 11, 1996, pp. $13-22$.

[8] Katz, J. and Plotkin, A., Low-Speed Aerodynamics, Cambridge University Press, Cambridge, MA, 2nd ed., 2001.

[9] Holst, T. L., "Transonic Flow Computations Using Nonlinear Potential Methods," Progress in Aerospace Sciences, Vol. 36, 2000, pp. 1-61.

[10] Kellog, O. D., Foundations of Potential Theory, Dover Publications, 1953.

[11] Hess, J. L. and Smith, A. M. O., "Calculation of Non-Lifting Potential Flow About Arbitrary Three-Dimensional Bodies," Tech. Rep. ES 40622, Douglas Aircraft, Longh Beach, CA, 1962.

[12] Henne, P. A., editor, Applied Computational Aerodynamics, Vol. 125 of Progress in Astronautics and Aeronautics, Washington, D.C., 1990, AIAA.

[13] Brebbia, C. A. and Butterfield, R. A., "Formal Equivalence of Direct and Indirect Boundary Element Methods," Applied Mathematical Modelling, Vol. 2, 1978, pp. $132-134$. 
[14] Brebbia, C. A., Telles, J. C. F., and Wrobel, L. C., Boundary Element Techniques: Theory and Applications in Engineering, Springer-Verlag, 1984.

[15] Brebbia, C. A., The Boundary Element Method for Engineers, John Wiley and Sons, New York, 1978.

[16] Theurer, R., Ein erwitiertes Randelementverfahren zur Berckichtigung nichtlinear Effekte in der Aerodynamik, Master's thesis, Universitt Stuttgart, Dusseldorf, 1998.

[17] Uhl, B., Ostertag, J., Guidati, G., and Wagner, S., "Application of the Dual Reciprocity Method to Three Dimensional Compressible Flows Governed by the Full Potential Equation," 37th AIAA Aerospace Sciences Meeting and Exhibit, AIAA-99-0168, 1999.

[18] Nardini, D. and Brebbia, C. A., "A New Approach to Free Vibration Analysis Using Boundary Elements," Boundary Element Methods in Engineering, Springer-Verlag, 1982.

[19] Partridge, P. W., Brebbia, C. A., and Wrobel, L. C., The Dual Reciprocity Boundary Element Method, Computational Mechanics Publications, Billerica MA, 1992. 
[20] Partridge, P. W. and Brebbia, C. A., "The Dual Reciprocity Method," Formulations in Boundary Elements, edited by M. H. Aliabadi and C. A. Brebbia, Computational Mechanics Publications, Billerca MA, 1993, pp. 31-75.

[21] Zhang, H. L., Rottgermann, A., and Wagner, S., "Field Panel Method with Grid Stretching Technique for Solving Transonic Potential Flow Around Arbitrary Airfoils," Computational Mechanics, Vol. 15, 1995, pp. 384-393.

[22] Golberg, M. A. and Chen, C. S., "A Bibliography on Radial Basis Function Approximation," Boundary Elements Communications, Vol. 7, No. 4, 1996, pp. 155163.

[23] Partridge, P. W. and Brebbia, C. A., "Computer Implementation of the BEM Dual Reciprocity Method for the Solution of General Field Equations," Communications in Applied Numerical Methods, Vol. 6, 1990, pp. 83-92.

[24] Loeffler, C. F. and Mansur, W. J., "Dual Reciprocity Boundary Element Formulation for Potential Problems in Infinite Domains," Boundary Elements X, 1988.

[25] Zhu, S. and Zhang, Y., "Solving General Field Equations in Infinite Domains with Dual Reciprocity Boundary Element Method," Engineering Analysis with Boundary Elements, Vol. 12, 1993, pp. 241-250. 
[26] Van Dyke, M., "Long Series in Mechanics: Janzen-Rayleigh Expansion for a Circle," Meccanica, Vol. 33, 1998, pp. 517-522.

[27] German, B. J., A Riemannian Geometric Mapping Technique for Identifying Incompressible Equivalents to Subsonic Potential Flows, Ph.D. thesis, Georgia Institiute of Technology, 2007.

[28] German, B. J., "Laplacian Equivalents to Subsonic Potential Flows," AIAA Journal, Vol. 47, No. 1, 2009, pp. 129-141.

[29] German, B. J. and Sivakumar, H., "Laplacian Equivalents to Subsonic Cascade Flows," 47th AIAA Aerospace Sciences Meeting Including The New Horizons Forum and Aerospace Exposition, AIAA 2009-927, 2009.

[30] Franke, R., "Scattered Data Interpolation: Tests of Some Methods," Mathematics of Computation, Vol. 38, 1982, pp. 181-200.

[31] Kansa, E. J., "Multiquadrics: A Scattered Data Approximation Scheme with Applications to Computational Fluid Dynamics I," Computers and Mathematics with Applications, Vol. 19, No. 8/9, 1990, pp. 127-145.

[32] Kansa, E. J., "Multiquadrics: A Scattered Data Approximation Scheme with Applications to Computational Fluid Dynamics II," Computers and Mathematics with Applications, Vol. 19, 1990, pp. 147-161. 
[33] Carlson, R. E. and Foley, T. A., "The Parameter R2 in Multiquadric Interpolation," Computers and Mathematics with Applications, Vol. 21, 1991, pp. 29-42.

[34] Kansa, E. J. and Carlson, R. E., "Improved Accuracy of Multiquadric Interpolation Using Variable Shape Parameters," Computers and Mathematics with Applications, Vol. 24, 1992, pp. 99-120.

[35] Golberg, M. A., Chen, C. S., and Karur, S. R., "Improved Multiquadric Approximation for Partial Differential Equations," Engineering Analysis with Boundary Elements, Vol. 18, 1996, pp. 9-17.

[36] Rippa, S., "An Algorithm for Selecting a Good Value for the Parameter c in Radial Basis Function Interpolation," Advances in Computational Mathematics, Vol. 11, 1999, pp. 193-210.

[37] Wang, J. G. and Liu, G. R., "On the Optimal Shape Parameters of Radial Basis Functions Used for 2-D Meshless Methods," Computer Methods in Applied Mechanics and Engineering, Vol. 191, 2002, pp. 2611-2630.

[38] Fornberg, B. and Wright, G., "Stable Computation of Multiquadric Interpolants for All Values of the Shape Parameter," Computers and Mathematics with Applications, Vol. 48, 2004, pp. 853-867. 
[39] Wertz, J., Kansa, E. J., and Ling, L., "The Role of the Multiquadric Shape Parameters in Solving Elliptic Partial Differential Equations," Computers and Mathematics with Applications, Vol. 51, 2006, pp. 1335-1348.

[40] Fasshauer, G. E. and Zhang, J. G., "On Choosing the "Optimal" Shape Parameters for RBF approximation," Numerical Algorithms, Vol. 45, 2007, pp. 345-368.

[41] Lock, R. C., "Test Cases for Numerical Methods in Two-Dimensional Transonic Flows," Tech. Rep. 575, AGARD, Neuilly-sur-Siene, France, 1970.

[42] Liu, G. R., Meshfree Methods: Moving Beyond the Finite Element Method, Taylor and Francis Group, Boca Raton, FL, 2nd ed., 2010.

[43] Nguyen, V. P., Rabczuk, T., Bordas, S., and Duflot, M., "Meshless Methods: A Review and Computer Implementation Aspects," Mathematics and Computers in Simulation, Vol. 79, 2008, pp. 763-813.

[44] Forsythe, G. E. and Wasow, W. R., Finite Difference Methods for Partial Differential Equations, John Wiley and Sons, 1960.

[45] Batina, J. T., "A Gridless Euler/Navier-Stokes Solution Algorithm for Complex Two-Dimensional Applications," Tech. rep., NASA, TM-107631, 1992.

[46] Oñate, E., Idelssohn, S., Zienkiewicz, O. C., and Taylor, R. L., "A Finite Point Method in Computational Mechanics: Applications to Convective Transport 
and Fluid Flow," International Journal for Numerical Methods in Engineering, Vol. 39, 1996, pp. 3839-3866.

[47] Chung, K. C., "A Generalized Finite-Difference Method for Heat Transfer Problems of Irregular Geometries," Numerical Heat Transfer, Vol. 4, 1981, pp. 345357.

[48] Shu, C., Ding, H., and S., Y. K., "Local Radial Basis Function-Based Differential Quadrature Method and its Application to Solve Two-Dimensional Incompressible Navier-Stokes Equations," Computer Methods in Applied Mechanics and Engineering, Vol. 192, 2003, pp. 941-954.

[49] Liszka, T. and Orkisz, J., "The Finite Difference Method at Arbitrary Irregular Grids and its Application in Applied Mechanics," Computers and Structures, Vol. 11, 1980, pp. 83-95.

[50] MacNeal, R. H., "An Asymmetrical Finite Difference Network," Quarterly of Applied Mathematics, Vol. 11, 1953, pp. 295-310.

[51] Liszka, T., "An Interpolation Method for an Irregular Net of Nodes," International Journal for Numerical Methods in Engineering, Vol. 20, 1984, pp. 15991612.

[52] Batina, J. T., "A Gridless Euler/Navier-Stokes Solution Algorithm for ComplexAircraft Applications," Tech. rep., NASA, TM-107727, 1993. 
[53] Oñate, E., Idelssohn, S., Zienkiewicz, O. C., Taylor, R. L., and Sacco, C., "A Stabilized Finite Point Method for Analysis of Fluid Mechanics Problems," Computer Methods in Applied Mechanics and Engineering, Vol. 139, 1996, pp. 315346.

[54] Oñate, E. and Idelssohn, S., "A Mesh-Free Finite Point Method for AdvectiveDiffusive Transport and Fluid Flow Problems," Computational Mechanics, Vol. 21, 1998, pp. 283-292.

[55] Oñate, E., Sacco, C., and Idelssohn, S., "Meshless Analysis fo Incompressible Flows Using the Finite Point Method," Technical report, International Center for Numerical Methods in Engineering (CIMNE), Barcelona, Spain, 2000.

[56] Löhner, R., Sacco, C., Oñate, E., and Idelssohn, S., "A Finite Point Method for Compressible Flow," International Journal for Numerical Methods in Engineering, Vol. 53, 2002, pp. 1765-1779.

[57] Sridar, D. and Balakrishnan, N., "An Upwind Finite Difference Scheme for Meshless Solvers," Journal of Computational Physics, Vol. 189, 2003, pp. 1-29.

[58] Sridar, D. and Balakrishnan, N., "Convergence Acceleration of an Upwind Least Squares Finite Difference Based Meshless Solver," AIAA Journal, Vol. 44, No. 10, 2006, pp. 2189-2196. 
[59] Morinishi, K., "Effective Accuracy and Conservation Consistency of Gridless Type Solver," Computational Fluid Dynamics 2000: Proceedings of the First International Conference on Computational Fluid Dynamics, 2000.

[60] Löhner, R. and Oñate, E., "An Advancing Front Point Generation Technique," Communications in Numerical Methods in Engineering, Vol. 14, 1998, pp. 10971108.

[61] Löhner, R. and Oñate, E., "A General Advancing Front Technique for Filling Space with Arbitrary Objects," International Journal for Numerical Methods in Engineering, Vol. 61, 2004, pp. 1977-1991.

[62] Katz, A. and Jameson, A., "A Comparison of Various Meshless Schemes Within a Unified Algorithm," 47th AIAA Aerospace Sciences Meeting, AIAA 2009-596, 2009.

[63] Chinchaptnam, P. P., Djidjeli, K., and Nair, P. B., "Meshless RBF Collocation for Steady Incompressible Viscous Flows," 36th AIAA Fluid Dynamics Conference, AIAA 2006-3525, 2006.

[64] McDonald, R. A. and Ramos, A., "Hermite TLS for Unstructured and Mesh-free Derivative Estimation Near and On Boundaries," Submitted to the 2011 AIAA Aerospace Sciences Meeting, 2011. 
[65] McLeod, R. J. Y. and Baart, M. L., Geometry and Interpolation of Curves and Surfaces, Cambridge University Press, 1998.

[66] Kinney, D. J., Finite Element Solution of the Full Potential Equation Over Aircraft Configurations Using Unstructured Tetrahedral Grids, Ph.d. dissertation, University of California Davis, 1994.

[67] ASME, Journal of Fluids Engineering Editorial Statement on the Control of Numerical Accuracy.

[68] AIAA, Editorial Policy Statement on Numerical and Experimental Accuracy.

[69] Zingg, D. W., "Grid Studies for Thin-Layer Navier-Stokes Computations of Airfoil Flowfields," 30th AIAA Aerospace Sciences Meeting and Exhibit, AIAA 92$0184,1992$.

[70] Katz, A. J., Meshless Methods for Computational Fluid Dynamics, Ph.D. thesis, Stanford University, 2009.

[71] Carolina, L., Tsai, H. M., and Liu, F., "An Embedded Cartesian Grid Euler Solver with Radial Basis Function for Boundary Condition Implementation," 46th AIAA Aerospace Sciences Meeting, AIAA 2008-532, 2008.

[72] Luo, H., Baum, J. D., and Lohner, R., "A Hybrid Cartesian Grid and Gridless Method for Compressible Flows," 43rd AIAA Aerospace Sciences Meeting, AIAA 2005-492, 2005. 
[73] Luo, H., Baum, J. D., and Lohner, R., "A Hybrid Building-Block and Gridless Method for Compressible Flows," 36th AIAA Fluid Dynamics Conference and Exhibit, AIAA 2006-3710, 2006.

[74] Johnson, F. T., Samant, S. S., Bieterman, M. B., Melvin, R. G., Young, D. P., Bussoletti, J. E., and Hilmes, C. L., "TranAir: A Full-Potential SolutionAdaptive, Rectangular Grid Code for Predicting Subsonic, Transonic, and Supersonic Flows About Arbitrary Configurations," Tech. rep., NASA, CR 4348, 1992.

[75] Ashby, D. L., "Potential Flow Theory and Operation Guide for the Panel Code PMARC14," Tech. rep., NASA, TM-1999-209582, 1999. 


\section{Appendix A}

\section{Influence Coefficients}

The numerical integration of the elements within the $[H],[G],\left[H_{u}\right],\left[H_{v}\right],\left[G_{u}\right]$, and $\left[G_{v}\right]$ matrices will be discussed in this appendix. All of these integrals are related to the constant strength doublet and source potentials described in Katz and Plotkin [8] and are reproduced here with the nomenclature that applies to this thesis. Figure A.1 is reproduced from Katz and Plotkin in order to depict the different variables that will be used in this appendix.

\section{A.1 $[H]$ Matrix}

Recall that the elements within the $[H]$ matrix are composed of integrating the normal flux of the fundamental solution along each of the elements comprising the geometry surface.

$$
H_{i j}=\int_{S_{j}} q_{i j}^{*} d S_{j}
$$

Each of these integrals is analogous to the calculation of the potential induced by a constant strength doublet. Before the integral can be analytically evaluated the 


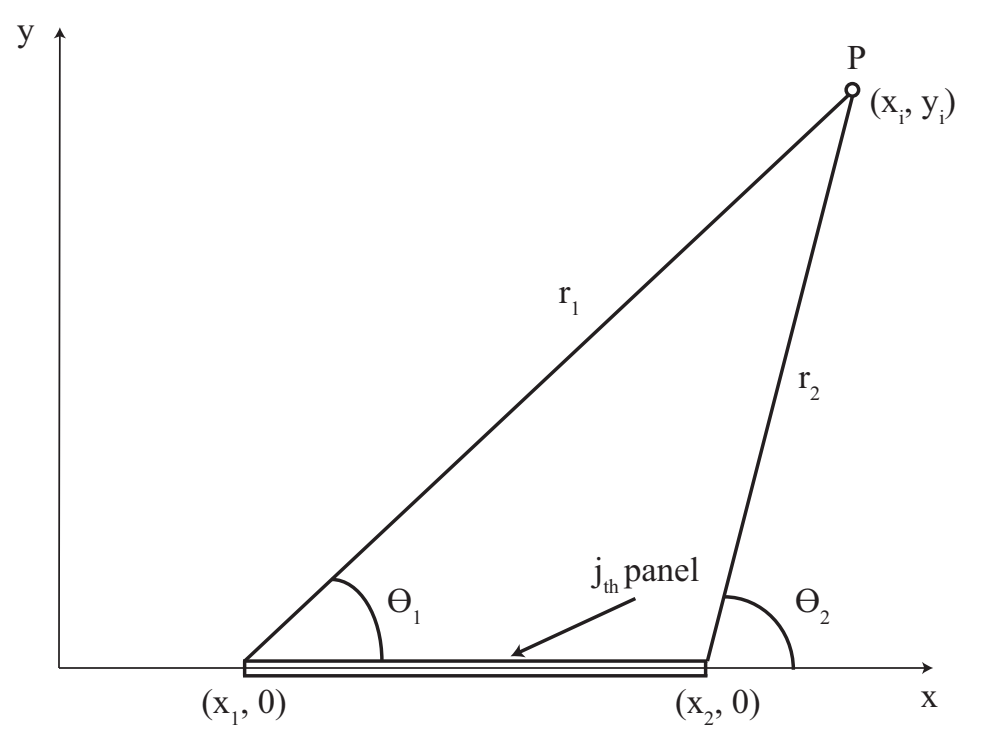

Figure A.1: Panel Nomenclature

coordinates of the panel endpoints and the collocation point need to be transformed into the $j_{\text {th }}$ panels local coordinate system. Once this transformation is performed the integral can then be easily evaluated.

$$
\int_{S_{j}} q_{i j}^{*} d S_{j}=-\frac{1}{2 \pi} \int_{x_{1}}^{x_{2}} \frac{y_{i}}{\left(x_{i}-x_{0}\right)^{2}+y_{i}^{2}} d x_{0}
$$

This integral can be expressed in terms of the angles shown in A.1. When $i=j$ the point $P$ is located on the middle of the panel. Special consideration needs to be taken for this case which results in the integral reducing to $\frac{1}{2}$. Since the potential is a scalar function, there is no need for transforming back into global coordinates.

$$
H_{i j}=\left\{\begin{array}{r}
-\frac{1}{2 \pi}\left(\theta_{2}-\theta_{1}\right) \mathrm{i} \neq \mathrm{j} \\
\frac{1}{2} \mathrm{i}=\mathrm{j}
\end{array}\right.
$$




\section{A.2 $[G]$ Matrix}

Recall that the elements within the $[G]$ matrix are composed of integrating the fundamental solution along each of the elements comprising the geometry surface.

$$
G_{i j}=\int_{S_{j}} u_{i j}^{*} d S_{j}
$$

Each of these integrals is analagous to the calculation of the potential induced by a constant strength source. Once the same transformation discussed in the previous section is performed, the integral can be analytically evaluated.

$$
\int_{S_{j}} u_{i j}^{*} d S_{j}=-\frac{1}{2 \pi} \int_{x_{1}}^{x_{2}} \ln \sqrt{\left(x_{i}-x_{0}\right)^{2}+y_{i}^{2}}, d x_{0}
$$

This integral can be expressed in terms of the distances and angles shown in A.1. Once again special considerations need to be taken when $i=j$.

$$
G_{i j}=\left\{\begin{array}{r}
-\frac{1}{4 \pi}\left[\left(x_{i}-x_{1}\right) \ln r_{1}^{2}-\left(x_{i}-x_{2}\right) \ln r_{2}^{2}+2 y_{i}\left(\theta_{2}-\theta_{1}\right)\right] \mathrm{i} \neq \mathrm{j} \\
-\frac{1}{4 \pi}\left(x_{2}-x_{1}\right) \ln \left(\frac{x_{2}-x_{1}}{2}\right)^{2} \mathrm{i}=\mathrm{j}
\end{array}\right.
$$

\section{A.3 $\left[\bar{H}_{u}\right]$ and $\left[\bar{H}_{v}\right]$ Matrices}

The $\left[\bar{H}_{u}\right]$ and $\left[\bar{H}_{v}\right]$ matrices are the derivatives of the $[\bar{H}]$ matrix in the $\mathrm{x}$ and $\mathrm{y}$ directions respectively. Therefore the elements within these matrices are simply the $\mathrm{x}$ and $\mathrm{y}$ derivatives of the integrals that comprise the elements of $[\bar{H}]$.

Recall that the elements within the $\left[\bar{H}_{u}\right]$ and $\left[\bar{H}_{v}\right]$ matrices have the form:

$$
\bar{H}_{u i j}=\int_{S_{j}} \frac{\partial q_{i j}^{*}}{\partial x} d S_{j}
$$




$$
\bar{H}_{v i j}=\int_{S_{j}} \frac{\partial q_{i j}^{*}}{\partial y} d S_{j}
$$

These integrals correspond to taking the $\mathrm{x}$ and $\mathrm{y}$ derivatives of (A.2) respectively. They also correspond to calculating the horizontal and vertical velocities, in local panel coordinates, induced by a constant strength doublet.

$$
\begin{aligned}
\int_{S_{j}} \frac{\partial q_{i j}^{*}}{\partial x} d S_{j} & =\frac{1}{2 \pi} \int_{x_{1}}^{x_{2}} \frac{\left(x_{i}-x_{0}\right) y_{i}}{\left[\left(x_{i}-x_{0}\right)^{2}+y_{i}^{2}\right]^{2}} d x_{0} \\
\int_{S_{j}} \frac{\partial q_{i j}^{*}}{\partial y} d S_{j} & =\frac{1}{2 \pi} \int_{x_{1}}^{x_{2}} \frac{\left(x_{i}-x_{0}\right)^{2}-y_{i}^{2}}{\left[\left(x_{i}-x_{0}\right)^{2}+y_{i}^{2}\right]^{2}} d x_{0}
\end{aligned}
$$

As in the previous sections, this integral can be analytically integrated. Since the $\left[\bar{H}_{u}\right]$ and $\left[\bar{H}_{v}\right]$ matrices are only applied to points not located on the boundary, no special conditions for $i=j$ will be addressed here. It is also important to note that the velocity is a vector valued function, therefore the velocities calculated in local panel coordinates need to be transformed back into global coordinates.

$$
\begin{aligned}
& \bar{H}_{u i j}=\frac{1}{2 \pi}\left[\frac{y_{i}}{\left(x_{i}-x_{1}\right)^{2}+y_{i}^{2}}-\frac{y_{i}}{\left(x_{i}-x_{2}\right)^{2}+y_{i}^{2}}\right] \\
& \bar{H}_{v i j}=\frac{1}{2 \pi}\left[\frac{x_{i}-x_{1}}{\left(x_{i}-x_{1}\right)^{2}+y_{i}^{2}}-\frac{x_{i}-x_{2}}{\left(x_{i}-x_{2}\right)^{2}+y_{i}^{2}}\right]
\end{aligned}
$$

\section{A.4 $\left[G_{u}\right]$ and $\left[G_{v}\right]$ Matrices}

The $\left[G_{u}\right]$ and $\left[G_{v}\right]$ matrices are the derivatives of the $[G]$ matrix in the $\mathrm{x}$ and $\mathrm{y}$ directions respectively. Therefore the elements within these matrices are simply the $\mathrm{x}$ and $\mathrm{y}$ derivatives of the integrals that comprise the elements of $[G]$. Recall that the 
elements within the $\left[G_{u}\right]$ and $\left[G_{v}\right]$ matrices have the form:

$$
\begin{aligned}
G_{u i j} & =\int_{S_{j}} \frac{\partial u_{i j}^{*}}{\partial x} d S_{j} \\
G_{v i j} & =\int_{S_{j}} \frac{\partial u_{i j}^{*}}{\partial y} d S_{j}
\end{aligned}
$$

These integrals correspond to taking the $\mathrm{x}$ and $\mathrm{y}$ derivatives of (A.5) respectively. They also correspond to calculating the horizontal and vertical velocities, in local panel coordinates, induced by a constant strength source.

$$
\begin{gathered}
\int_{S_{j}} \frac{\partial u_{i j}^{*}}{\partial x} d S_{j}=\frac{1}{2 \pi} \int_{x_{1}}^{x_{2}} \frac{x_{i}-x_{0}}{\left(x_{i}-x_{0}\right)^{2}+y_{i}^{2}} d x_{0} \\
\int_{S_{j}} \frac{\partial u_{i j}^{*}}{\partial y} d S_{j}=\frac{1}{2 \pi} \int_{x_{1}}^{x_{2}} \frac{y_{i}}{\left(x_{i}-x_{0}\right)^{2}+y_{i}^{2}} d x_{0}
\end{gathered}
$$

As with the $\left[\bar{H}_{u}\right]$ and $\left[\bar{H}_{v}\right]$ matrices, the $\left[G_{u}\right]$ and $\left[G_{v}\right]$ matrices are only applied to points not located on the boundary. Also the velocities calculated in local panel coordinates need to be transformed back into global coordinates.

$$
\begin{gathered}
G_{u i j}=\frac{1}{2 \pi} \ln \frac{r_{1}}{r_{2}} \\
G_{v i j}=\frac{1}{2 \pi}\left(\theta_{2}-\theta_{1}\right)
\end{gathered}
$$

